\title{
Helicopter Impulsive Noise: Theoretical and Experimental Status
}

\section{F.H. Schmitz and Y.H. Yu}

November 1983

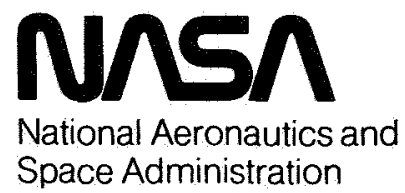

United States Army

Aviation Research and Development Command 


\section{Helicopter Impulsive Noise: Theoretical and Experimental Status}

F. H. Schmitz,

Y. H. Yu, Aeromechanics Laboratory, Research and Technology Laboratories

U. S. Army Aviation Research and Development Command

Ames Research Center, Moffett Field, CA 


\section{ABSTRACT}

The theoretical and experimental status of helicopter impulsive noise is reviewed. The two major source mechanisms of helicopter impulsive noise are addressed: high-speed Impulsive noise and blade-vortex interaction impulsive noise. A thorough physical explanation of both generating mechanisms is presented together with model and full-scale measurements of the phenomena. Current theoretical prediction methods are compared with experimental findings of isolated rotor tests. The noise generating mechanisms of high speed impulsive noise are fairly we11 understood - theory and experiment compare nicely over Mach number ranges typical of today's helicopters. For the case of blade-vortex interaction noise, understanding of noise generating mechanisms and theoretical comparison with experiment are less satisfactory. Several methods for improving theory-experiment are suggested. 


\section{INTRODUCTION}

Over the past 15 years, many researchers have devoted a significant portion of their lives attempting to understand the basic generating mechanisms of sound produced by rotors. In many instances, these efforts have been quite successful, with each new advance bringing the goals of understanding, modeling, and acoustic prediction of rotor noise much closer. Obviously, this rosy outlook is not true in every instance, and there are areas in which much work is needed. However, as it is with the squeaky whee1, the noises that are loudest - the more important external noise sources - are getting the most attention. For helicopters, these loudest and often the most annoying sounds are impulsive in nature, originating on both main and tail rotors.

The threat (and follow-through) of noise rules for helicopters is having a beneficial effect on all acoustic aspects of rotors. In many cases, noise is now treated seriously early in the design process instead of as a problem to be fixed during production. However, there still remains the important trade-off of noise and performance. If reducing noise increases performance, the likelihood of an "acoustic design change" is enhanced. However, if the acoustic change decreases vehicle performance, the change must be weighed against other competing factors. If the acoustician is unsure of his design guidelines, his position in the design group is seriously weakened, and, in general, he will have less influence in the entire design process. Thus, it is imperative that the major sources of noise be addressed even at the expense of perhaps more interesting research phenomena.

Complicating this entire picture is the degree to which the vehicle itself enters into the noise-generation process. In the case of the helicopter, almost the entire aerodynamic noise-radiation process is tied to the aerodynamic state of the main and tail rotors. The disciplines of aerodynamics, rotor-body dynamics, and rotor-blade dynamics can all influence the exact state of the rotor. Theoretically, the problem is almost intractable. There are so many possible factors that can influence the radiated noise that some experimental guidance is necessary to sort out important controlling parameters.

Because there are so many sources of noise on a helicopter and because it would take a much larger document than this monograph to review adequately the status of all of them, we will not attempt it. Instead, we will concentrate on a status report on helicopter impulsive noise which includes high-speed (HS) impulsive as well as blade-vortex interaction (BVI) impulsive noise. The reader is asked to consult references 1-3 for a more general review of the status of helicopter noise. In particular, references 2 and 3, respectively, give excellent theoretical and experimental reviews. Limiting this effort to the impulsive noise of helicopters is not as restrictive as it first seems, for these noise sources have received and probably will continue to receive most of the research attention, probably because they are responsible for much of the radiated noise on today's helicopters. 
Most of what we shall review can be mathematically represented by the following general, we11-known integral equation governing the noise that is radiated from a body in arbitrary motion:

$$
\begin{aligned}
& 4 \pi a_{o^{2}}^{2-1}(\vec{x}, t)=\frac{\partial^{2}}{\partial x_{i} \partial x_{j}} \iint\left[\frac{T_{i j}}{r\left|1-M_{r}\right|}\right]_{\tau} d V(\vec{n}) \\
& -\frac{\partial}{\partial x_{i}} \int\left[\frac{P_{i j}{ }^{n} j}{\left.r \mid 1-M_{r}\right\rceil}\right]_{\tau} d s(\hat{n}) \\
& +\frac{\partial}{\partial t} \iint\left[\frac{\rho_{0} v_{n}}{\left.r \mid 1-M_{r}\right\rceil}\right]_{\tau} d S(\hat{n})
\end{aligned}
$$

where

$$
T_{i j}=\rho u_{i} u_{j}+P_{i j}-a_{o}^{2} \rho^{i} \delta_{i j}
$$

This equation was derived in reference 4 and expanded upon by many researchers (e.g., refs. 5-7). Far-field acoustic pressure is explicitly expressed in terms of integrals over the blade surface and the surrounding volume in a reference frame moving with the blade surface.

We shall begin by looking at some experimental results to see if we can correlate specific terms in the theory (eq. (1)) to specific features of the radiated noise. Before we delve into quantitative comparisons, it is helpful to look at helicopter deterministic noise sources qualitatively. Fortunately, the loudest and most annoying sounds are often the easiest to relate to aerodynamic events on the blade. Therefore, we sha11 begin with a physical explanation of impulsive noises of the helicopter. These arguments will then be used to explain many of the measured characteristics of helicopter impulsive noise. Finally, we shall compare theory with measured data and explain what additional effects are needed to model the resulting acoustic radiation.

Much of the work presented here has borrowed from our research and from that of colleagues at the U.S. Army Aeromechanics Laboratory, Research and Technology Laboratories (AVRADCOM), over the past 10 years. We have tried to use the research of others to complement and enhance our interpretation of the present status of helicopter impulsive noise. Because our point of view is one of an involved researcher, there are undoubtedly some unintentional biases in the monograph for which we offer our apologies. 
It is possible to analyze measured acoustic data in the frequency or time domains. However, in instances when we are dealing with helicopter impulsive noise, it is much easier to diagnose the cause of that radiated sound if a time-domain analysis is used. The sequence of events that cause the radiated noise are all related to events that happen as the rotor rotates (changes azimuth angle $\Psi$ ). Thus, it is natural to think of $\Psi$ as a "source time" which tends to order the events an observer may hear.

For simplicity in these first arguments, we shall only consider terms on the right-hand side of equation (1) which can be derived by linearizing the governing mass and momentum equations. Therefore, only the last two terms of equation (1) are discussed. It turns out that the last integral governs "linear" high-speed impulsive noise, and the second terms is a large contributor to blade-vortex interaction impulsive noise.

\section{Linear-Thickness Impulsive Noise}

"Linear-thickness noise" can be represented by considering equation (1) with only the third term appearing on the right-hand side. The resulting simplified integral equation becomes

$$
P^{\prime}(\vec{x}, t)=\frac{1}{4 \pi} \frac{\partial}{\partial t} \iint\left[\frac{\rho_{0} v_{n}}{r\left|1-M_{r}\right|}\right]_{\tau} d S(\vec{n})
$$

This equation states that the acoustic pressure is really the sum of a distribution of singularities over the blade surface. It is important to remember that sound generated by each singularity must travel a slightly different path to the observer's location and, therefore, will arrive at a different observer time $t$. The simplest way of describing this integration is to divide the tip section of the blade into two chordwise panels. (We only treat the blade-tip region because we know that it is the source of most of the acoustic radiation.) The first panel is composed of "sources" and the second of "sinks," as shown in figure 1 for a single-bladed rotor.

One of the most interesting aspects of the evaluation of the thickness integra1

$$
\iint\left[\frac{\rho_{0} v_{n}}{r\left|1-M_{r}\right|}\right]_{\tau} d S(\vec{n})
$$

is that the integration is a function of $1 /\left(r\left|1-M_{r}\right|\right)$ which depends on the observer's location. The factor $1 /\left(1-M_{r}\right)$ represents the Doppler amplification of acoustic signals and is a strong function of the Mach 
OUTER SECTION

OF THE BLADE
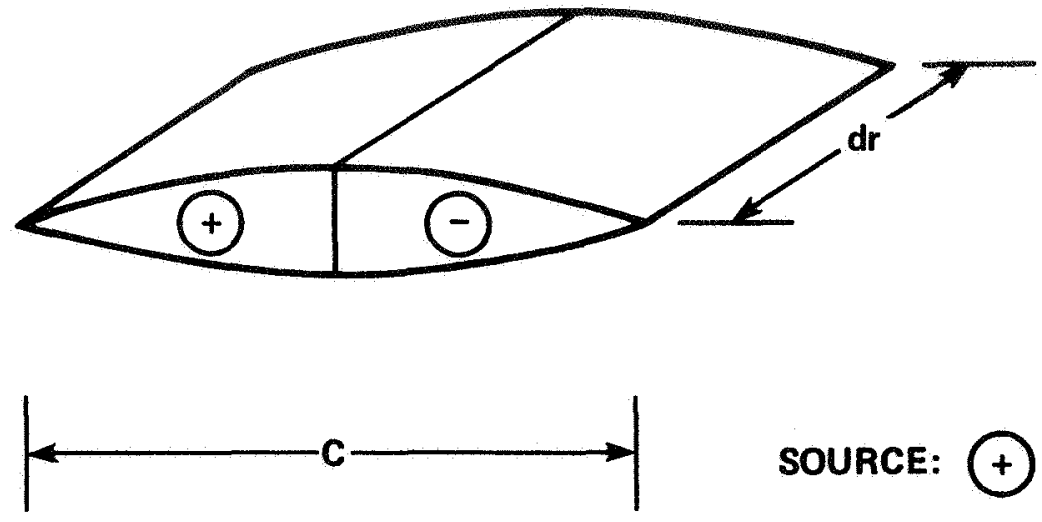

SOURCE:

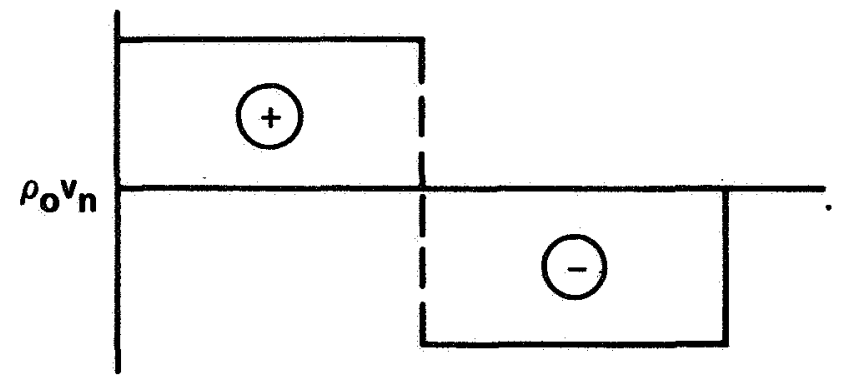

SINK:

Figure 1.- Acoustic sources and sinks.

number in the radiation direction, $M_{r}$. As shown in figure 2, for an observer in the disk plane located directly ahead of the rotor's flight path, $M_{r}$ becomes a maximum when $\psi \approx 90^{\circ}$. Thus, we would expect the thickness noise peak to originate near $\psi=90^{\circ}$.

Now let us sketch a graphical outline of the integration of the integral for the in-plane observer located directly ahead of the rotor (fig. 3). First, consider the simple source $\left(\rho_{o} v_{n}=q_{t}\right)$. Then

$$
\iint\left[\frac{q_{t}}{r\left|1-M_{r}\right|}\right] d s(\vec{r})
$$

becomes as in figure 3. Similarly, the integral of the simple "sink" becomes the same curve shifted (delayed) in time $c / 2(\Omega R) \mathrm{sec}$

$$
t_{\text {source }} \approx t_{\text {sink }}+\frac{c \sin \psi}{2(\Omega R)}
$$




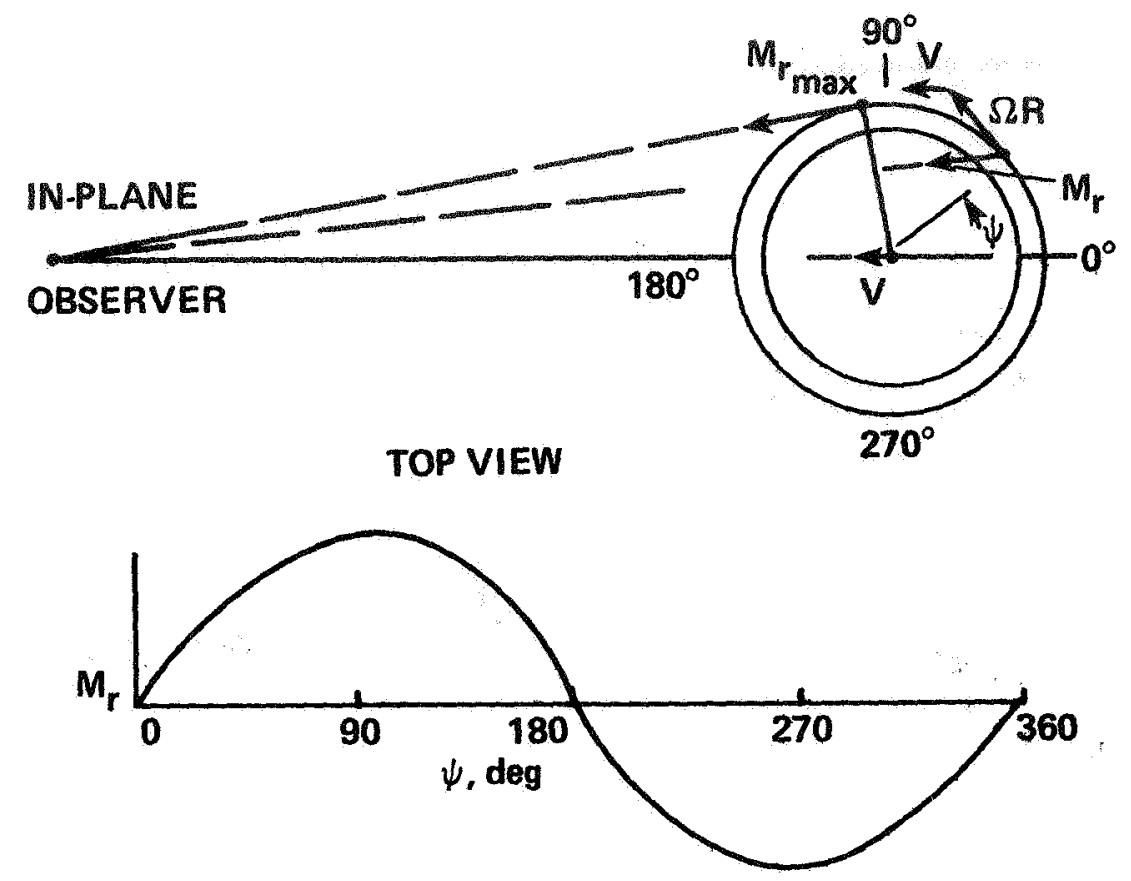

Figure 2.- Variation of $M_{r}$ over azimuthal angles.

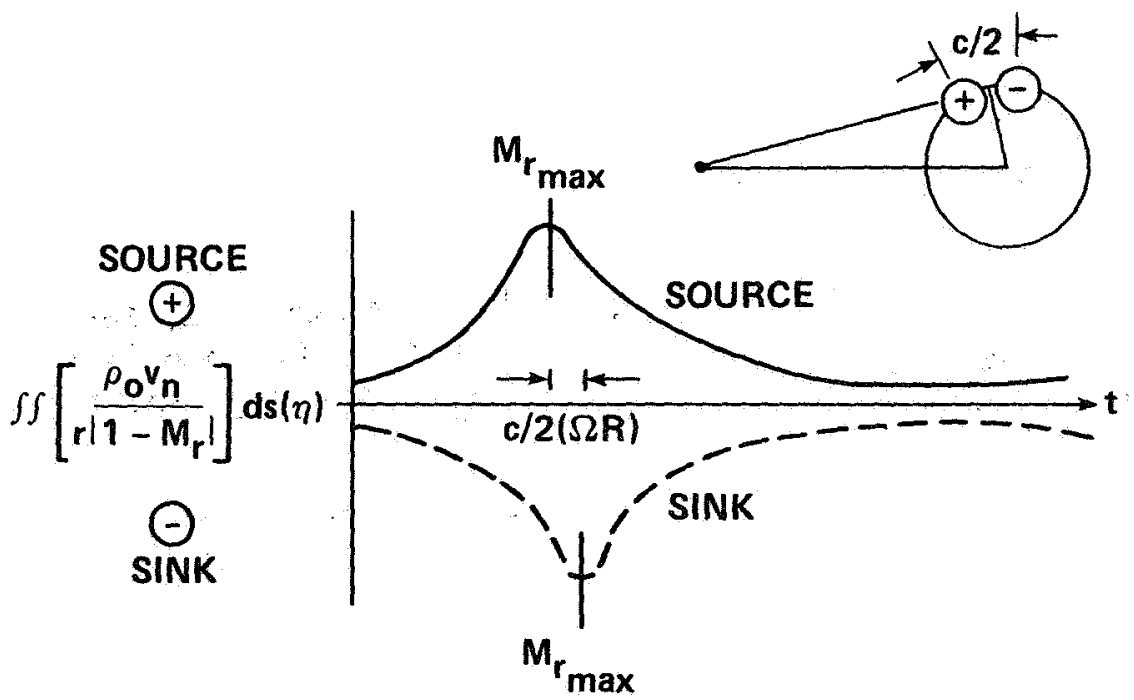

Figure 3.- Variation of $M_{r}$ over observer time.

Although not exp1icitly shown, a11 of the above arguments depend on the correct evaluation of the retarded time-equation, $\tau=t-r / a_{0}$. The simple shift in observer time causes the two sources to not cancel. This is the major mechanism of 1inear-thickness noise. It is characterized by a large negative-pressure pulse; a similar set of arguments was presented in reference 8 (see fig. 4). 

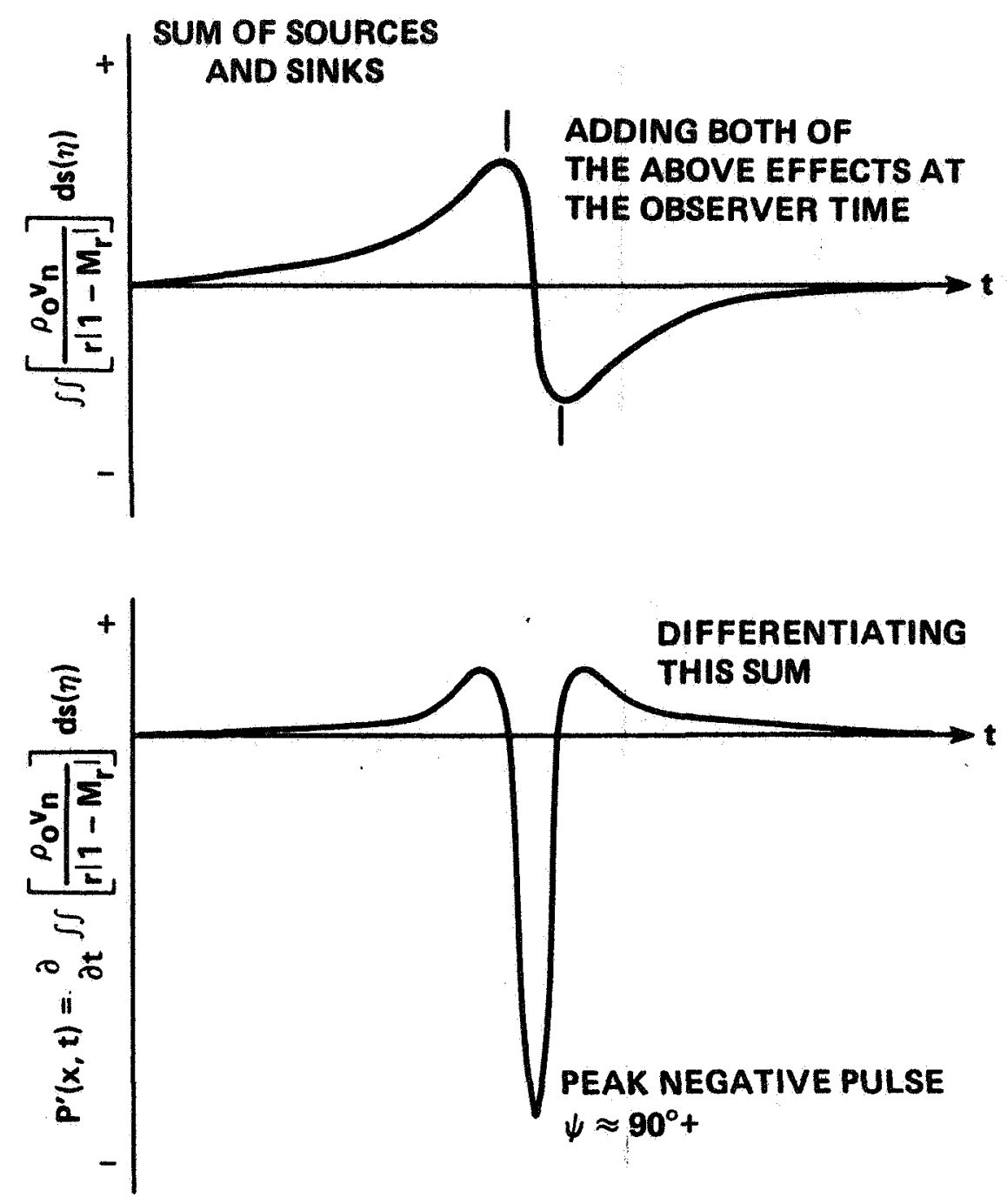

Figure 4.- Summation of sources and sinks for given observer time and its derivative. 
Consider the same integral for an observer situated directly beneath the rotor's axis. In this location, the observer sees no difference in time or in attitude for the source and sink integration. Thus, when both contributions are summed and the time-derivative taken, little radiation is found. The contributions from the simple source and sink cancel, causing no acoustic radiation. These simple arguments explain why the linear-thickness noise only radiates near the plane of the rotor disk. This will be confirmed when we look at some experimental data.

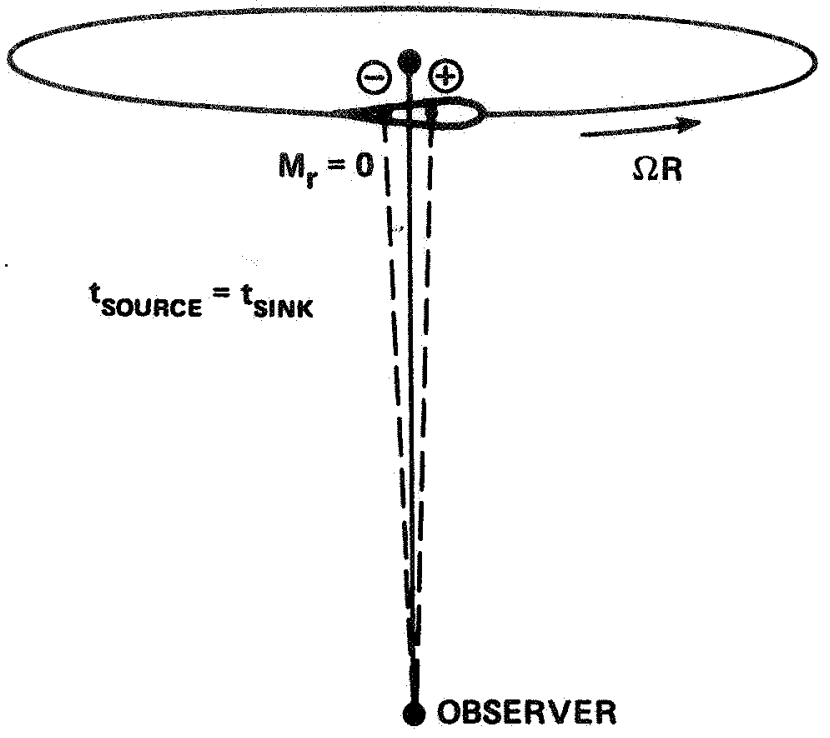

Blade-Vortex Interaction Impulsive Noise

The qualitative characteristics of blade-vortex interaction (BVI) noise can be deduced in a similar manner. Again, we sha11 perform our analysis in the time-domain so that we may order the acoustic events in $\psi$ and finally in observer time $t$. Consider the top view of a twobladed helicopter rotor at an advance ratio of $0.145(\mu=V / \Omega R=0.145)$ which is shown in figure 5. The epicycloid-like patterns were derived from a "free-wake" computer code. However, it is known from experience that the top view of both the rigid and free wakes is quite similar, suggesting that the simple rigid-wake tip vortex epicycloid pattern is a good first-order representation of the tip-vortex patterns of the free-wake analysis.

We know from theoretical considerations that most of the radiated noise is generated near the rotor tip. We also would expect blade-vortex interaction noise to occur when the rotor blade (outer 20-30\%) passes close to the trailing-tip vortices. As seen from figure 5, there are seven possible blade-vortex interactions (1abeled 1-7).

The strength of each interaction is governed by

1. Local strength of the tip vortex

2. Core size of the tip vortex

3. Local interaction angle of the blade and the vortex line

4. Vertical separation between the vortex and the blade 


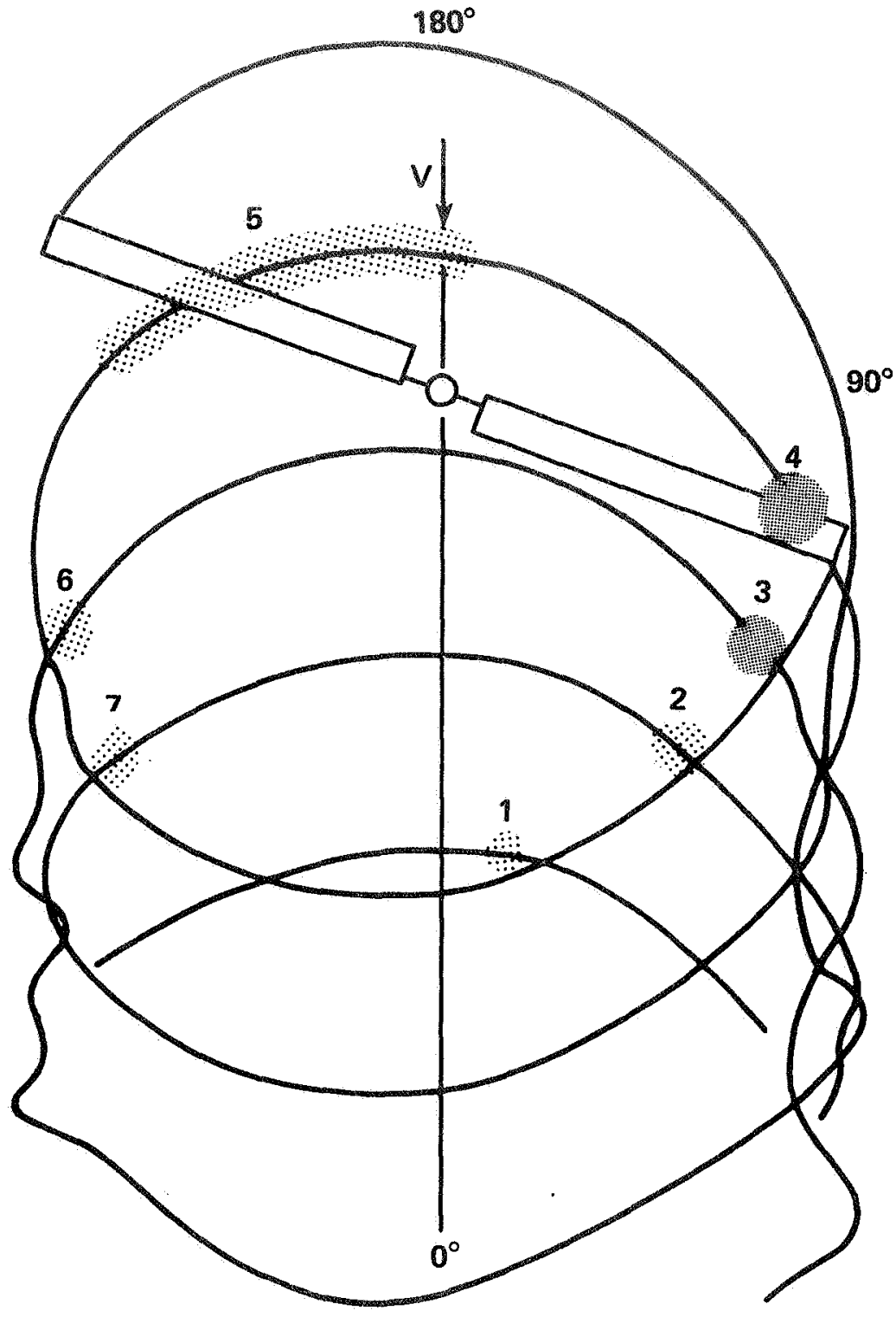

Figure 5.- Blade-vortex intersections during partial-power descent (from ref. 9).

In general, the induced velocity of the rotor disk tends to make all of the tip vortices pass under the rotor disk in level steady-state flight conditions for $\mu \approx 0.15$. However, if the rotor operates in steady descending flight, then the positive inflow (upflow) tends to force the epicycloid-type pattern into the rotor disk plane, causing strong bladevortex interactions (see fig. 6).

The net result of such considerations is shown in figure 7 . Of these potential seven blade-vortex interaction (BVI) encounters, only a few are known to radiate strong impulsive noises. Consider encounters numbered 1-4, which are all on the advancing side of the rotor disk. 


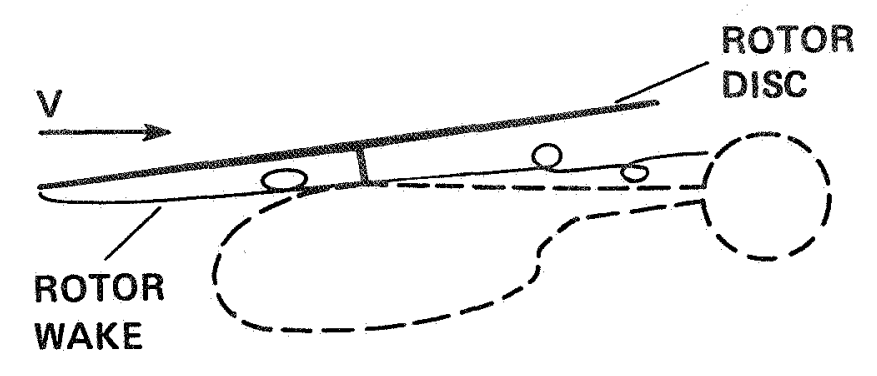

LEVEL FLIGHT

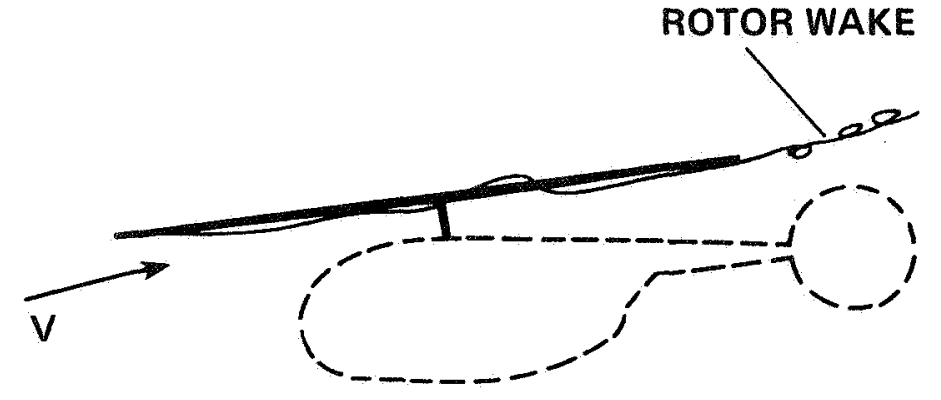

DESCENDING FLIGHT

Figure 6.- Interaction of tip vortices with a rotor disk for level and descending flights.

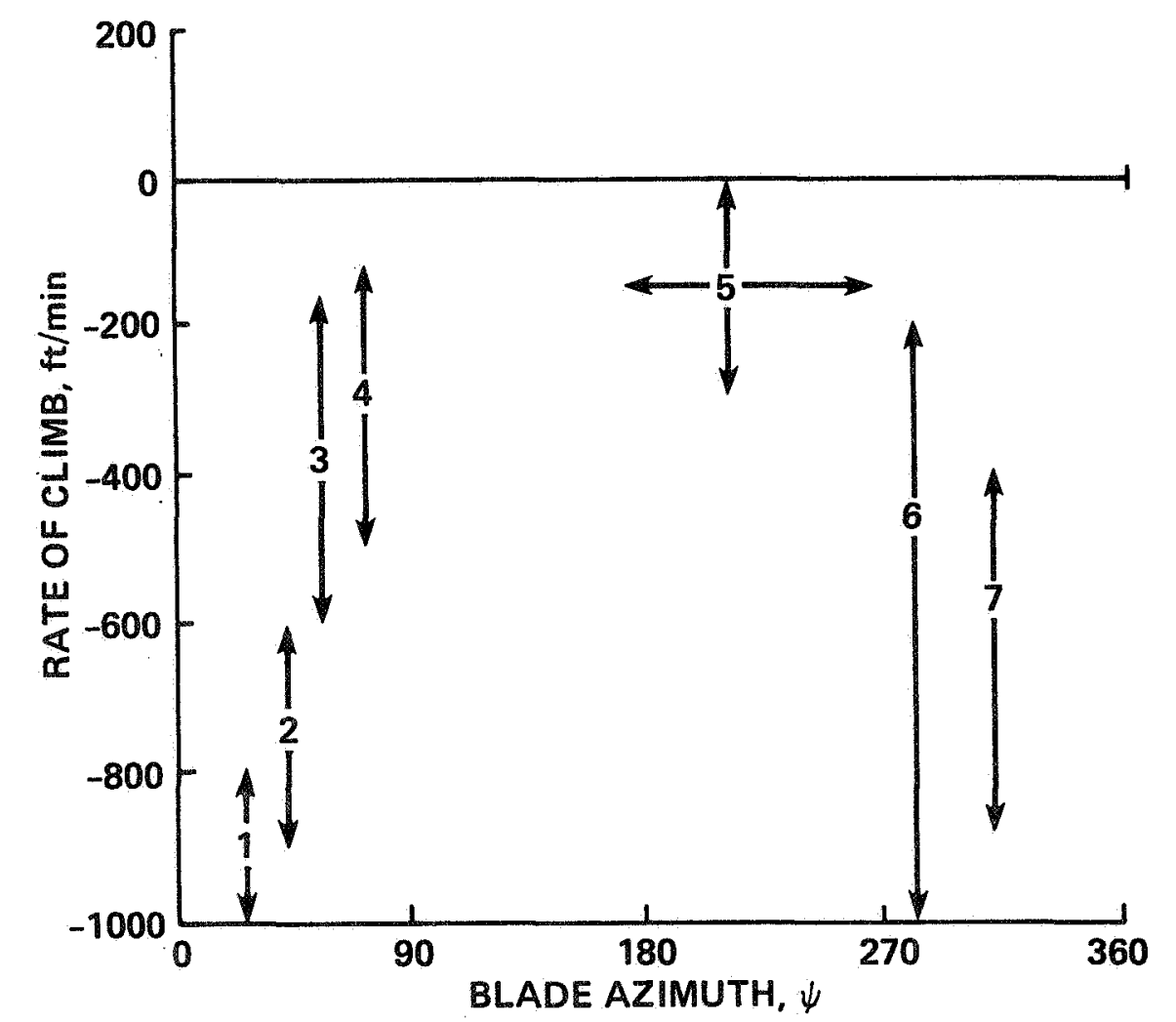

Figure 7.- Flight conditions for potential blade-vortex interactions. 
Number 3 in particular is an encounter in which the blade and the vortex are almost parallel. In this case, we can use some simple two-dimensional arguments to estimate the correct shape of such a pulse. An advancing blade might see the following (see fig. 8):
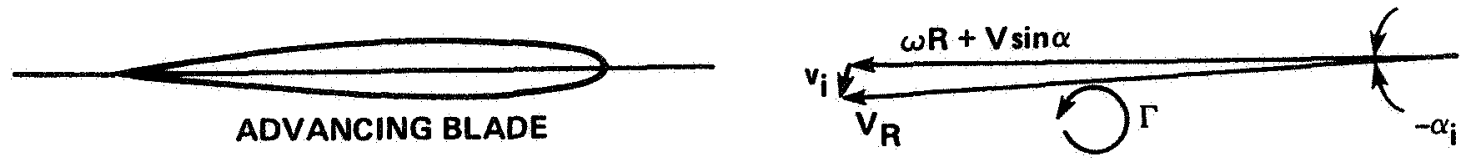

Figure 8.- Blade-vortex interaction on an advancing side.

A conceptual view of the angle-of-attack time-history as the vortex passes near the airfoil is shown in figure 9.

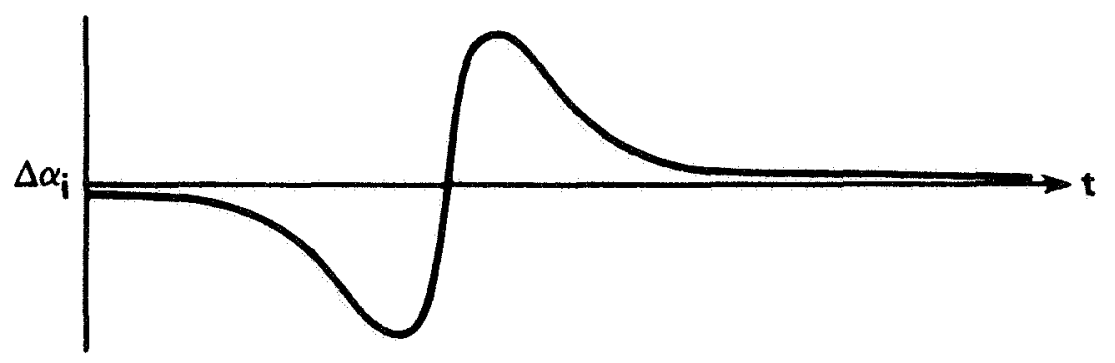

Figure 9.- Angle-of-attack time-history for an advancing blade.

For incompressible flow, this will result in a net lift versus time on the rotor, which is sketched in figure 10 .

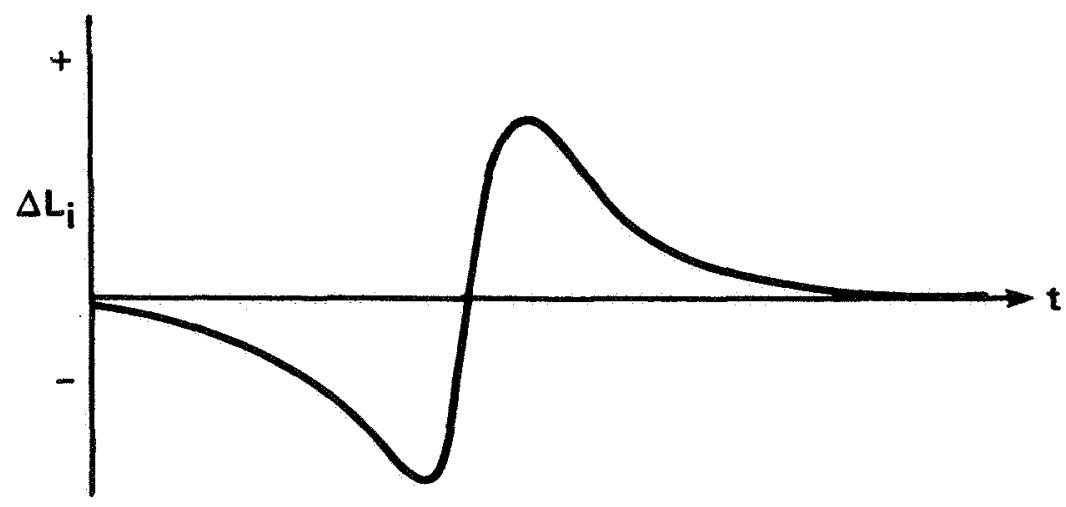

Figure 10.- Lift history of an advancing b1ade. 
In these simple arguments, the entire blade is assumed to feel the presence of the changing angle of attack. The resulting time-varying force field is impulsive in nature. The radiated noise is given by

$$
P^{\prime}(\vec{x}, t)=-\frac{\partial}{\partial x_{i}} \iint\left[\frac{P_{i j} n_{j}}{r\left|1-M_{r}\right|}\right]_{\tau} d S(\vec{n})
$$

Treating the entire blade as a single radiating body (an acoustically compact body) and considering radiation to the far-field, this expression can be rewritten as

$$
P^{\prime}(\vec{x}, t) \approx \frac{\partial}{\partial t} \frac{1}{a_{0}}\left[\frac{\Delta L_{i} \cos \theta_{i}}{r\left|1-M_{r}\right|}\right]
$$

where $\theta_{i}$ is the angle between the surface normal in the direction of the force on the fluid and a line from the point of the applied force to the observer.

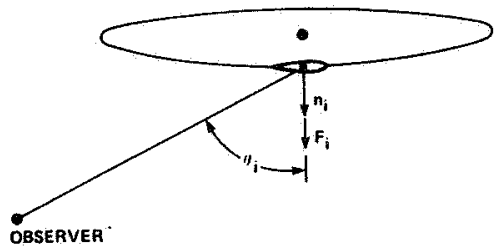

This expression governs the shape of the BVI noise. Similar to thickness noise, the "Doppler amplification" alters the magnitude of the radiation force field, but not the basic character. Thus, the shape of the radiated acoustic pressure becomes that shown in figure 11 .

The net effect of blade-vortex interaction disturbances on the advancing side of the rotor disk is acoustic radiation in the form of a sequence of predominantly positive spikes similar to the above. These near discontinuities are of varying strengths and occur between $\psi=0^{\circ}$ and $\psi=90^{\circ}$. For the observer in the far-field, these positive pressure impulses arrive before the large negative thickness-noise pulse. Notice that the acoustic radiation decreases as the observer approaches the plane of the rotor disk (i.e., $\cos \theta_{i} \rightarrow 0$ ). 


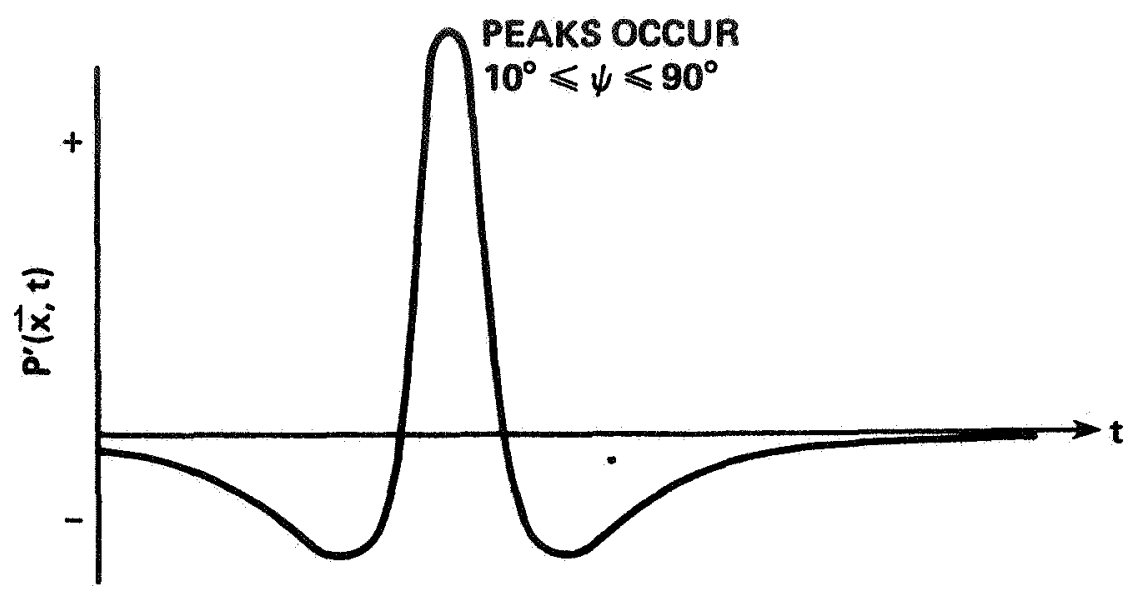

Figure 11.- Acoustic pressure signature of blade-vortex interaction.

Now, consider the BVI on the retreating side of the rotor disk (Nos. 5, 6, 7). Again, using our simplified two-dimensional qualitative model, we have the following geometry:
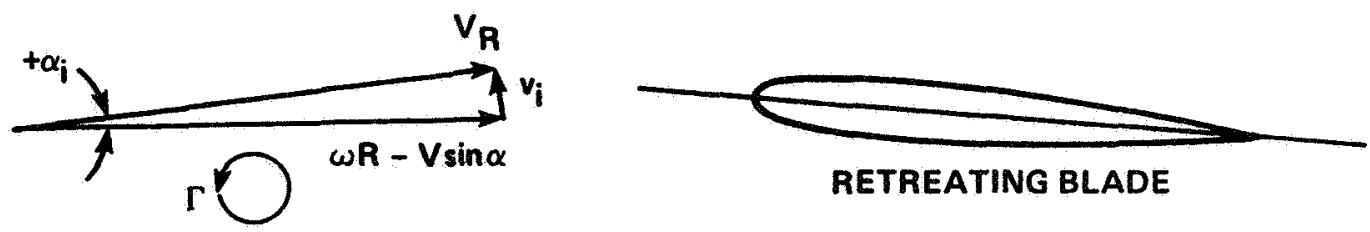

RETREATING BLADE

For retreating BVI, the sign of the approaching vortex is opposite to BVI on the advancing side. Therefore, by similar arguments the net effect is a predominantly negative radiated acoustic signature for each BVI on the retreating side, as shown in figure 12. A11 but the No. 5 BVI impulses occur later in time than the thickness-noise pulse. These two pieces of information help isolate the general origins of the radiated noise. Obviously, these simple qualitative arguments do not tell us many of the more interesting details. However, these arguments do help us interpret measured impulsive-noise acoustic data. (Similar arguments can be found in ref. 10.) 

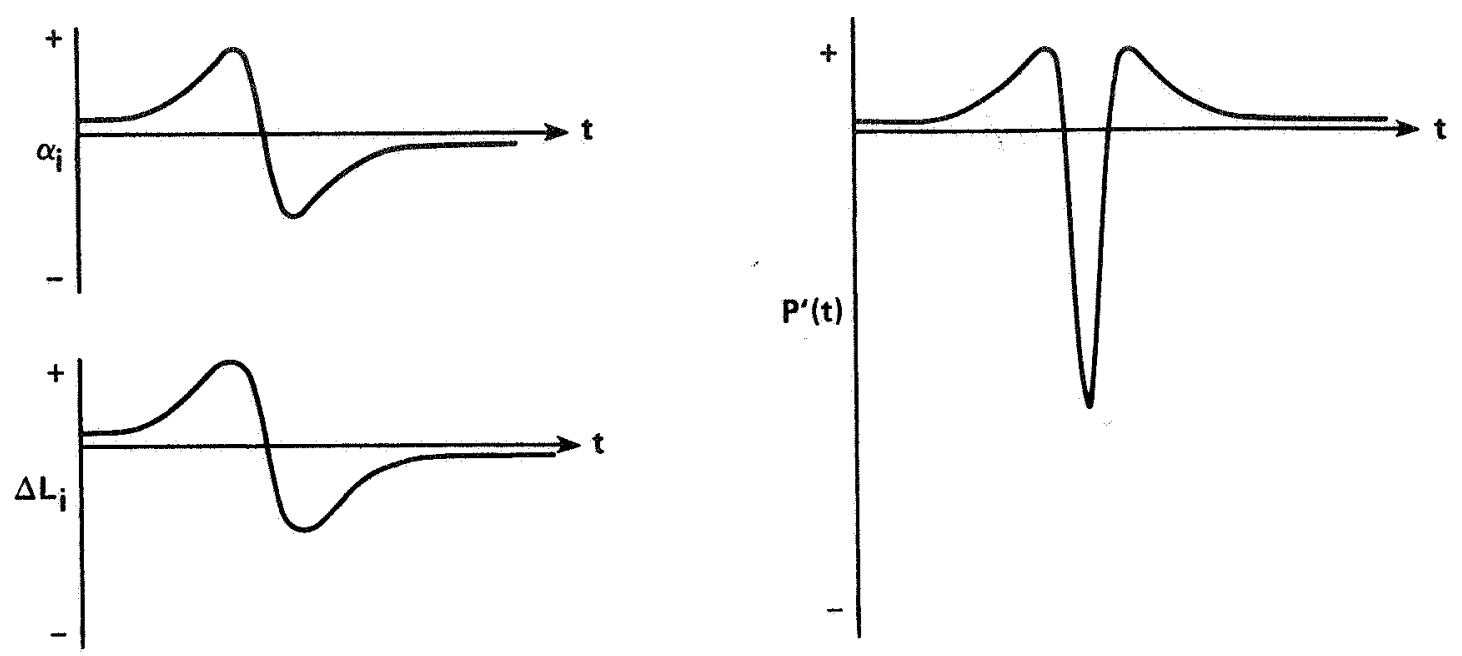

Figure 12.- Angle of attack, lift, and acoustic pressure time history of a retreating blade.

MEASURED IMPULSIVE NOISE

Over the past 10 years, a novel in-flight method of gathering helicopter impulsive-noise data was developed by the Aeromechanics Laboratory (U.S. Army Research and Technology Laboratories, AVRADCOM). In the early stages of the work, an OV-1C Mohawk aircraft was instrumented with a microphone on the top of its vertical tail and flown in formation with the subject helicopter, as shown in figure 13. Because the Mohawk was fairly quiet and the impulsive signals quite intense, good-quality acoustic data were acquired. (Additional details can be found in ref. 11.) The major advantages of gathering data in this manner are (1) no ground reflections; (2) long and steady data samples; and (3) helicopter flight conditions and directivity profiles are easily explored.

The helicopter flight conditions which were investigated for the UH-1H helicopter are shown in figure 14. High-speed impulsive noise was measured in high-speed forward flight, and BVI impulsive noise was measured in moderate forward-speed but descending flight. Also illustrated in this figure are contours of BVI noise as heard in the helicopter cabin. In early experiments, it was thought that cabin noise was a good indicator of radiated impulsive noise. This new in-flight technique showed that cabin measurements were necessary but not sufficient conditions for noise radiation. There is radiated noise in other directions that is not heard in the cabin. Therefore, if a pilot were to fly so as to minimize the cabin impulsive noise, he might still be radiating BVI noise to ground observers. 


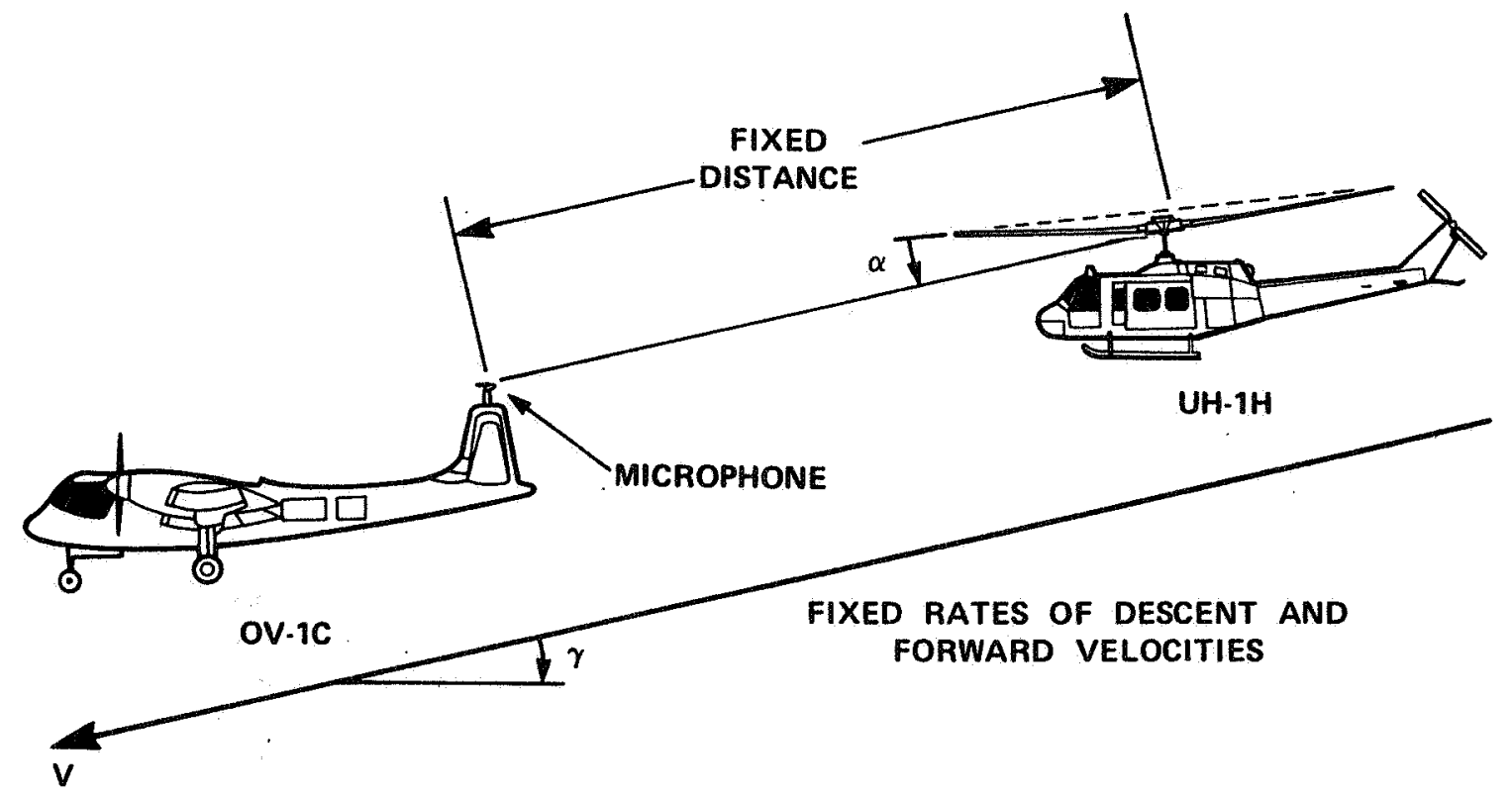

Figure 13.- In-f1ight technique. 


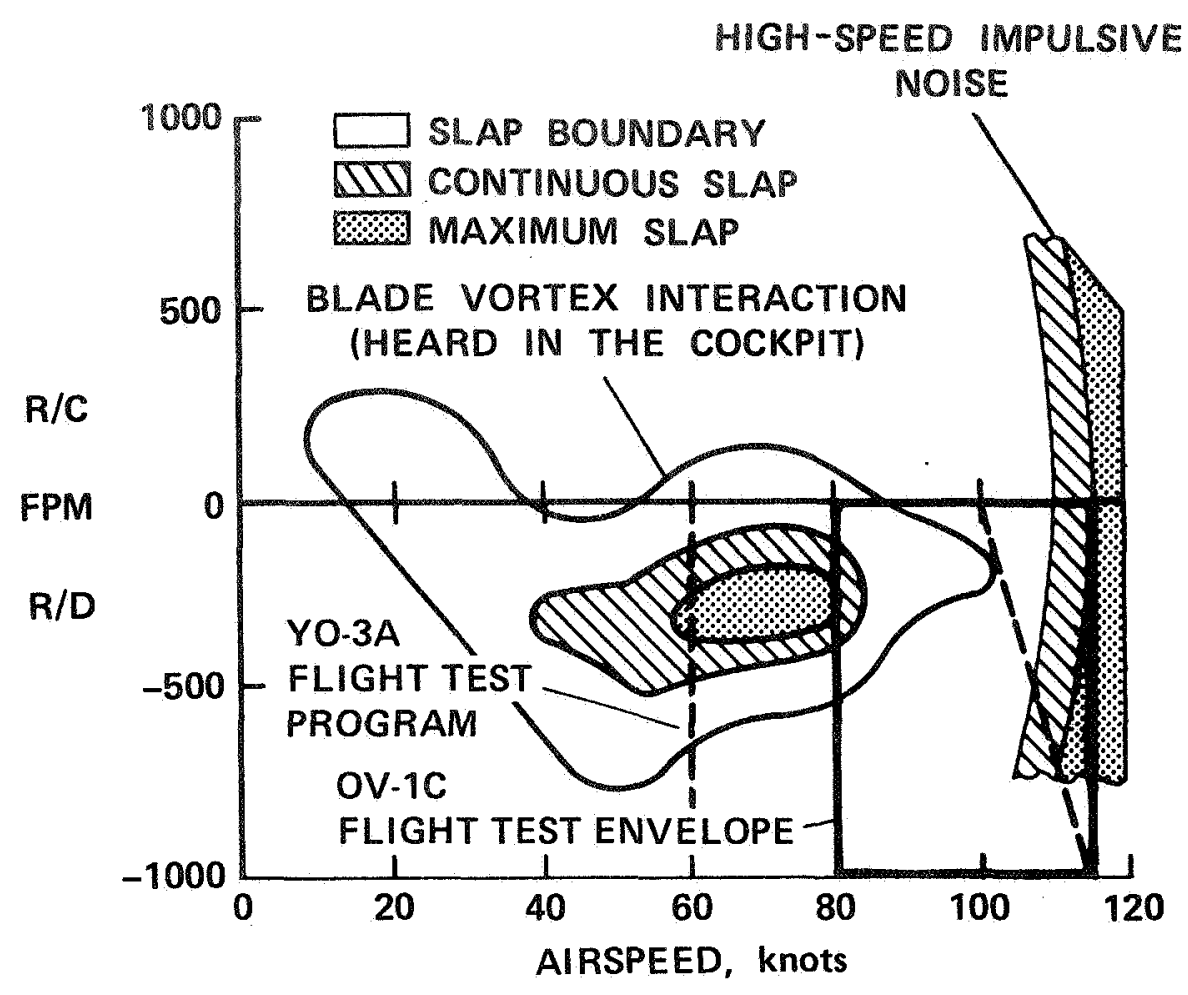

Figure 14.- Impulsive-noise boundaries for UH-1 series helicopters.

It was generally observed from the measured data that the far-field acoustic waveform radiated by each blade was multipulse in nature. As many as three distinct pressure disturbances could be repetitively identified in the acoustic waveform. For identification of this waveform structure and familiarity with data presented in the following sections, an idealized composite drawing of the acoustic waveform showing this multipulse character is presented in figure 15. In this figure, peak pressure amplitude of the acoustic signature is illustrated versus onehalf revolution (one-blade passage) in time, with time increasing from left to right. The peak pressure amplitude scale used here is an absoIute scale measured in dynes per. square centimeter. On this scale, a sinusoida1-shaped waveform with a peak pressure amplitude of 512 dynes $/ \mathrm{cm}^{2}$ would exhibit a root mean square (rms) sound pressure level of $124 \mathrm{~dB}$ (ref. 0.0002 dyne $/ \mathrm{cm}^{2}$ rms).

The composite waveform model illustrates three predominant pressure characteristics observed in the data. They are shown in the same relative sequence and approximate pulse width that were characteristic of the measured data. Typically, the sequence began with one or two successive increases in positive pressure of "triangular" pulse shape (No. 1 in fig. 15). These positive pressure peaks were followed by a large neartriangular negative pressure pulse. At high advance ratios, the negative pressure rise (No. 2) increased in amplitude slightly slower than its subsequent rapid decrease (No. 3), and the waveform is represented more by a saw-tooth or half-triangular pulse. Finally, when it was observed 


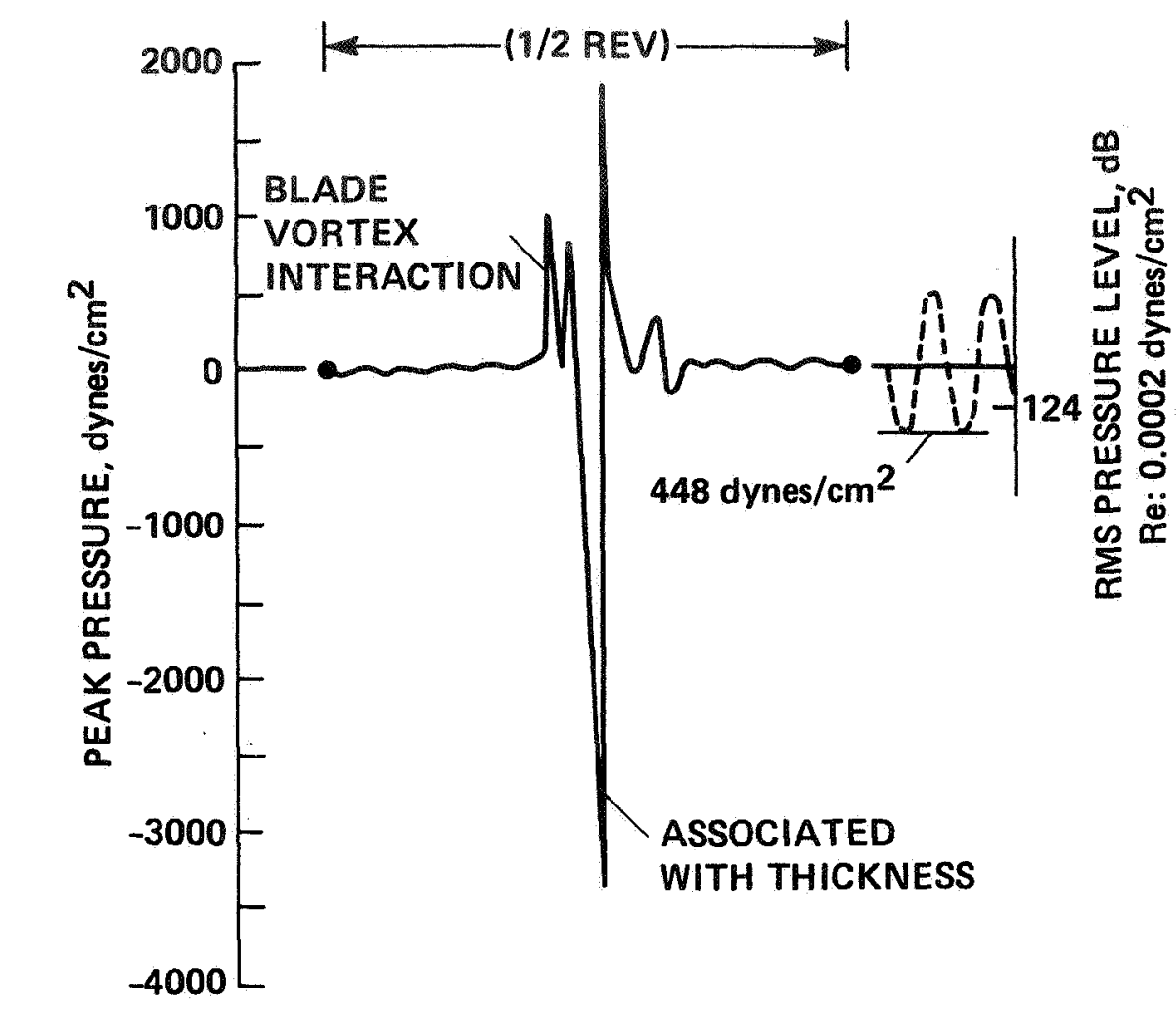

Figure 15.- Composite illustration showing dominant UH-1H acoustic waveform features.

to occur, an extremely narrow positive-pressure spike followed immediately after or as a result of the extremely rapid increase in pressure.

Using the qualitative arguments presented at the beginning of this chapter, it is possible to trace the origins of the radiated noise. Referring to the composite illustration, the negative pulse (No.2, fig. 15) is associated with high-speed thickness effects. As we have shown, it occurs in source coordinates at about $\psi \approx 90^{\circ}$. The initial series of positive pulses (No. 1) is a direct result of blade-tip vortex interaction on the advancing side of the rotor disk. As we have shown, they occur before the thickness-noise impulse, occurring in source coordinates at $\psi \approx 10^{\circ} \rightarrow 80^{\circ}$. Characteristic No. 3 is really just a manifestation of intense thickness noise. It occurs when the thickness noise (and its associated aerodynamics) is so large that local shocks on the blade radiate to the far-field. In this latter case, nonlinear terms need to be added to the simple linear calculations to predict the acoustic far-field.

Figure 16 presents a performance matrix of measured acoustic data at flight conditions between 80 and 115 knots IAS and rates of descent of 0 to $800 \mathrm{ft} / \mathrm{min}$ (see $\mathrm{fig}$. 14). These unaveraged acoustic waveforms, 


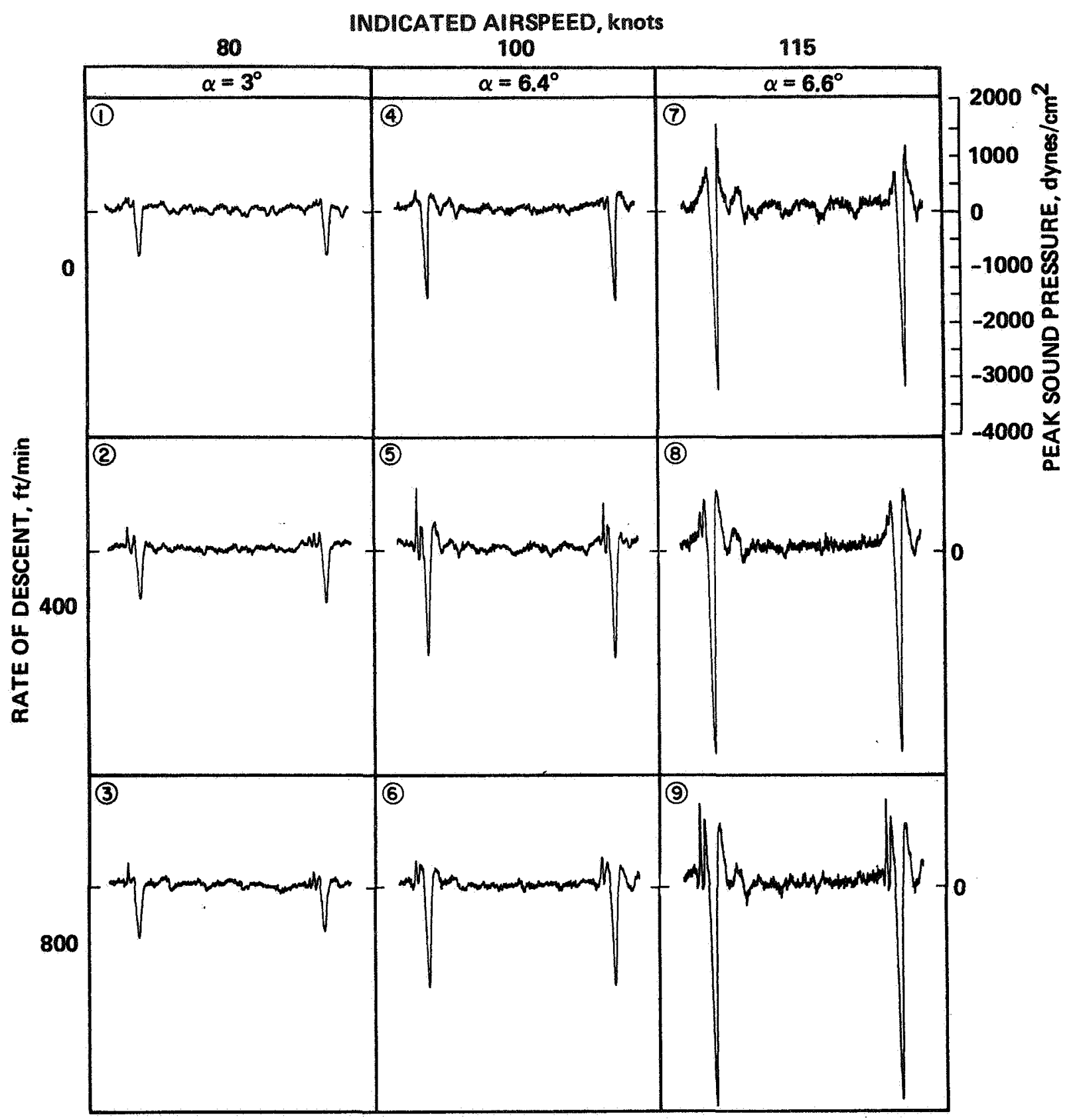

Figure 16.- Unaveraged acoustic signature of UH-1H impulsive noise versus forward airspeed and rate of descent. 
corresponding to two consecutive blade passages, were recorded at a nominal hub-to-microphone separation distance of $95 \mathrm{ft}$ with the microphone positioned directly ahead of the behlciopter $(B=0)$ and nearly within the plane of the rotor tips $(\alpha \approx 0)$. Each of the acoustic timehistories has the same amplitude scale shown, for example, with the upper right waveform in the performance matrix.

To show data trends more clearly, the acoustic waveform for each condition was averaged 128 times, as shown in figure 17 . The large, consistent negative-pressure pulse was used to trigger the averaging process. Since blade-to-blade variability is removed by this technique, only one blade passage is shown. Notice that random background noise has been reduced in level by the averaging process.

Peak amplitude of the large negative-pressure pulse is strongly dependent on forward speed. Although the width of the negative pulse appears to decrease slightly with increasing speed, no consistent trends in amplitude or pulse width could be deduced with changes in descent rate. It is interesting to note that under level-flight conditions at all airspeeds, no impulsive noise was heard in the cabin, indicating that for all flight conditions tested, the pilot was unaware that the helicopter was radiating that part of the impulsive noise waveform associated with the negative pressure peak.

At the high forward speed conditions of 115 knots, the large negativepressure peak, when measured nearly in-plane, was followed by a positivepressure increase which exhibited some variability from blade to blade. This extremely rapid rise in pressure documented here was so intense that it was heard directly in the cockpit of the Mohawk, over and above the aircraft's own internal noise levels. However, no apparent slapping was heard in the cabin of the helicopter at any speed above 100 knots IAS regardless of rate of descent. To the pilot of the helicopter, a moderate increase in vibration level was the only noticeable effect, even though the $\mathrm{UH}-1 \mathrm{H}$ was radiating tremendous amounts of acoustic energy.

Blade slap was heard in the cabin under partial-power descents at forward speeds below 100 knots. Blade slap appeared to be most intense within the helicopter at about 80 knots IAS at a rate of descent of $400 \mathrm{ft} / \mathrm{min}$. The occurrence of this cabin noise correlates with the positive impulse pressures which precede the large negative-pressure pulse on the acoustic waveforms. The occurrence of these positive-pressure pulses is sensitive to rates of descent and resulting rotor-wake geometry, thus confirming that these pulses are a direct result of blade-tip vortex interaction.

Directivity profiles of the UH-1H impulsive noise were measured throughout a sweep of angular microphone positions for two operating conditions: 80 knots IAS, $400-\mathrm{ft} / \mathrm{min}$ rate of descent, and 115 knots IAS, $0-\mathrm{ft} / \mathrm{min}$ rate of descent. The longitudinal and 1ateral angles, $\alpha$ and $\beta$, respectively, were measured from a line in space drawn between the rotor 


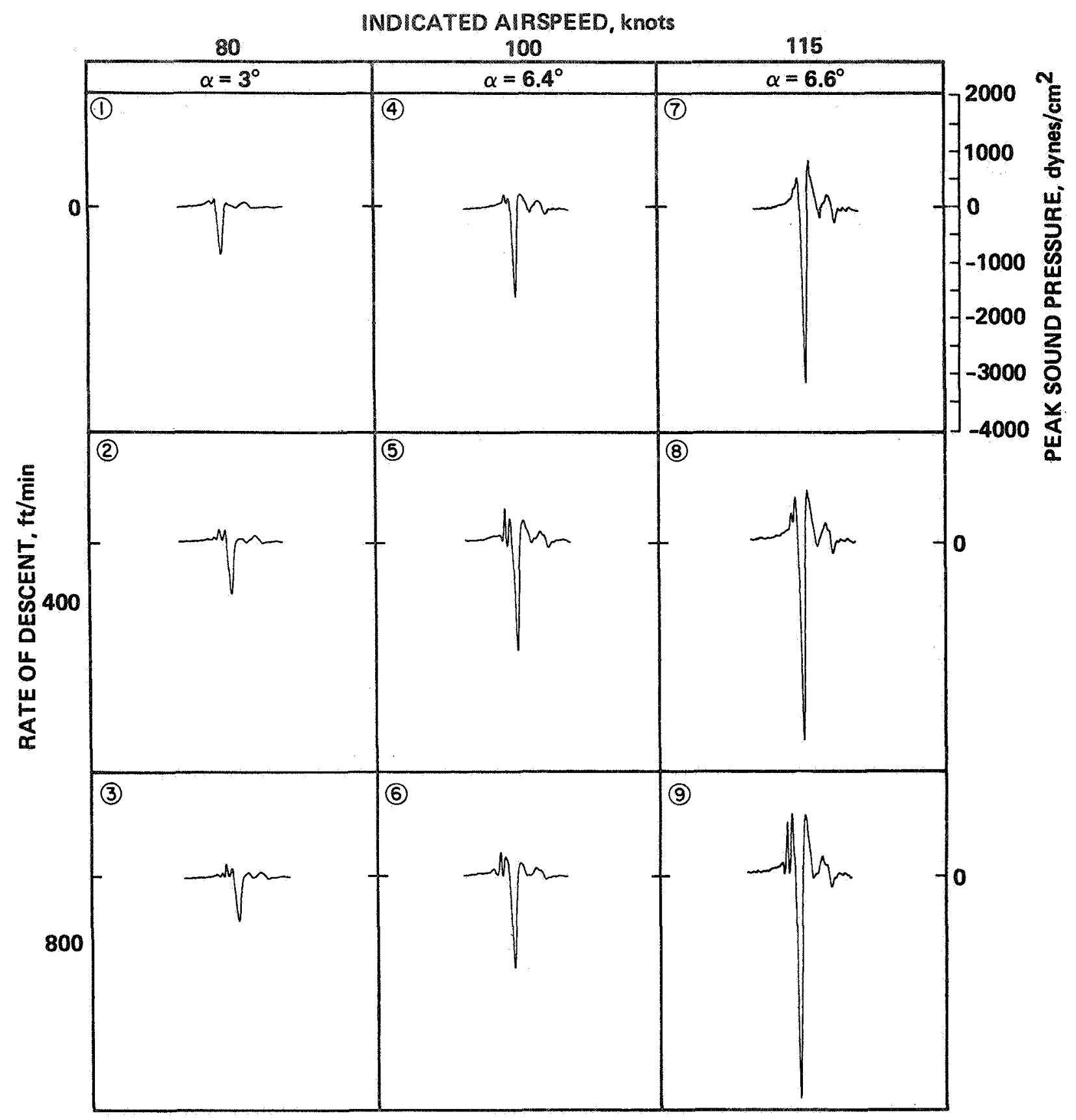

Figure 17.- Averaged acoustic signature of UH-1H impulsive noise versus forward airspeed and rate of descent. 
hub and microphone to the rotor tip-path plane for longitudinal directivity and to the forward velocity vector for lateral directivity.

Figures 18 and 19 present the 1ongitudinal and 1ateral directivity profiles of the $\mathrm{UH}-1 \mathrm{H}$ helicopter in an $80-\mathrm{knot}-\mathrm{IAS}, 400-\mathrm{ft} / \mathrm{min}$ rate of descent. As discussed previously, this operating condition produced blade-slap noise that was audible to the helicopter pilot.

The longitudinal-directivity signatures contain both positive- and negative-pressure pulses, the former exhibiting considerable variability from blade to blade. These positive-pressure pulses, which result because of blade-tip/vortex interaction, become large for longitudinal angles $(\alpha)$ between $10^{\circ}$ and $52^{\circ}$. Even at the nearly in-plane or overhead position, some blade-tip vortex interaction radiation is evident, indicating that a wide angular distribution of acoustic energy is radiated to the far-field in the longitudinal plane. However, the negative pressure peak exhibits quite difrerent radiation characteristics. It reaches its maximum level near the in-plane positions of the rotor disk but decreases rapidly to half amplitude by the $23^{\circ}$ position and continues to decrease uniformly with increasing angle until it is hardly discernible above background noise levels at the $52^{\circ}$ angular position. These experimental findings. are in agreement with the qualitative arguments of the previous section. Thickness noise is a maximum in the plane of the rotor and BVI noise is small at in-plane positions and increases under the rotor.

Lateral-directivity signatures, shown in figure 19, for the same operating conditions reflect a rapid decrease in the impulsive nature of the positive-pressure pulse for measurements to the advancing-blade of the rotor and a gradual disappearance into background noise by the $54^{\circ}$ point. Again, variability between blade signatures is present. The negative-pressure pulse is shown to decay less rapidly in-plane than out-of-plane as the directivity angle is increased. It is approximately half amplitude at $73^{\circ}$ and still discernible to the side of the helicopter $\left(\beta=94^{\circ}\right)$. Although the helicopter pilot cannot hear any slapping noise associated with the negative-pressure pulse, it is clear that near the tip-path plane of the helicopter, large angular distributions of acoustic energy are being radiated.

The longitudinal- and lateral-directivity profiles for the highspeed level flight are shown in figures 20 and 21 . Although the positivepressure pulses associated with blade-tip vortex-interaction impulsive noise are noticeably absent for this flight condition, there are rapid increases first in the large-amplitude negative pressure and then in the positive pressure. The negative-pressure peak is predominantly caused by transonic thickness effects and, although much larger in amplitude for this high-speed condition, varies in longitudinal and lateral directivity in much the same way as in the low-speed case. It is at a maximum near the tip-path plane of the rotor and falls off uniformly with increasing lateral directivity angles where, at $\beta=84^{\circ}$, it is barely noticeable. As indicated previously, the pilot cannot identify this large angular distribution of radiated impulsive noise from inside the helicopter. 


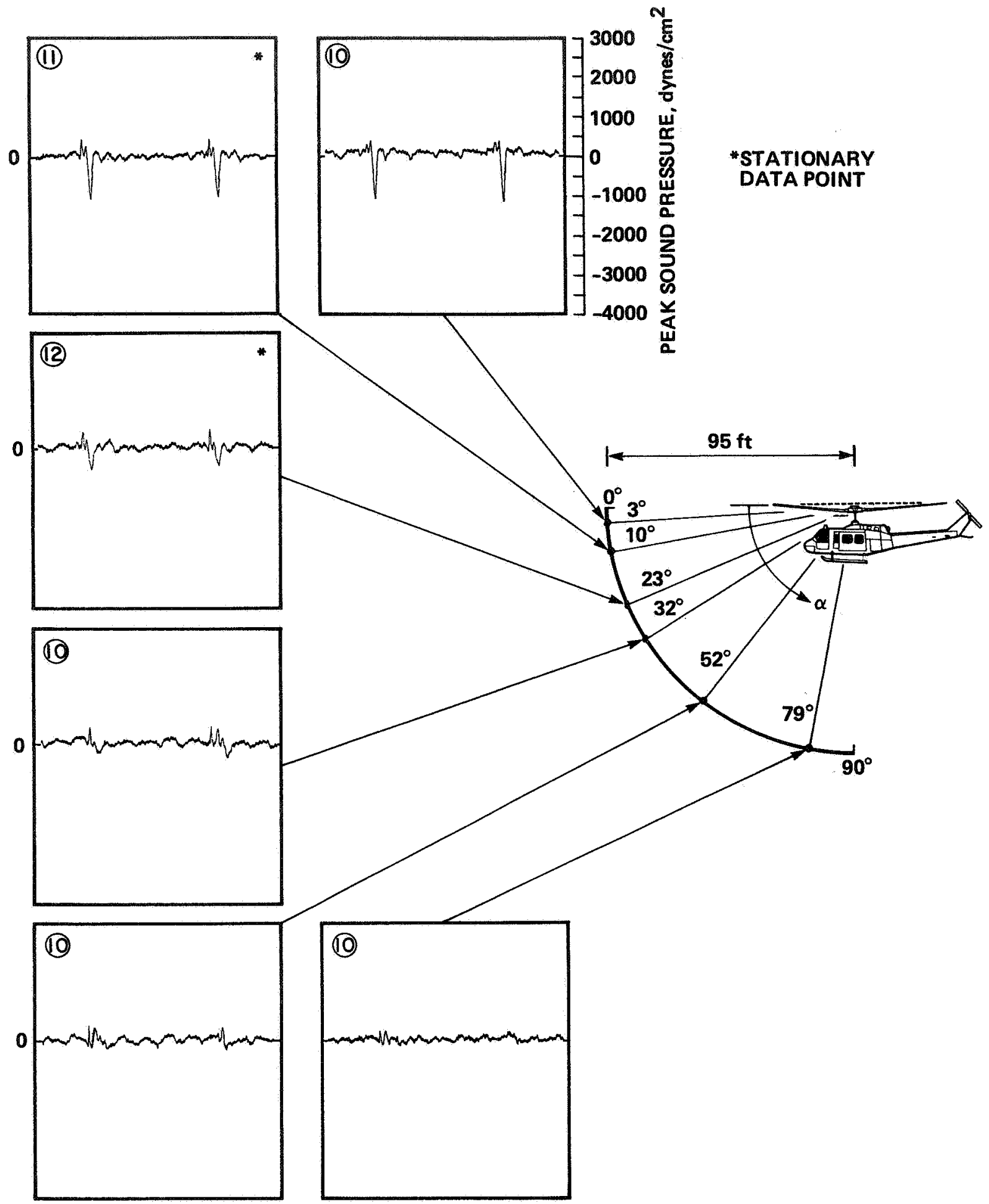

Figure 18.- UH-1H longitudinal acoustic directivity at 80 knots IAS and $400 \mathrm{ft} / \mathrm{min} \mathrm{R} / \mathrm{D}\left(\beta=0^{\circ}\right)$. 


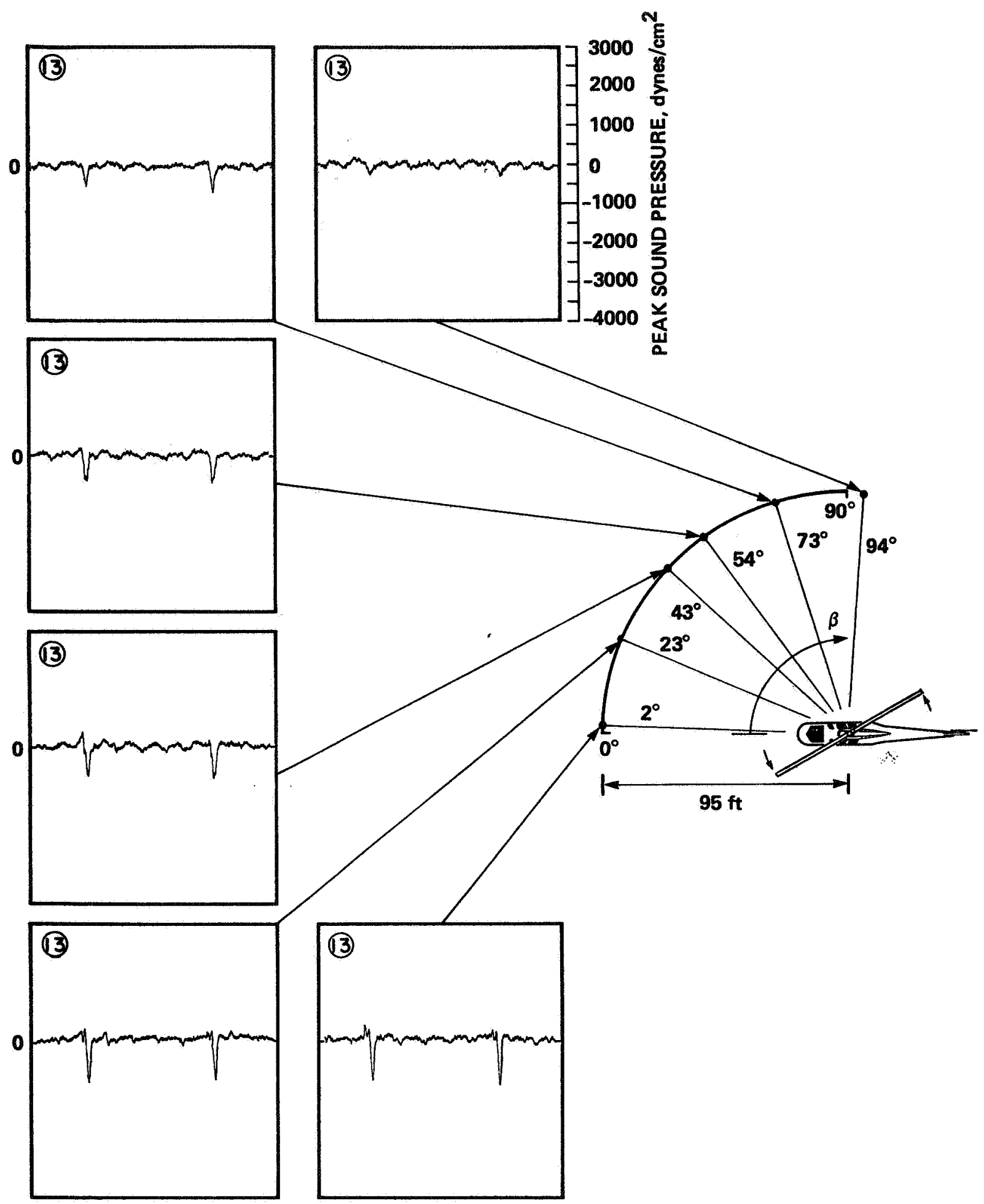

Figure 19.- UH-1H lateral acoustic directivity at 80 knots IAS and $400 \mathrm{ft} / \mathrm{min}$ rate of descent $\left(\alpha=3^{\circ}\right)$. 


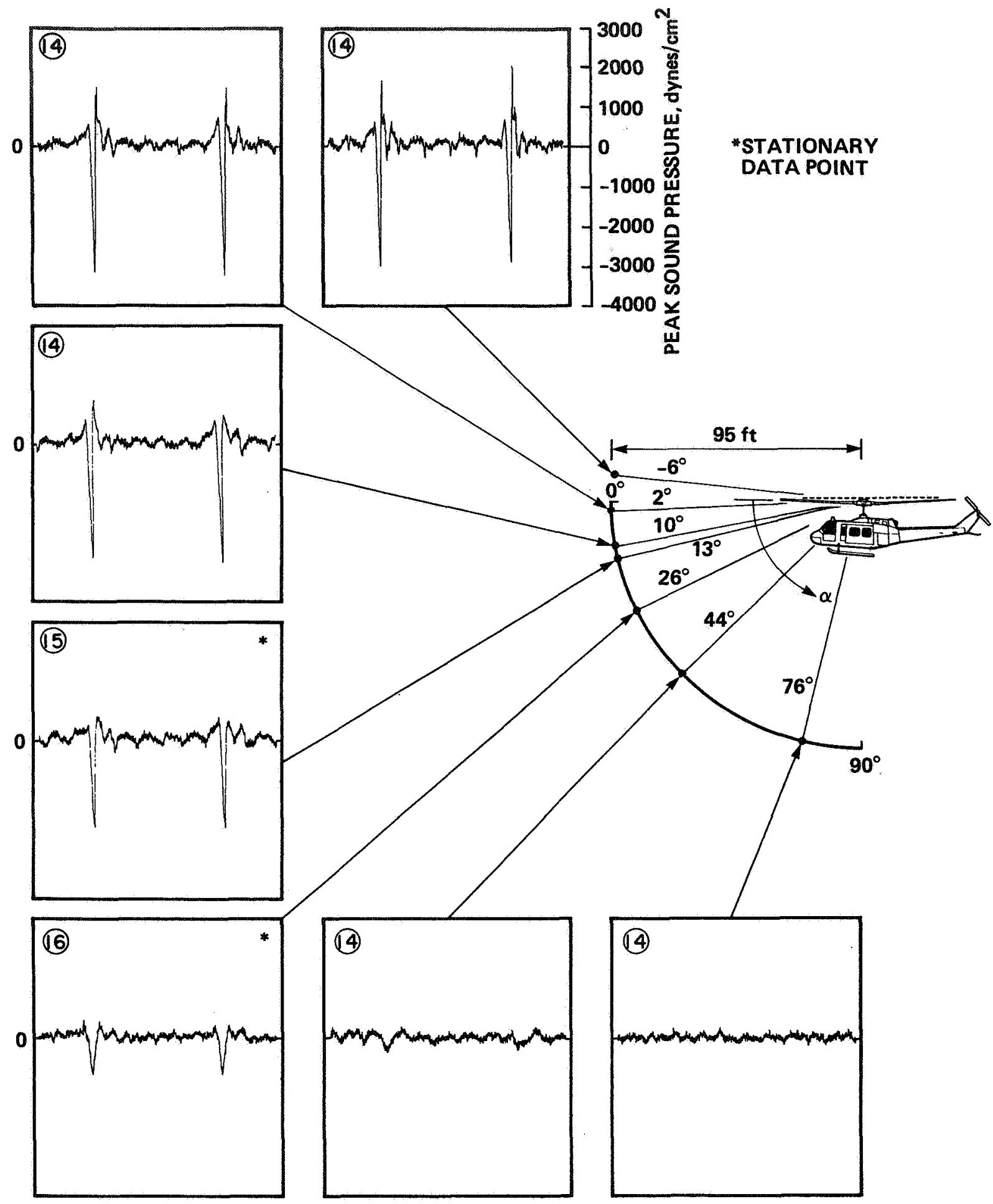

Figure 20.- UH-1H longitudinal acoustic directivity, level f1ight, 115 knots $\operatorname{IAS}\left(\beta=0^{\circ}\right)$. 


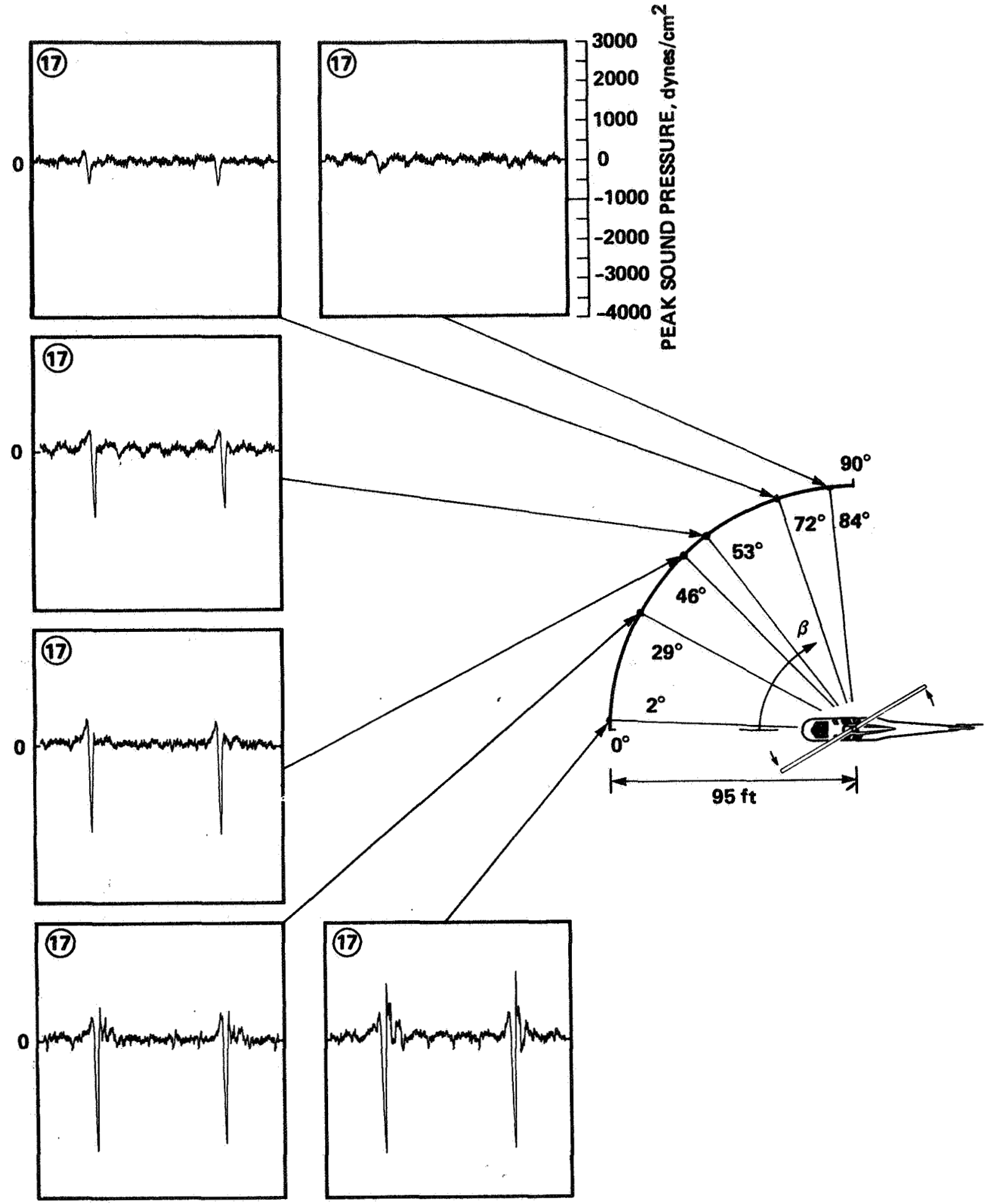

Figure 21.- UH-1H 1ateral acoustic directivity, level flight, 115 knots IAS $\left(\alpha=7^{\circ}\right)$. 
The extremely sharp positive-pressure rise shown to exist in a narrow angular region near and above the rotor's tip-path plane and in the direction of forward flight resulted in very intense radiated noise levels. However, similar to the negative-pressure pulse, no impulsivenoise radiation could be detected by the helicopter pilot. We shall show later that this rapid increase in pressure is associated with a radiating shock wave.

The overwhelming success of the in-flight measurement technique encouraged its further refinement to more quantitatively examine bladevortex interaction (BVI) noise. A specially built "quiet airplane" developed by the Army for Vietnam, called the YO-3A, was employed to gather additional acoustic data on a whole range of helicopters. of course, one of the first tasks was an expansion of the original UH-1H matrix of flight conditions (ref. 12). The Yo-3A could fly more slowly and thus make it possible to investigate fully the conditions of BVI during landing approaches. To emphasize further the signal-to-noise ratios during BVI, the microphone was positioned $30^{\circ}$ below the rotor tip-path-plane, where BVI noise becomes more intense than high-speed impulsive noise (see fig. 22).

A sketch of typical BVI noise is shown in figure 23 for this second test profile with the microphone located $30^{\circ}$ below the tip-path-plane of the rotor. The wide negative-pressure pulse is indicative of high-speed impulsive noise, and the predominately positive-pressure pulses depict impulsive noise resulting from blade-tip vortex interactions. Tail-rotor compressibility noise is also depicted in the same figure by the higherfrequency, smaller-amplitude negative-going pulses.

A matrix of test conditions for the $30^{\circ}$-down microphone position for the UH-1H is shown in igure 24. It is quite apparent that BVI noise plays a larger role in the noise radiation of the $\mathrm{UH}-1 \mathrm{H}$ at this $30^{\circ}$-down position.

Some Impulsive-Noise Power Spectra

Although not the only - or for that matter the most important method of analyzing impulsive noise sources, a power spectral density representation of the characteristic waveforms does indicate in what band of energy the noise is predominant. For this reason, the energy content in each of the three basic waveforms is presented (ref. 11).

Figure 25 illustrates the sound-pressure level of the acoustic signature measured in the far-field near the tip-path-plane $\left(\alpha=3^{\circ}\right)$ for the 80-knot level-flight condition. Frequencies up to $800 \mathrm{~Hz}$ are shown. At higher frequencies, the $\mathrm{UH}-1 \mathrm{H}$ acoustic signature drops into the background noise levels. For reference, the corresponding pressure time-history is also reproduced in the upper right-hand corner of the figure. The dominant feature of this waveform is the negative-pressure pulse that causes the many harmonics of the blade-passage frequency. Most of the energy in 

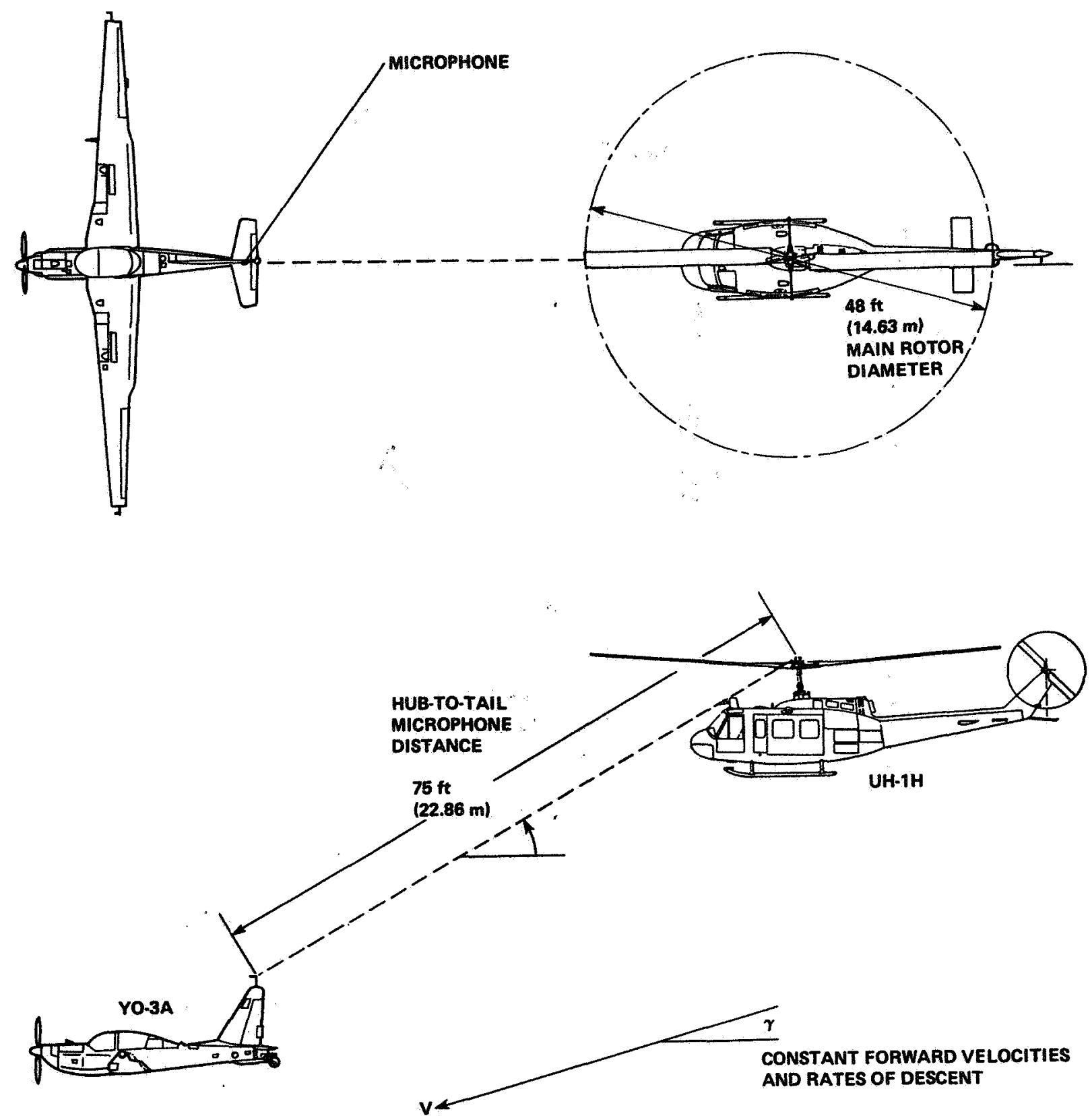

Figure 22.- Relative orientation of aircraft for acoustic measurements of $\mathrm{UH}-1 \mathrm{H}$ blade-vortex interaction noise. 


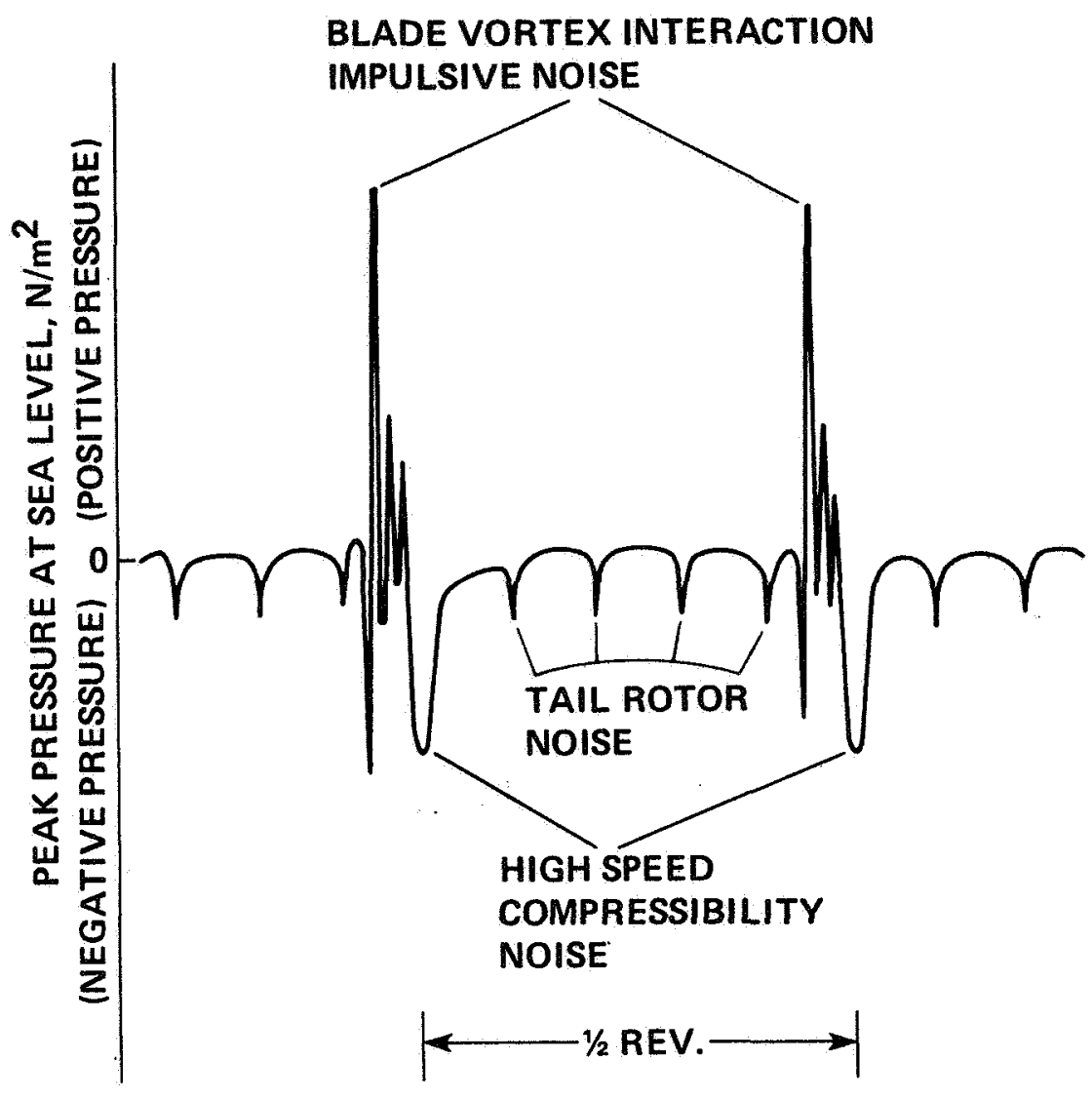

Figure 23.- Composite drawing showing dominant acoustic waveform features. 


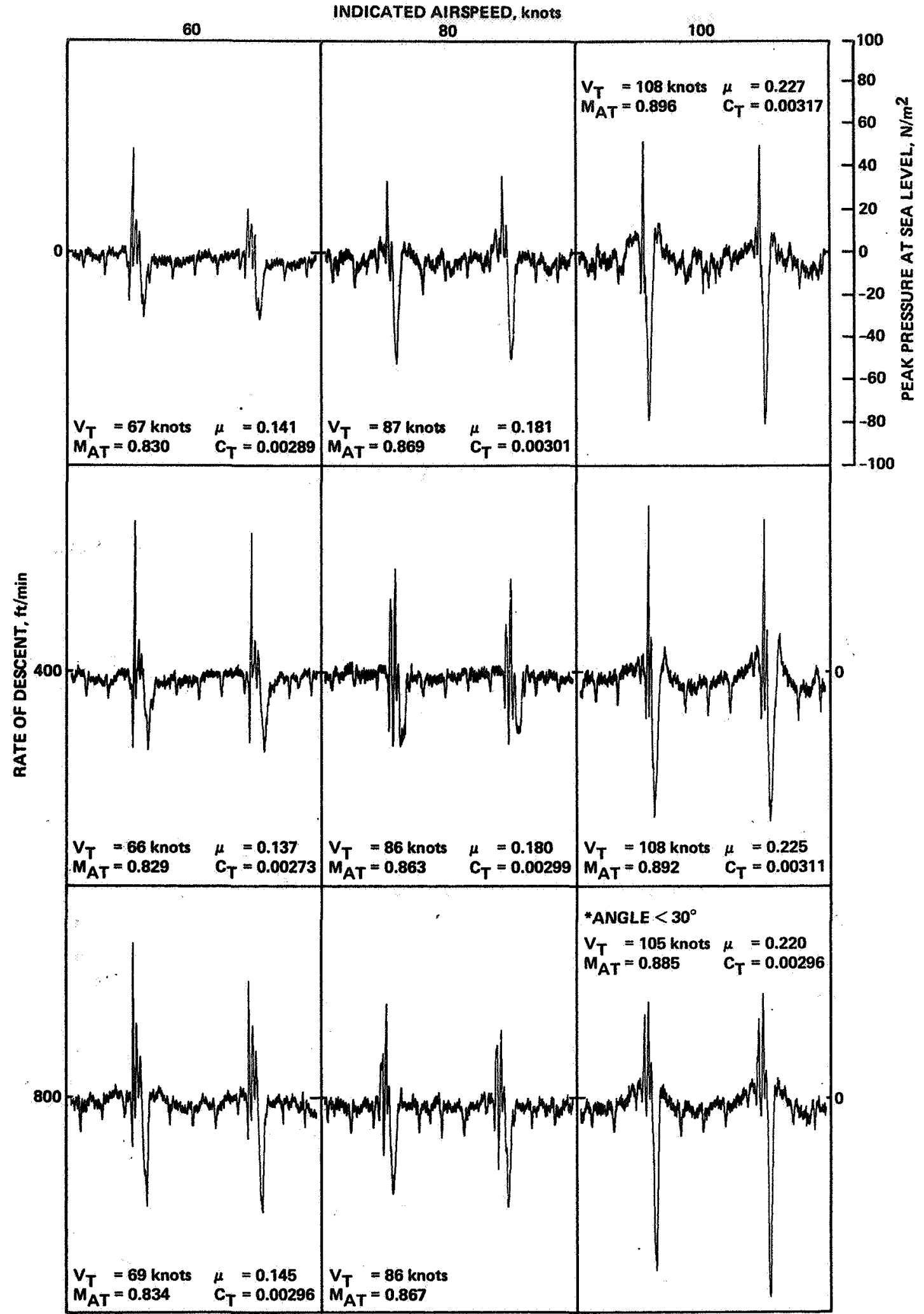

Figure 24.- Unaveraged acoustic signature of UH-1H vs forward airspeed and rate of descent. 


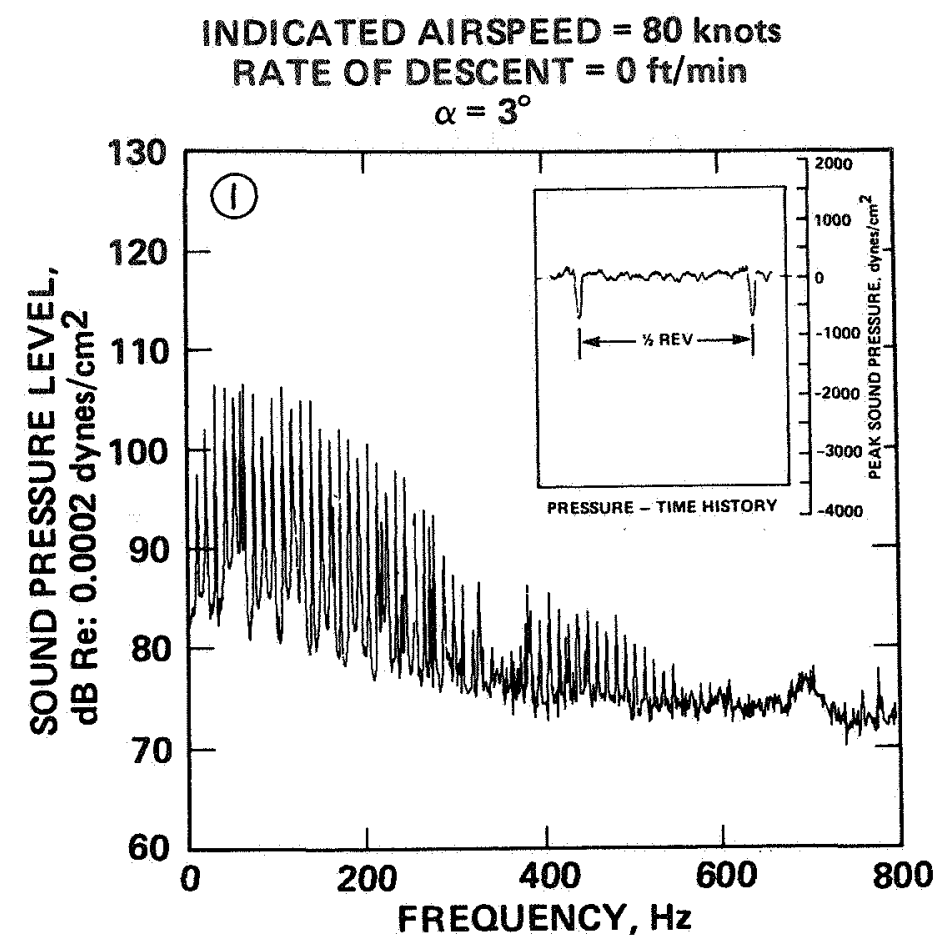

Figure 25.- In-plane UH-1H acoustic power spectrum for level flight, 80 knots IAS: $\alpha=3^{\circ}, \beta=0^{\circ}$.

this pulse is contained in the low-frequency harmonics, up to about $150 \mathrm{~Hz}$. The higher harmonics decrease at a more rapid rate, being $20 \mathrm{~dB}$ down at $300 \mathrm{~Hz}$. Subjectively, this negative-pressure pulse signature can be described as a smooth, loud thumping sound.

The additional harmonics generated as a result of blade-vortex interaction are shown in figure 26. The waveform, which was measured during descending flight $(400 \mathrm{ft} / \mathrm{min})$ at the 80 -knot airspeed, illustrates the occurrence of positive-pressure pulses that precede the larger negativepressure rise. Their major contribution to the power spectrum is in the higher harmonics of blade-passage frequency $(300$ to $600 \mathrm{~Hz})$. Although the energy content of these pulses is considerably less than that of the negative-pressure disturbance, their dominant presence at the higher frequencies is believed to be responsible for the crisp popping or slapping character in the audible acoustic signature.

The last power spectrum, figure 27, illustrates the sound-pressure level of the in-plane far-field acoustic signature for the high-speed, 115-knot level-flight condition together with the measured waveform. A striking increase in acoustic levels at these forward airspeeds is shown out to $1600 \mathrm{~Hz}$. Even though the $\mathrm{UH}-1 \mathrm{H}$ acoustic signature exceeded background noise levels by at least $10 \mathrm{~dB}$ beyond these frequencies, the data began to exceed the dynamic range of the tape-recording equipment and became lost in electronic noise. Although it is difficult to separate rigorously the energy contribution of the large negative-pressure pulse from the following sharp increase in positive pressure, some general 


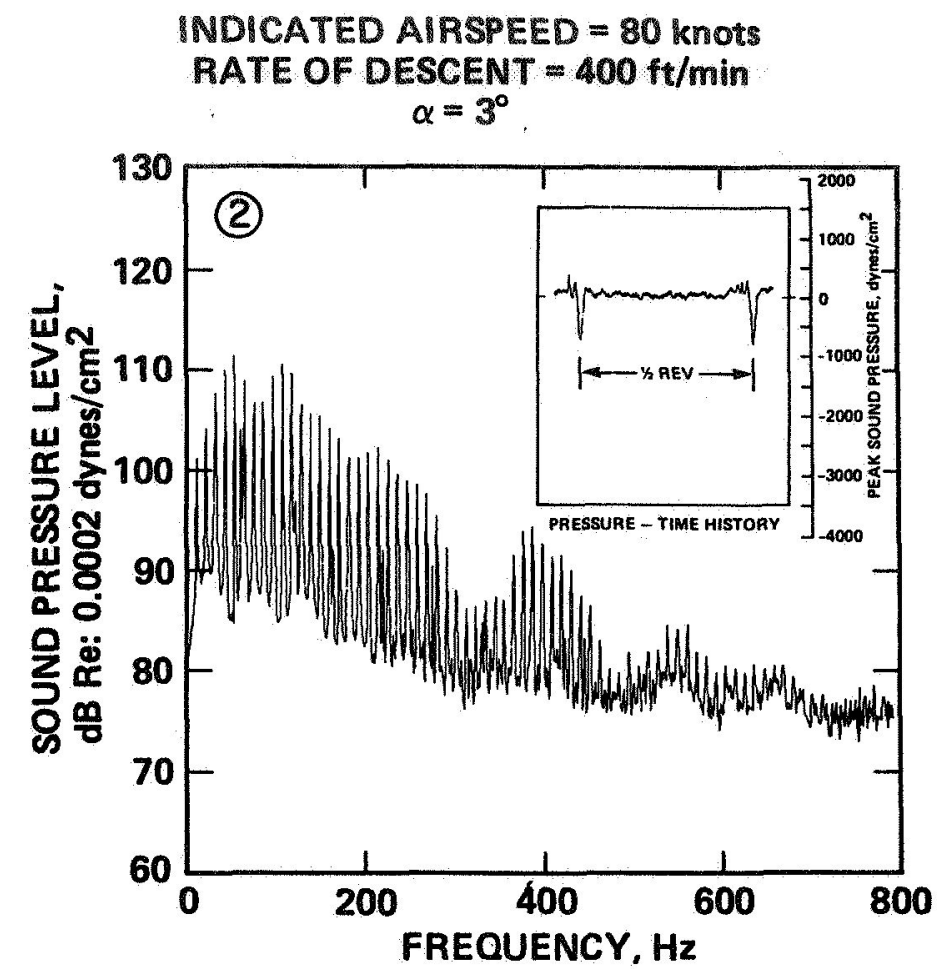

Figure 26.- In-plane UH-1H acoustic power spectrum, descending flight, 80 knots IAS, and $400 \mathrm{ft} / \mathrm{min} \mathrm{R} / \mathrm{D}: \alpha=3^{\circ}, \beta=0^{\circ}$.

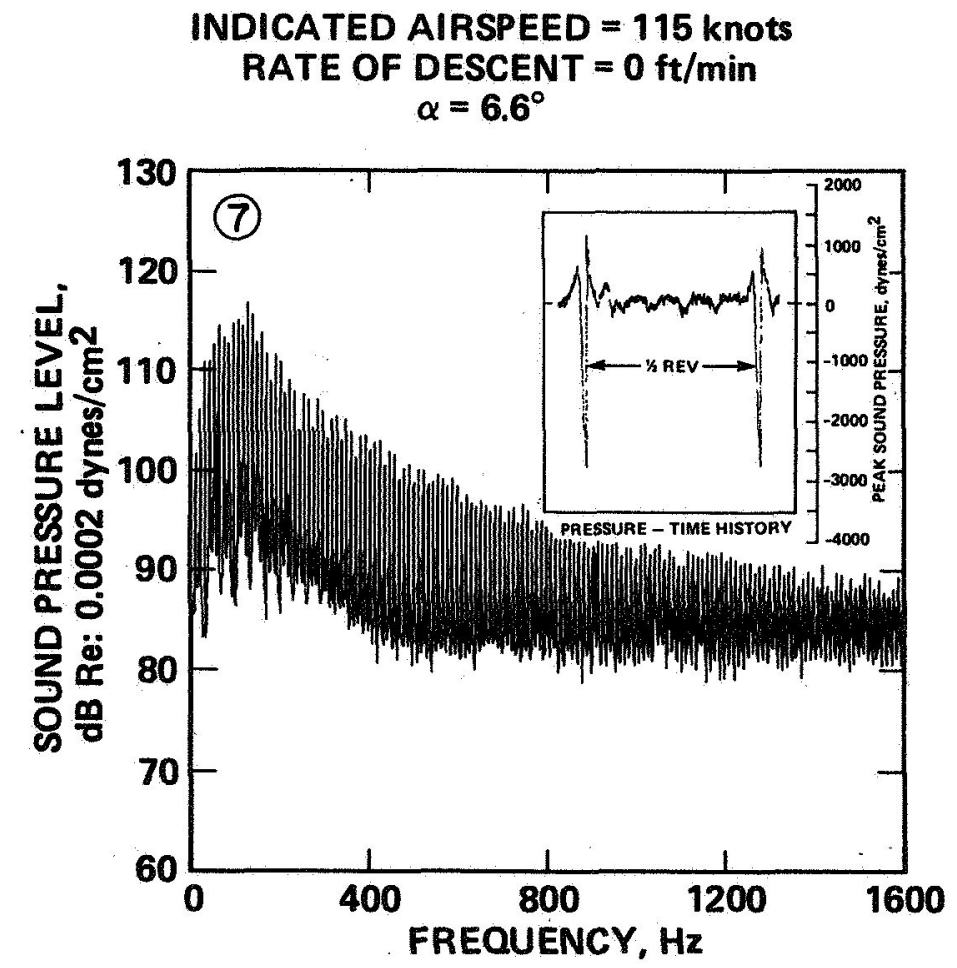

Figure 27.- UH-1H acoustic power spectrum, level flight, 115 knots IAS: $\alpha=6.6^{\circ}, \beta=0^{\circ}$. 
observations can be made. The energy at the low harmonics, as in the low-speed cases, is believed to result primarily from the presence of the large negative-pressure pulse. The sharpness and magnitude of the following increase in pressure causes most of the higher frequency noise shown. As noted previously, the resulting acoustic signal is very intense and definitely dominates all other noises generated by the helicopter.

All of the data shown so far were taken on the UH-1H helicopter. The key operational and design characteristics are shown in table 1. The main point to note is the high main-rotor tip speed. In fact, the high tip speed is responsible for the clean high-level impulsive signals shown. It enabled good signal-to-noise levels to be maintained throughout the test program.

TABLE 1.- UH-1H OPERATIONAL CHARACTERISTICS

\begin{tabular}{lcc}
\hline Rotor system design variables & Main rotor & Tail rotor \\
\hline Number of blades & 2 & 2 \\
Rotor diameter, ft & 48 & 8.5 \\
Rotor solidity & 0.0464 & 0.105 \\
Blade chord, in. & 21 & 8.41 \\
Blade airfoil & NACA 0012 & NACA 0015 \\
Blade twist (root to tip), deg & -10.9 & 0 \\
\hline Aircraft operational 1imits & Maximum & Minimum \\
\hline Main-rotor tip speeds, ft/sec & 813.8 & 740.0 \\
Tail-rotor tip speeds, ft/sec & 736.1 & 669.0 \\
Forward flight airspeeds, knots & 115 & 0 \\
Gross weight, lb & 9500 & 6600 \\
\hline
\end{tabular}

Over the years, the technique of using a "flying microphone" has been used by the Aeromechanics Laboratory to measure the noise of many helicopters. More recently, Ames Research Center acquired a Yo-3A and has dedicated it to the helicopter-noise measuring role. The various acoustic signatures of all kinds of single-rotor helicopters have more similarities than differences. For example, acoustic data from a modern four-bladed helicopter in the in-plane in-trail position is shown in figure 28. The helicopter is in level flight at 130 knots IAS. Notice that the mechanisms are quite similar to those of two-bladed rotors. A sequence of positive BVI impulses precedes the high-speed thickness-noise expansion. However, instead of two characteristic waveforms per rotor revolution, we now see four, corresponding to the fourbladed rotor system.

This particular helicopter has a much lower main-rotor hover tip speed than the $\mathrm{UH}-1 \mathrm{H}(\Omega \mathrm{R} \cong 700 \mathrm{ft} / \mathrm{sec})$. However, as in most modern helicopters, it also cruises at much higher velocities. The net result 


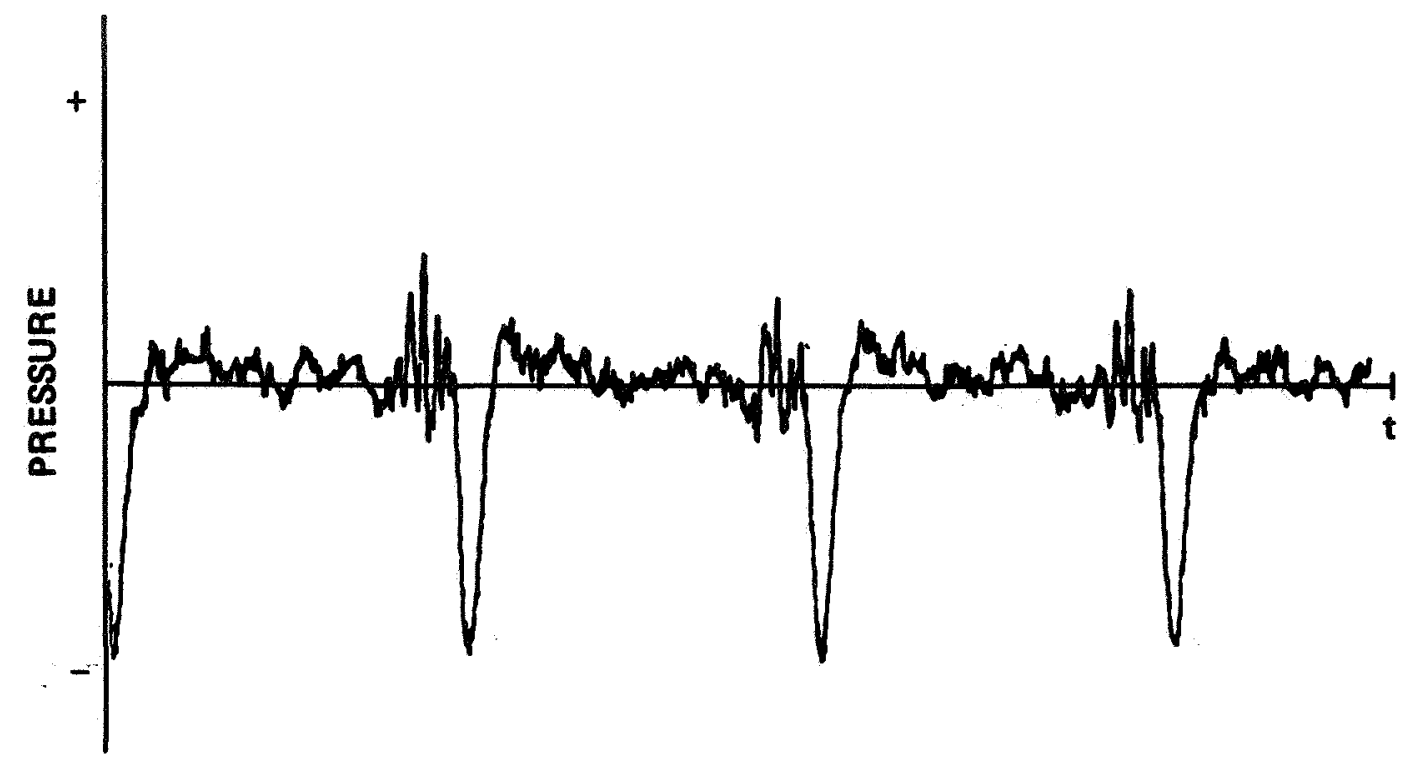

Figure 28.- Acoustic signature of a modern four-bladed helicopter.

is a quite comparable advancing-tip Mach number on both helicopters in cruise. Therefore, it is not too surprising to find that the acoustic radiation is quite similar in character. As we have seen, the advancingtip Mach number plays a key role in all helicopter acoustic problems.

HIGH-SPEED IMPULSIVE NOISE

Now that we have reviewed the necessary mechanisms for impulsive noise on two- and four-bladed helicopters, let us look more closely at the detailed aerodynamic origins of high-speed impulsive nolse. In particular, we pose four fundamental questions and attempt to answer them in the following sections:

1. Can high-speed impulsive noise be scaled, and, if so, what are the scaling parameters?

2. Is the rapid high-speed impulsive noise compression which follows the expansion region at high advancing-tip Mach numbers a radiating (delocalized) shock wave, and can it be measured?

3. Can high-speed impulsive noise be duplicated in a steady environment (i.e., how important are the unsteady effects)?

4. How accurately can high-speed impulsive noise be predicted? What degree of modeling is required? 
Our theoretical results so far have shown that a very few key nondimensional variables control much of the acoustic radiation. The fact that high-speed impulsive noise is predominately a noncompact (source and sinks do not completely cancel for an in-plane observer) high-Machnumber (compressible) event would suggest that small models could be made to duplicate the full-scale acoustic phenomena. This fact was demonstrated in two separate wind-tunnel experiments (refs. 13 and 14). In the first, a $1 / 7$ scaled $\mathrm{UH}-1 \mathrm{H}$ model rotor was fabricated and run in an acoustically treated wind tunnel. The data were compared with full-scale data taken at similar nondimensional conditions. As illustrated in figure 29, modelto-full-scale comparisons are quite straightforward. There are no Doppler corrections and data records up to $1 \mathrm{~min}$ in duration are possible at steady-state flight conditions.

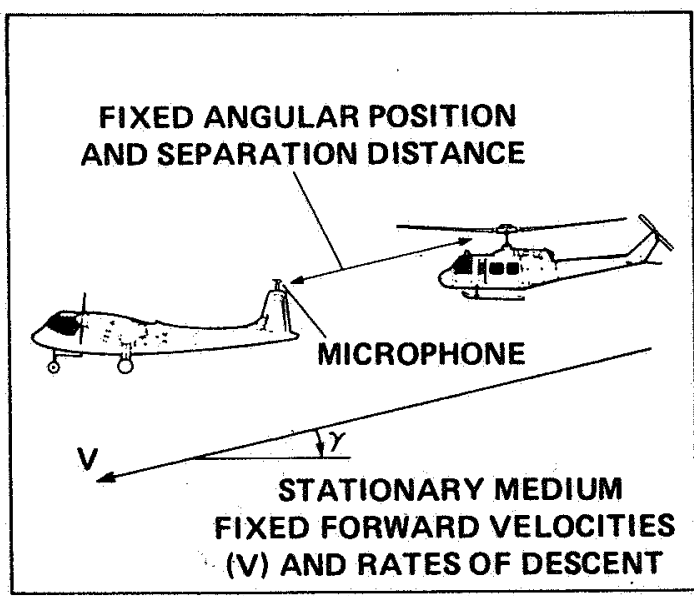

IN-FLIGHT ACOUSTIC TESTING

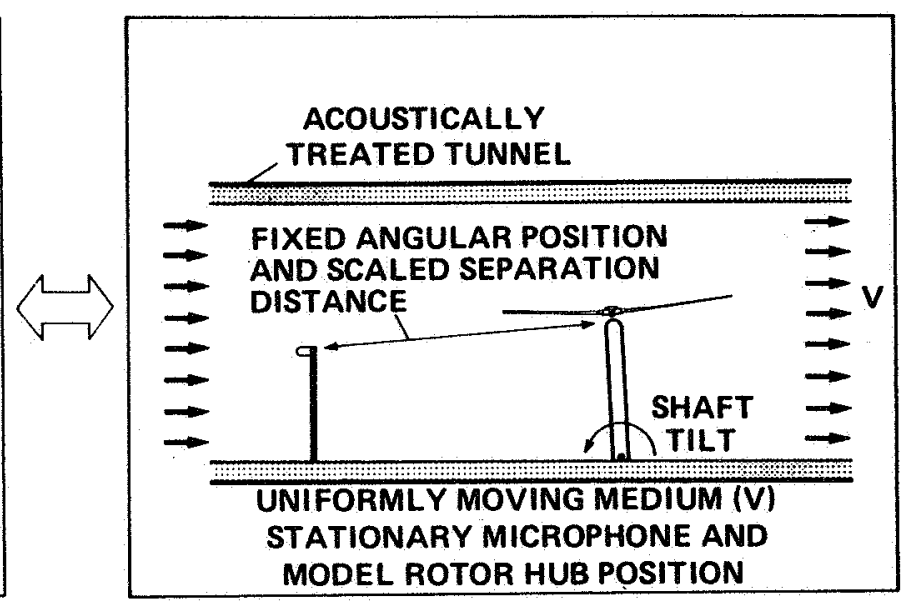

ACOUSTIC TESTING IN A WIND TUNNEL

Figure 29.- Model and full-scale acoustic testing.

From dimensional analysis considerations, we know that there exist nondimensional variables which govern acoustic radiation. If we consider linear thickness noise, ${ }^{1}$ we have (eq. (2))

$$
P^{\prime}(\vec{x}, t)=\frac{1}{4 \pi} \frac{\partial}{\partial t} \iint\left[\frac{\rho_{0} v_{n}}{r\left|1-M_{Y}\right|}\right]_{\tau} d S(\vec{n})
$$

where $t=\tau+r / a_{0}$. But

$$
v_{n}=v \cdot \lambda
$$

\footnotetext{
${ }^{1} \mathrm{~A}$ nondimensionalization of the complete integral equation is given
} in ref. 14 . 
where $\lambda=$ local blade surface slope and $V=$ velocity of the undisturbed flow at each blade station. If we assume geometric scaling and introduce the nondimensional unit of time $\bar{t} \equiv t /(2 \pi / \Omega)$, then

$$
\overline{\mathrm{ds}}=\frac{\mathrm{ds}}{\mathrm{R}^{2}}, \quad \overline{\mathrm{r}}=\frac{\mathrm{r}}{\mathrm{R}}, \quad \overline{\mathrm{t}}=\frac{\mathrm{t}}{2 \pi / \Omega}, \quad \overline{\mathrm{t}}=\bar{\tau}+\frac{\overline{\mathrm{MM}_{t}}}{2 \pi}
$$

when $M_{t}=\Omega R / a_{0}$. Defining $C_{p}(\bar{x}, \bar{t}) \equiv P^{\prime}(x, t) /\left(p_{o} a_{o}^{2}\right)$ (i.e., a nondimensional acoustic pressure), we have in nondimensional notation,

$$
C_{p}^{\prime}(\bar{x}, \bar{t})=\frac{P^{\prime}(\bar{x}, \bar{t})}{\rho_{o} a_{0}^{2}}=\frac{1}{8 \pi^{2}} M_{T} \frac{\partial}{\partial \bar{t}} \iint\left[\frac{M \lambda}{\bar{r}\left|1-M_{r}\right|}\right]_{\bar{r}} d \bar{s}
$$

where $M=v / a_{0}$.

This equation states that if the rotor, microphone geometry, Mach number, and time are scaled, the acoustic-pressure coefficient is uniquely determined. However, most acoustic data are not compared on a nondimensional basis. Instead, all pressures are normally feferred to sea-level standard conditions where comparisons of pressure timehistories are made. For full-scale data taken at altitude, the referred pressure becomes

$$
\begin{aligned}
& \frac{P_{S L}^{\prime}(\bar{x}, t)}{\rho_{O S L} a^{2}}=\frac{P^{\prime}(\bar{x}, \bar{t})}{\rho_{O} a_{O}^{2}} \\
& P_{S L}^{\prime}(\bar{x}, \bar{t})=\frac{\rho_{O S L} a_{O S L}^{2}}{\rho_{0} a_{O}^{2}} P^{\prime}(\bar{x}, \bar{t})=\frac{P_{O S L}}{P_{O}} P^{\prime}(\bar{x}, \bar{t})
\end{aligned}
$$

For model-scale data, the referred pressure becomes

$$
P_{m_{S L}}^{\prime}(\bar{x}, \bar{t})=\frac{P_{o_{m_{S L}}}}{P_{o_{m}}} P_{m}^{\prime}(\bar{x}, \bar{t})
$$

Therefore, by matching the above nondimensional parameters, the referred model-scale acoustic pressures should duplicate the referred full-scale acoustic measurements. This is confirmed in figure 30 for the Uh-1H helicopter in high-speed flight. Notice that the time for one rotor revolution of model-scale data is $\Omega / \Omega_{\mathrm{m}}$ times the time for one revolution of full-scale data. (It should be noted that in this early research, changes in $\rho_{0}$ were accounted for when comparing full-scale pressures to wind-tunnel model acoustic pressures; however, variations in $a_{0}$ were not. It is estimated that corrections for $a_{0}$ could increase the full-scale levels shown by $6 \%$ - thus making the comparison less favorable. However, more recent experimental research with very high quality model and fullscale data shows improved agreement (see ref. 14).) 


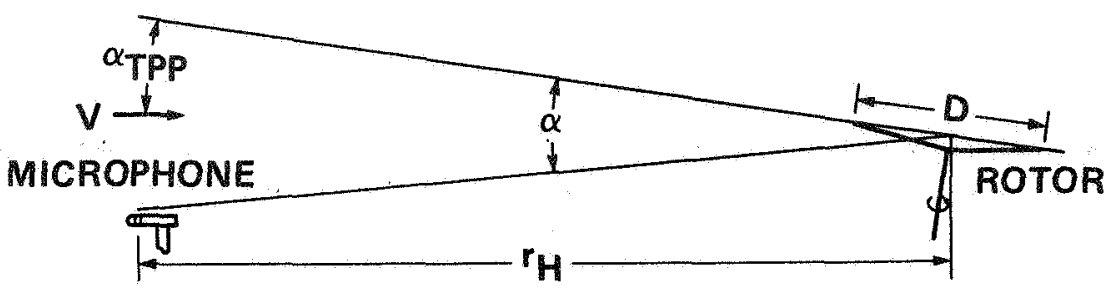

FULL SCALE

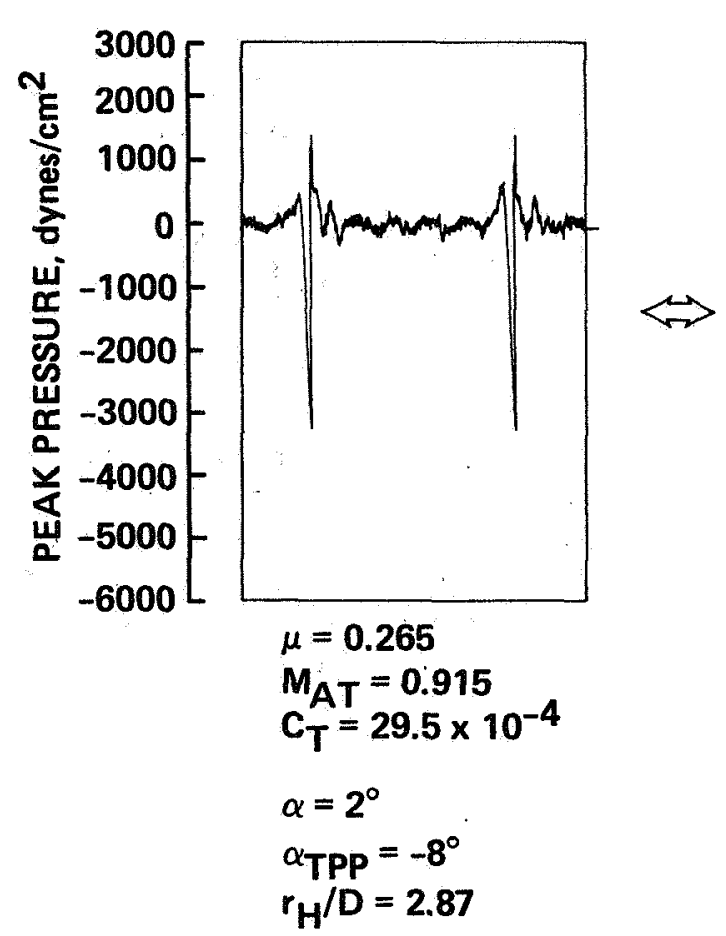

MODEL SCALE

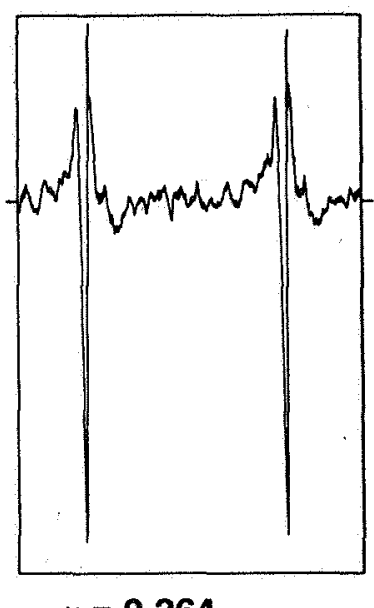

$\mu=0.264$

$M_{A T}=0.924$

$\mathrm{C}_{\mathrm{T}}=29.5 \times 10^{-4}$

$\alpha=2^{\circ}$

$\alpha=2.46^{\circ}$

$r_{H} / D=2.87$

$\alpha_{\text {TPP }}=-2^{\circ}$

$r_{H} / D=1.52$

Figure 30.- Waveform comparison: full-scale and model-scale high-speed data.

It should be noted that the full-scale data were acquired at larger scale nondimensional distances than the model-scale results. If this is accounted for, the peak negative-pressure comparison between model- and full-scale data is quite good, as shown in figure 31. However, the modeldata peak amplitudes are in generally slightly smaller than the full-scale data. The nonanechoic properties of the treated wind tunnel are believed to be partially responsible for this discrepancy.

More recent scaling tests on the AH-1 series helicopter (ref. 14) have resolved most of the mode1-scale/full-scale comparison differences. A nearly perfect match of peak amplitudes and pulse shapes is shown in figure 32. The model-scale acoustic data were taken in the world's largest anechoic wind tunnel (DNW) and are of very high quality. The in-flight acoustic data which are shown were taken with the Yo-3A aircraft. 


$$
r / D=1.52
$$

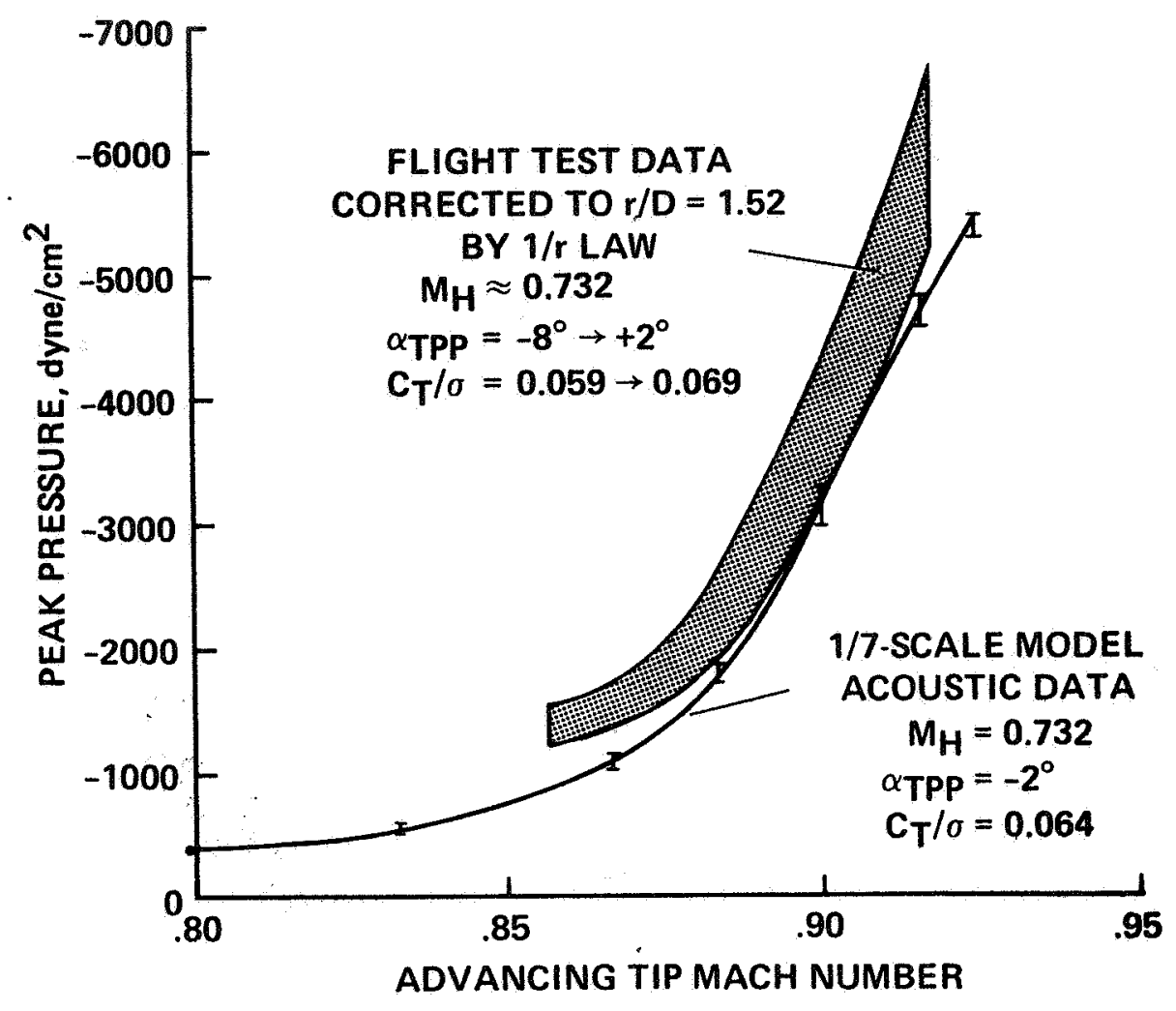

Figure 31.- Peak negative-pressure amplitude comparison: full-scale and model-scale high-speed data.

The pulse shapes of both the model and full scale have been averaged for comparison purposes. (For more details, see ref. 14.)

The model-scale/full-scale comparison is made even more convincing by considering other than the in-plane microphone position. Longitudina1and lateral-directivity comparisons are explored in figure 33 for one advancing-tip Mach number (ref. 14). As shown, agreement is the best at the in-plane microphone positions but is still very good at positions under the horizontal plane of the helicopter. Data from these UH-1 and AH-1 model-scale/full-scale tests demonstrate that carefully designed and nondimensionally tested small-scale models can duplicate the highspeed impulsive noise generated by full-scale rotors.

It is also interesting to note at this juncture that thrust plays only a secondary role in the in-plane high-speed impulsive noise radiation process. Figure 34 presents the variation of peak pressure levels with changes in thrust coefficient for a microphone positioned nearly in-plane with the plane of the rotor for the UH-1 model rotor. The data indicate a remarkable insensitivity to changes in thrust. Notice that the small variations about the mean line of zero slope that do exist are caused by variations in tip Mach number about $\mathrm{M}_{\mathrm{AT}}=0.9$. 
OLS MODEL ROTOR ACOUSTIC SIGNATURES

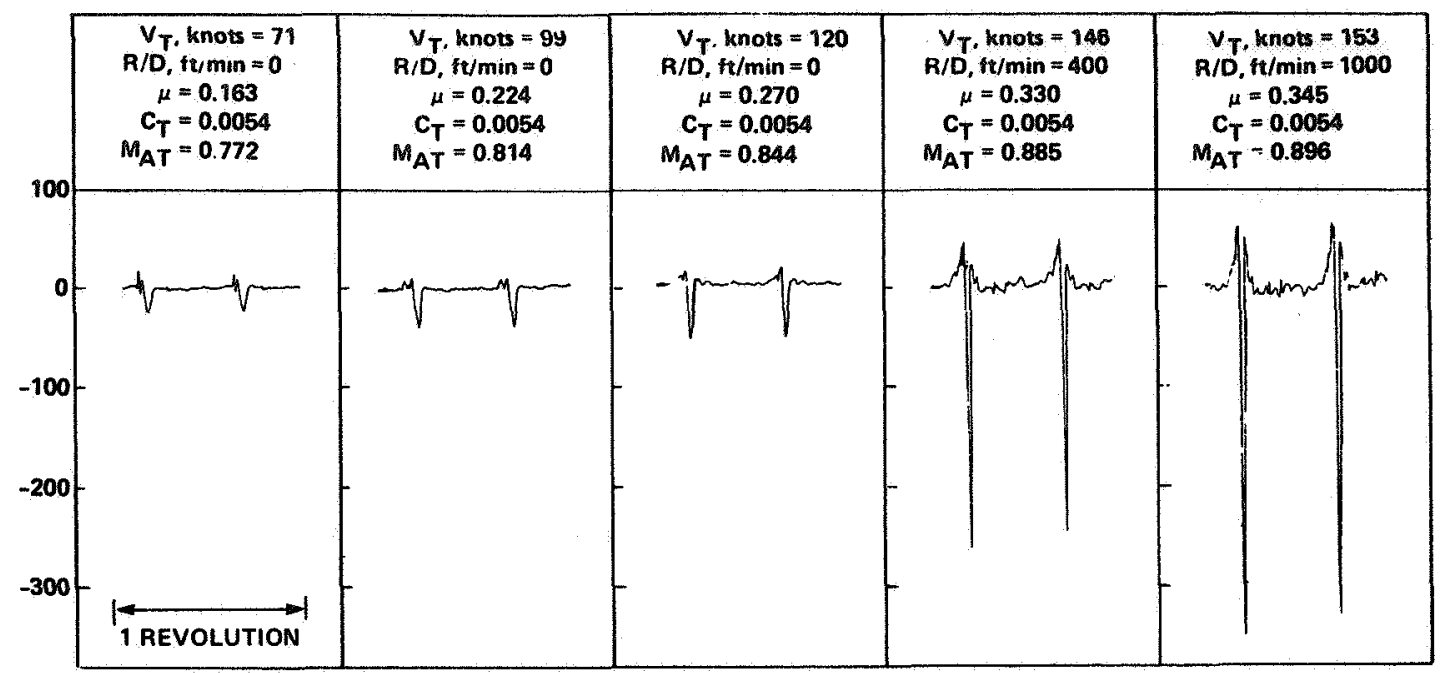
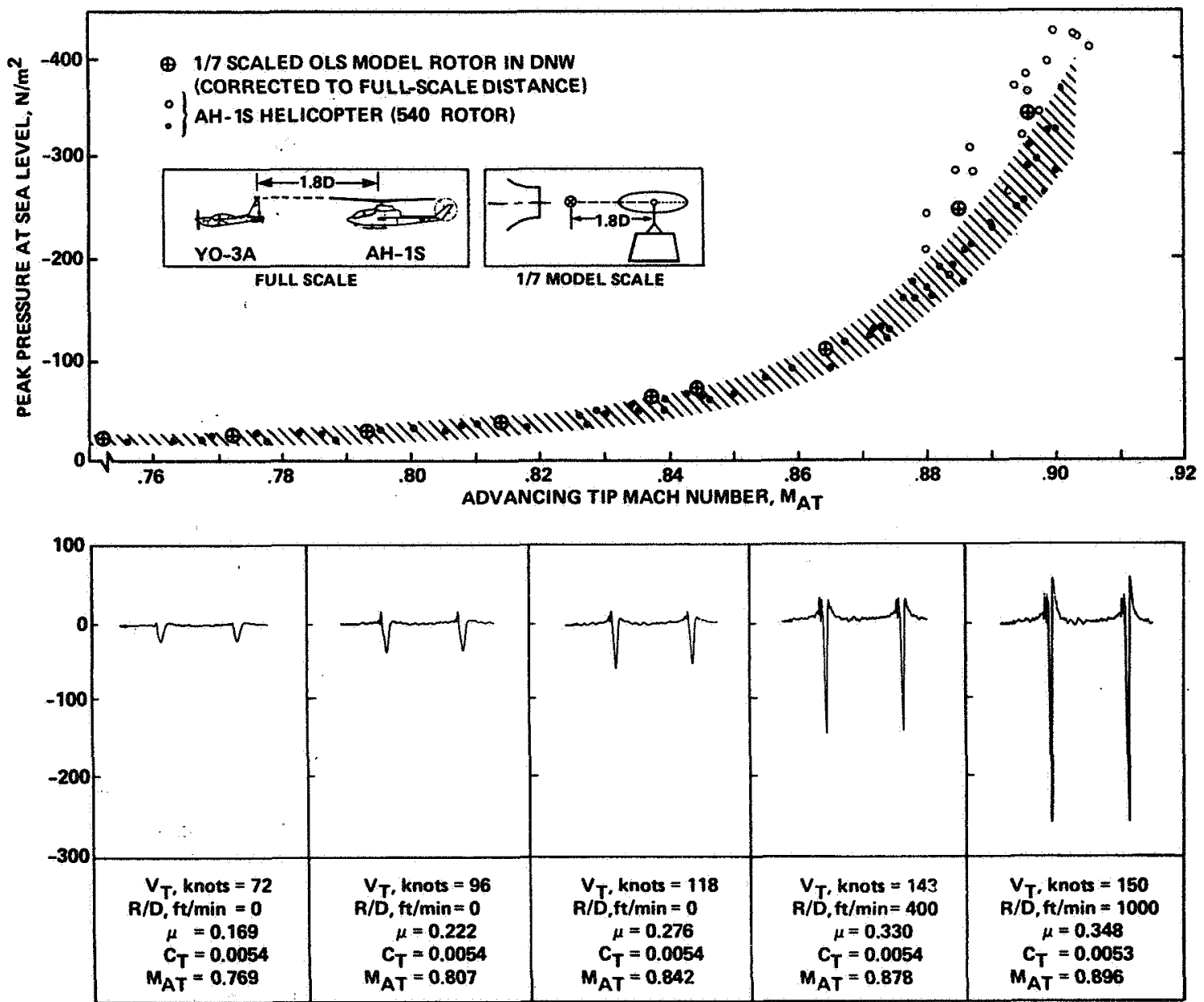

AH. $1 S$ (540 ROTOR) ACOUSTIC SIGNATURES

Figure 32.- Comparison of model and ful1-scale acoustic pressure for an in-plane microphone 1.8 rotor diameters ahead. 

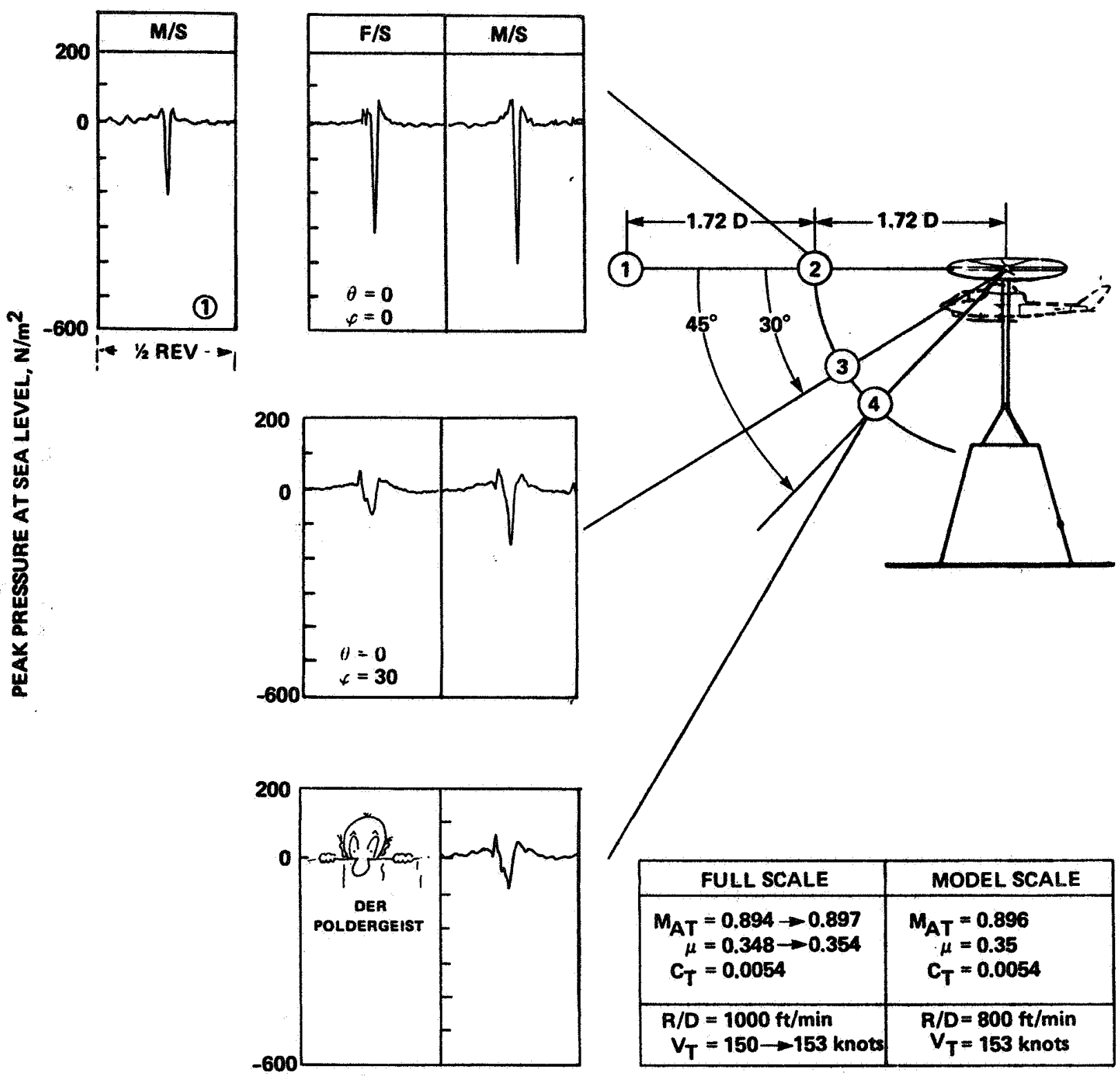

(a) Longitudinal directivity $\left(\mathrm{M}_{\mathrm{AT}}=0.896\right)$.

Figure 33.- Comparison of model and full-scale impulsive noise directivity. 
IN-PLANE NOISE SIGNATURES

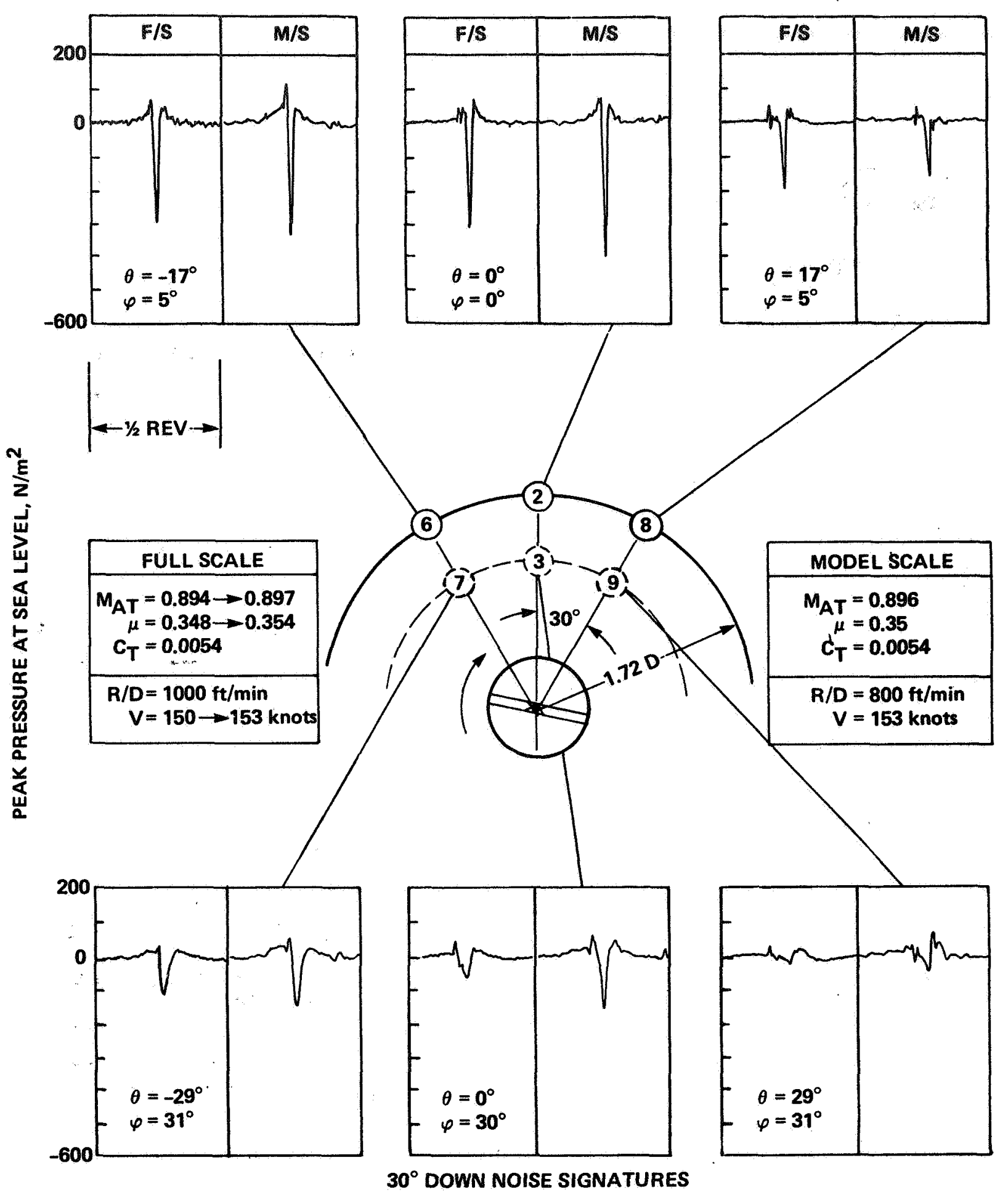

(b) Lateral directivity.

Figure 33.- Concluded. 


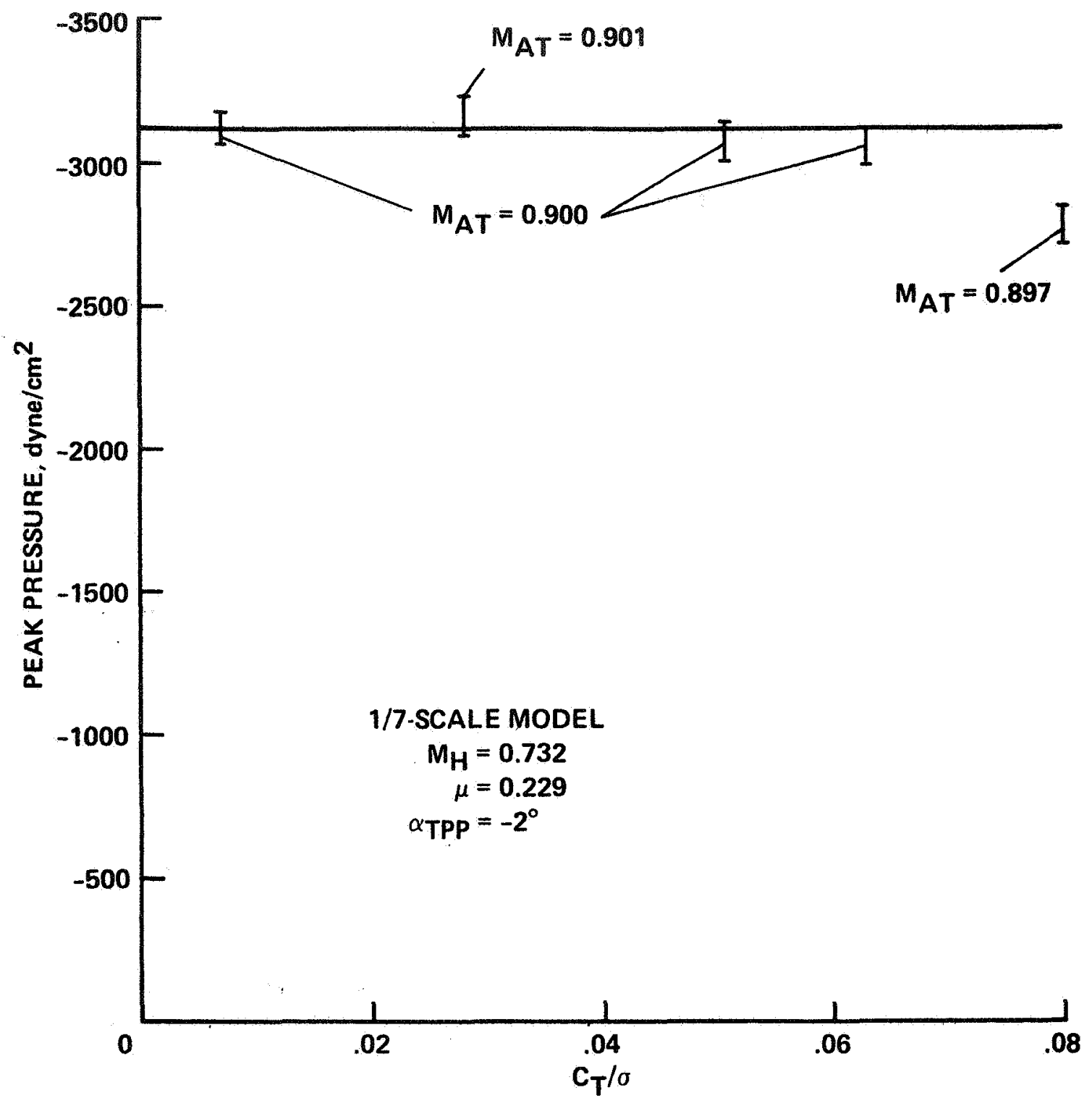

Figure 34.- Peak pressure versus changes in thrust coefficient (UH-1H model rotor).

The rapid increase in the magnitude of the negative-pressure pulse with increasing advancing-tip Mach number is reemphasized in figure 35. The solid curve was obtained at varying advance ratios $(0.091$ to 0.264$)$, and the dashed curve was obtained at a constant advance ratio (0.229). The similarity of the two curves over a range of advancing-tip Mach numbers $(0.87$ to 0.93$)$ shows that for high-speed impulsive noise, advancing-tip Mach number is the dominant parameter. Small changes can occur in advance ratio without significantly altering in-plane acoustic 


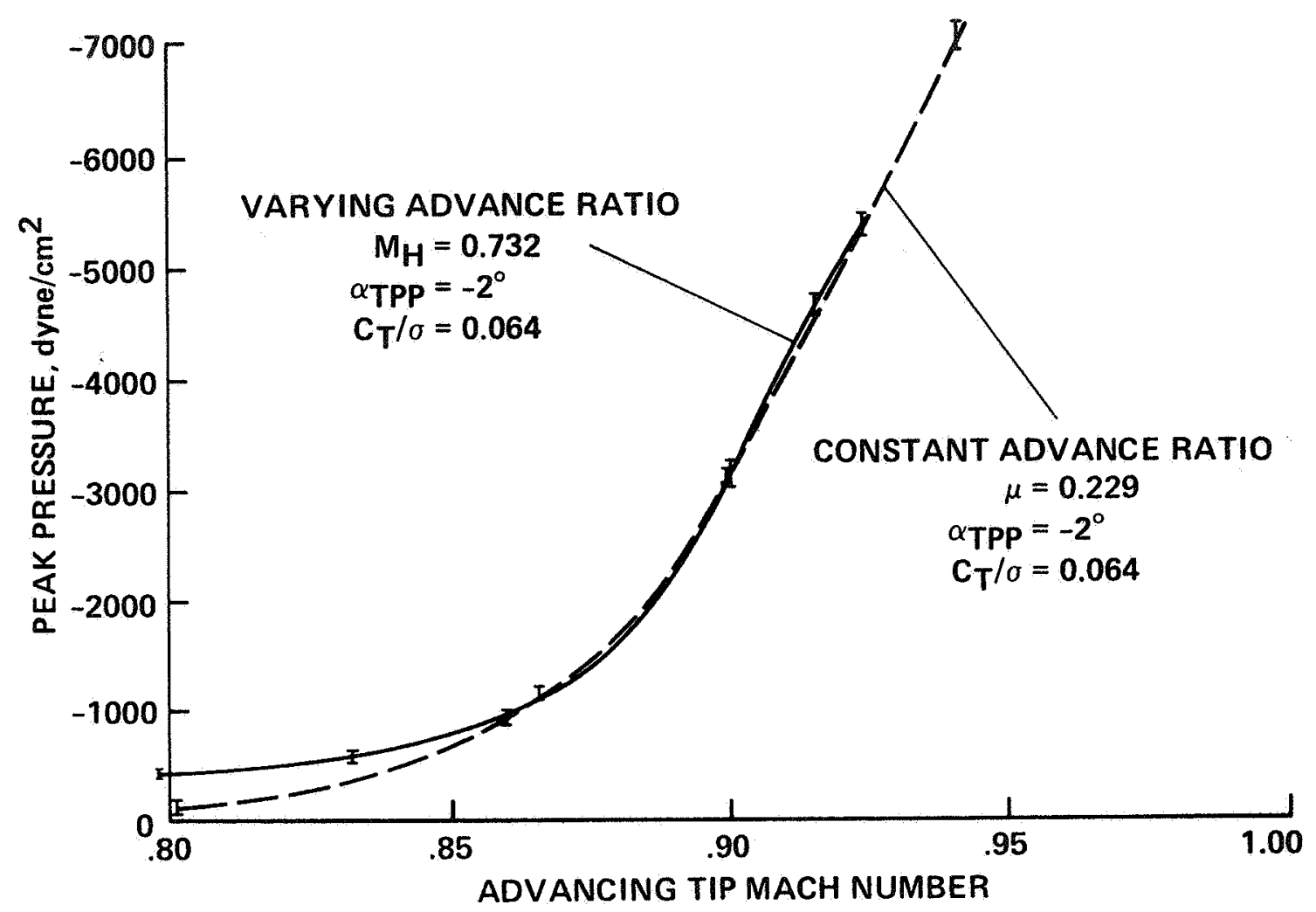

Figure 35.- Peak pressure versus advancing-tip Mach number: wind-tunnel data (UH-1H model rotor).

radiation, if advancing-tip Mach number is held constant. To helicopter designers, this means that small reductions in hover-tip speed or forward speed will significantly reduce impulsive noise through reductions in $\mathrm{M}_{\mathrm{AT}}$, but not through reductions in $\mu$.

Notice also that a notable difference in acoustic intensity does exist near $M_{A T}=0.8$ between the constant and varying advance-ratio cases. This difference is related to the radiation efficiency of steady and unsteady acoustic source mechanisms. At relatively high hover tip Mach numbers, $M_{H}=0.73$, and low advance ratios, $\mu=0.09$, each rotor blade experiences only small variations in the local Mach number around the azimuth, implying that the steady compressible source mechanisms are the most likely radiators of far-field acoustic energy. At the same $\mathrm{M}_{\mathrm{AT}}$, but at the lower hover-tip Mach numbers $\left(\mathrm{M}_{\mathrm{H}} \approx 0.65\right)$ and the higher advance ratios $(\mu=0.229)$, significant variations in the rotor-blade local Mach number occur and can be expected to exert more influence on the radiated acoustic field. In this case, unsteady acoustic-source mechanisms become more pronounced. The data shown indicate that the steady radiators of acoustic energy are more efficient generators of impulsive noise than the unsteady ones for similar advancing-tip Mach numbers. 
Perhaps the most interesting aspect of high-speed helicopter noise is the development of the saw-toothed waveform at high advancing-tip Mach numbers. This is shown in figure 36 together with a plot of the peak pressure versus advancing-tip Mach number $\left(_{H}\right.$ is constant and $\mu$ is varying) (ref. 13). In case $A\left(M_{A T}=0.867\right)$, a near-symmetrica1 pulse is observed; the subjective qualities could be described as a loud thumping. As the advancing-tip Mach number is increased, the symmetrical pulse becomes saw-tooth in character (case $B, M_{A T}=0.90$ ); the waveform consists of a large decrease in pressure followed by an extremely sharp increase in pressure $\left(\Delta \mathrm{P} / \Delta \mathrm{t} \cong 4 \times 10^{6}\right.$ dynes $\left./ \mathrm{cm}^{2} / \mathrm{sec}\right)$. Crispness (many harmonics) and intensity of the acoustic signature are its dominant features. At still higher advancing-tip Mach numbers (case $C, M_{A T}=0.925$ ), the peak negative pressure becomes very large, and the sudden rise in pressure becomes nearly instantaneous $\left(\Delta \mathrm{P} / \Delta \mathrm{t} \cong 10^{7}\right.$ dynes $\left./ \mathrm{cm}^{2} / \mathrm{sec}\right)$. Some overshoot can be seen, part of which is real and part of which is due to instrumentation bandwidth limitations. The noise generated by this latter waveform is rich in higher harmonics and can be subjectively classified as harsh and extremely intense.

Ft 'is known that this rapid increase in pressure (case $C$ ) is a radiating shock wave. Early indications of its formulation can be seen in case $B$ at the lower advancing-tip Mach number. Of course we know that local shock waves do exist near the tip of the rotor blade throughout this Mach number range. However, this acoustic plot suggests that these local shock waves "delocalize" at a certain "delocalization Mach number" and propagate to the acoustic far-field. Below the delocalization Mach number ( 0.9 for the NACA 0012 airfoil), all shock waves are confined to the blade. Above the delocalization Mach number, shock waves on the surface of the blade radiate as shock waves to the acoustic farfield (see refs. 20 and 22).

Although it is obvious that such processes as transonic shock generation and propagation are nonlinear, much can be learned by looking at the geometry of the 1 inear aerodynamic/acoustic process. Consider the space-fixed trajectory of a simple point source near the tip of a rotor blade. Its trajectory is the epicycloid pattern normally associated with the shed tip-vortex, as shown in figure 37 . If at regular azimuth angles $(\psi)$ a pulse is emitted in space and allowed to propagate at the ambient speed of sound, depicted as a circle (a sphere in three dimensions), these pulses form the crescent-shaped wave shown. In effect, disturbances are propagating away from a source moving at almost the ambient speed of sound. As a result, disturbances accumulate and cause the "Doppler amp1ification." As the advancing-tip Mach number increases (higher rpm or forward speed), the accumulation of disturbances becomes so great as to form local shocks and eventually the radiating shock wave. This accumulation process is represented as a singular integral, as we have seen in equations (1) and (2). The factor $1 /\left(1-M_{r}\right)$ is an integrable singularity for typical rotor geometry. 


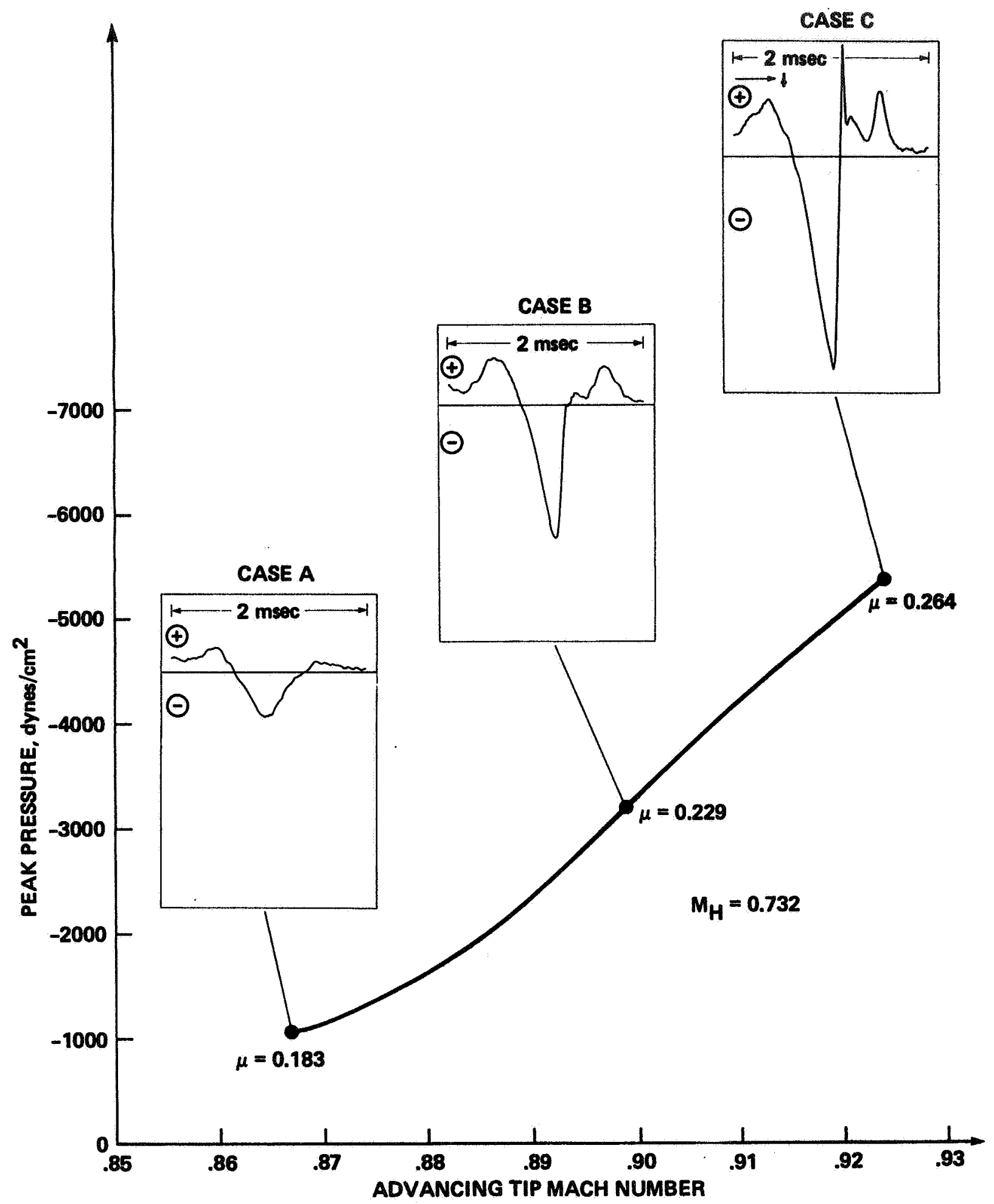

Figure 36.- Waveform shape versus advancing-tip Mach number. 


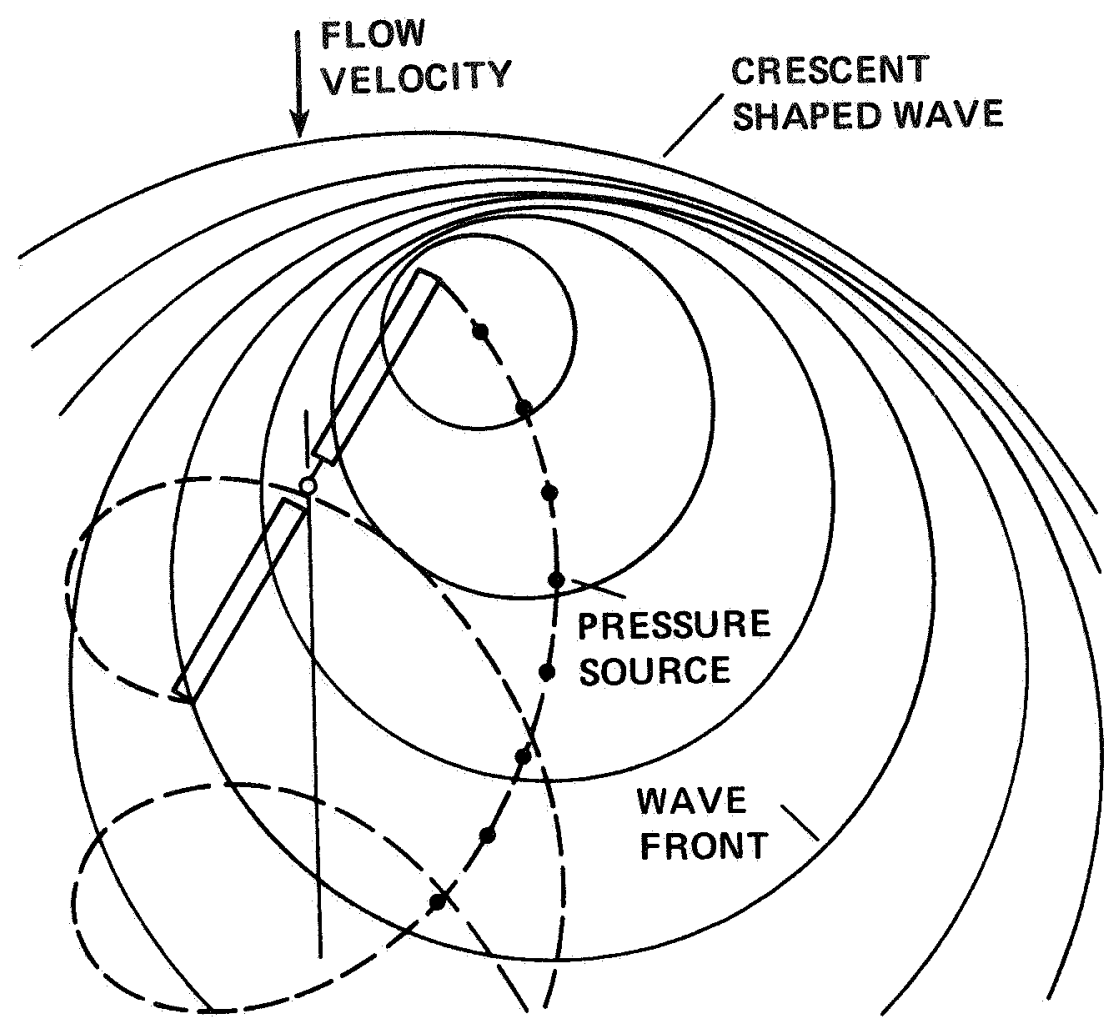

Figure 37.- Waves propagating from advancing blade (from ref. 9).

When these waves coalesce and a radiating shock wave develops, shadowgraph and Schlieren techniques can be used to visualize the radiation process. Using short-duration, high-intensity light pulses, the rotor's motion can be photographically frozen in space. A top view of the radiation process is shown in figure 38 for a rotor with an advancingtip Mach number of 0.92. These excellent photos were taken by J. Tangler of an approximately 2-ft diam model helicopter rotor (see ref. 9).

The top view of this small model-rotor experiment dramatically illustrates how local disturbances (in this case shock waves) are propagated to the observer in the far-field. At $\psi \approx 48^{\circ}$ (fig. 38), a smalldisturbance field, which is quite diffuse, is seen off the tip of the rotor. When $\psi=92^{\circ}$, the field has increased and moved slightly aft along the chord line. At $\psi \approx 151^{\circ}$, the waves are distinctly seen to move forward again and are traced into the region of $f$ the tip of the blade. Finally, at $\psi=159^{\circ}$, the waves move forward off the blade tip and escape to the acoustic far-field.

It should be noted that more than one wave appears to emanate from the tip of the rotor in this top view. It is believed that the two waves are really the extensions of the upper and lower surface shock systems on the blades. It is most likely that the stronger of the two radiating shocks is the second wave emanating from the tip of the rotor. Notice 


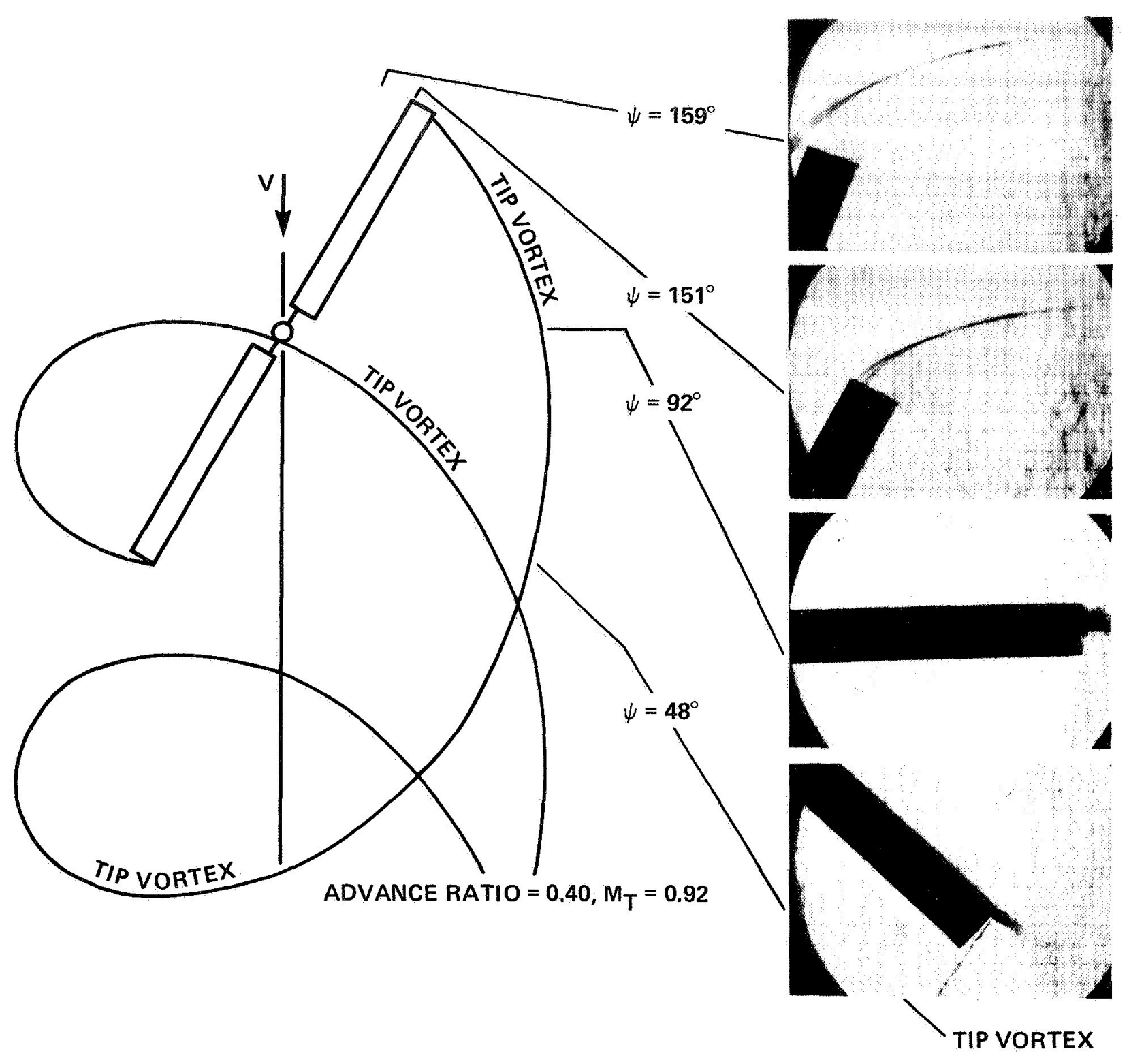

Figure 38.- High-speed advancing-blade shock formation (from ref.9).

also, however, that both waves coalesce into one wave as the disturbance propagates into the far-field. The additive nature of these radiating waves suggests the following explanation for the weak role of thrust changes on the measured in-plane acoustic disturbances. As thrust increases, the upper surface shock strength increases while the lower surface shock strength decreases. The net result is a near-constant acoustic level as the radiating waves coalesce far from the rotor tip. Some of the aerodynamic details of the tip flow fields themselves can be seen more clearly in two-dimensional in-plane views of the same threedimensiona1 event (ref. 13). 
Because most of the radiation process appears to be governed by Doppler amplification, it is logical to ask whether some of the key parameters of high-speed impulsive noise can be studied in hover. A "top view" of the linear acoustic wave behavior is sketched in figure 39 for a simple hovering impulse with a rotational Mach number of 0.9 .

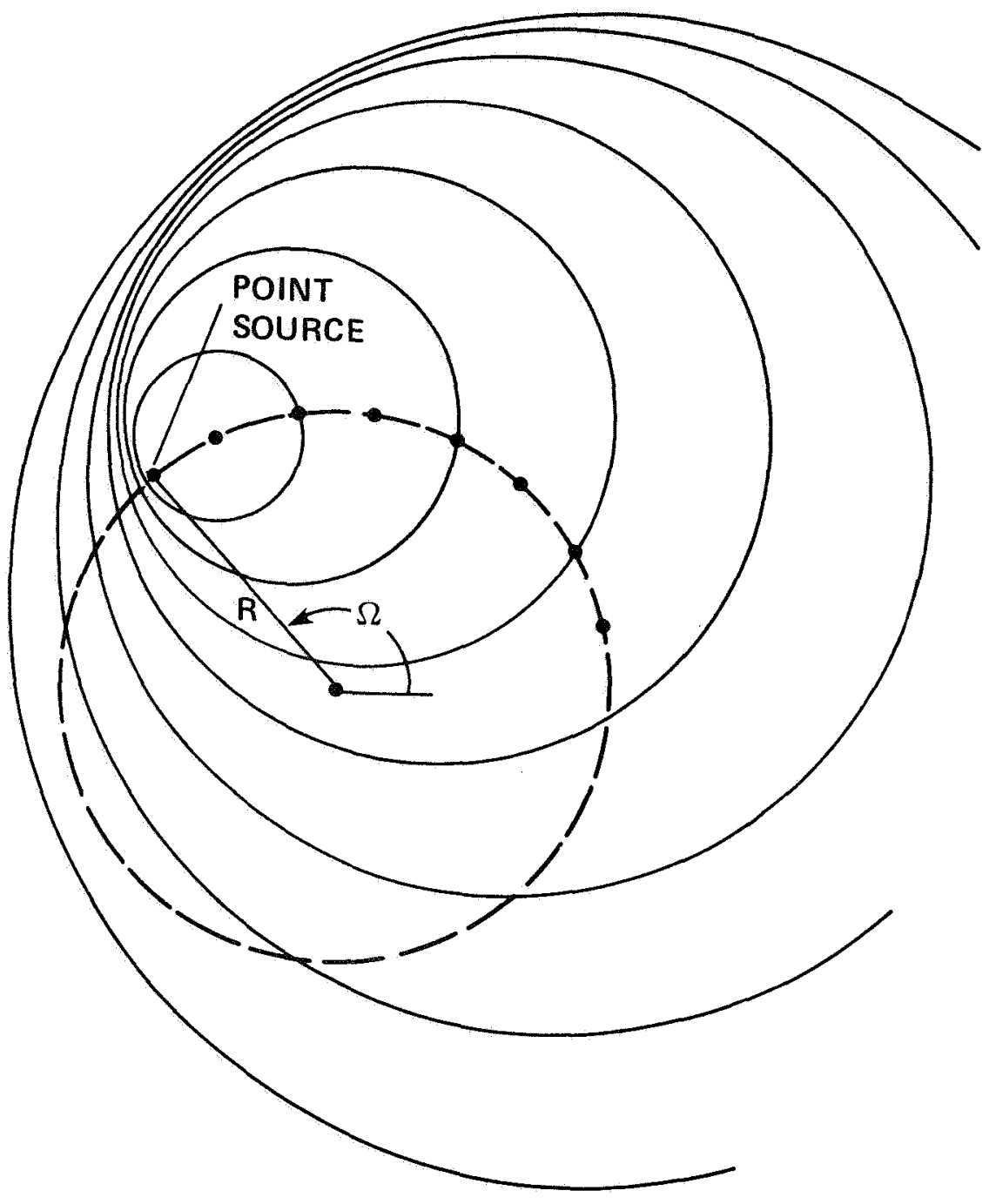

Figure 39.- Linear wave amplification (Doppler effects) of a rotating point source: $M_{T}=0.9$.

As seen from this sketch, a crescent shaped wave is formed which is quite similar to the one formed in the forward-flight testing. In the hovering environment, the acoustic radiation processes normally associated with high-speed impulsive noise can only be attributed to steady (when viewed on a rotor) aerodynamic events. Therefore, the mechanism of linear amplification can be studied without regard to the effects of 
periodic force or thickness variations normally encountered in highspeed helicopter forward flight.

To obtain data of sufficiently high quality, the high-speed hover acoustic data were taken in the Aeromechanics Laboratories' Anechoic Hover Chamber. The chamber was lined with polyurethane foam and designed to be anechoic (without acoustic reflections) down to $110 \mathrm{~Hz}$. As illustrated in figure 40, flow recirculation is avoided by allowing quiescent air to be drawn into the room through acoustically lined ducts, collecting the wake of the hovering rotor through an annular diffuser, and exhausting the wake to the outside. In its current configuration, the test chamber can accommodate rotors from 1.5 to $2.4 \mathrm{~m}$ in diameter.

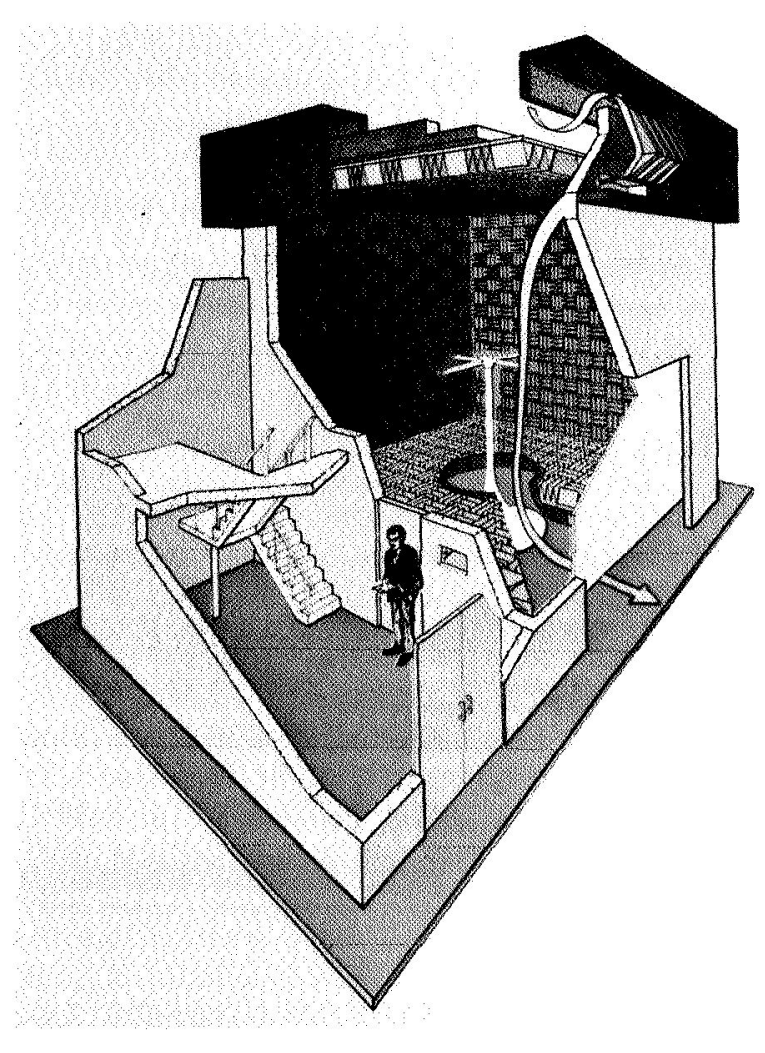

Figure 40.- Anechoic hover chamber.
One of the same rotors used in the scaling tests (a $1 / 7$ scale of a UH-1H main rotor) was run for these high-speed hover tests (ref. 15). The geometrically scaled rotor has a NACA 0012 airfoil section with a root-to-tip washout of $10.9^{\circ}$. Because thrust appeared to be unimportant in the high-speed noise generation process, a second set of untwisted but geometrically scaled rotor blades was run at near-zero net thrust. Some small positive net thrust was required to avoid shedwake interference effects. The data were taken with a microphone located within the tip-path-plane of the rotor at a distance of 1.5 rotor diameters $(r / D=1.5)$ from the hub. This in-plane microphone position is consistent with that used in previous in-flight and wind tunnel tests and is in a position to measure the most intense high-speed impulsive signature.

Figure 41 from reference 15 presents the measured acoustic signature at a hover tip Mach number $\left(\mathrm{M}_{\mathrm{T}}\right)$ of 0.8 . Two time scales are presented. Figure 41(a) depicts two blade passages, approximately one-half a complete rotor revolution. Figure $41(\mathrm{~b})$ is an expanded scale of the first acoustic pulse. The latter is used to emphasize the detailed waveform characteristics of the measured pulse. The most striking feature of the waveform at $M_{T}=0.8$ is its almost symmetrical character. This same character has been observed in full-scale and model-scale forward-flight testing at tip Mach numbers below the delocalization Mach number. 
$r / D=1.5, M_{T}=0.8$

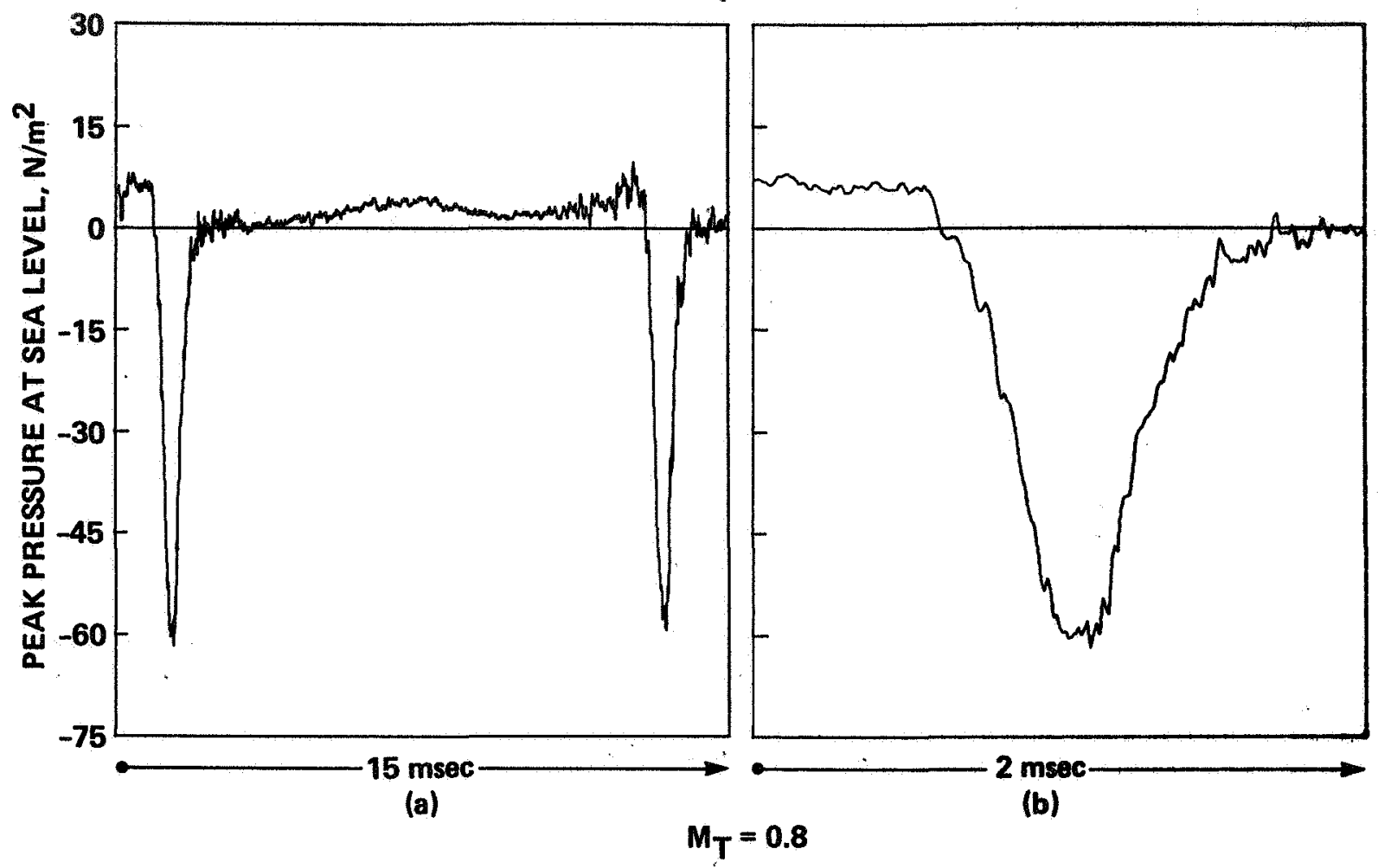

Figure 41.- UH-1H model blade acoustic pressure time-history, In-plane, $\mathrm{r}_{\mathrm{H}} / \mathrm{D}=1.5, \mathrm{M}_{\mathrm{T}}=0.8$.

Figure 42 illustrates the pressure time-history at a hovering tip Mach number of 0.9 . The peak negative-pressure amplitude of the measured pulse has increased dramatically and the pulse shape has now lost its symmetry. The resulting saw-toothed waveform is known to generate large amounts of high-intensity, higher-frequency noise. Again, this same type of waveform was measured on the same rotor system operating in forward flight at an advancing-tip Mach number of 0.9. In this prevlous test, Schlieren photographs were used to correlate the discontinuous increase in pressure with a radiating shock wave. It is apparent that a similar phenomenon is occurring in this controlled hover test.

At a hover tip Mach number of 0.962 (fig. 43), the saw-toothed pulse shape is firmly established and the negative pressure peak level has doubled from the $M_{T}=0.9$ condition. The large, discontinuous rise in pressure resulting from a radiating shock wave exhibits some variability from blade to blade. One particularly interesting aspect of the waveform shown in figure $43(\mathrm{~b})$ is the pulse width. At lower hover tip Mach numbers, the pulse width was observed to narrow with increasing rotor-tip speed up to the point of waveform transition from symmetrical to saw-tooth. Above this transition point, for example at $M_{T}=0.962$, the pulse width has become larger. Figure 43 also shows that a positive-pressure wave (bow 
$r / D=1.5, M_{T}=0.9$

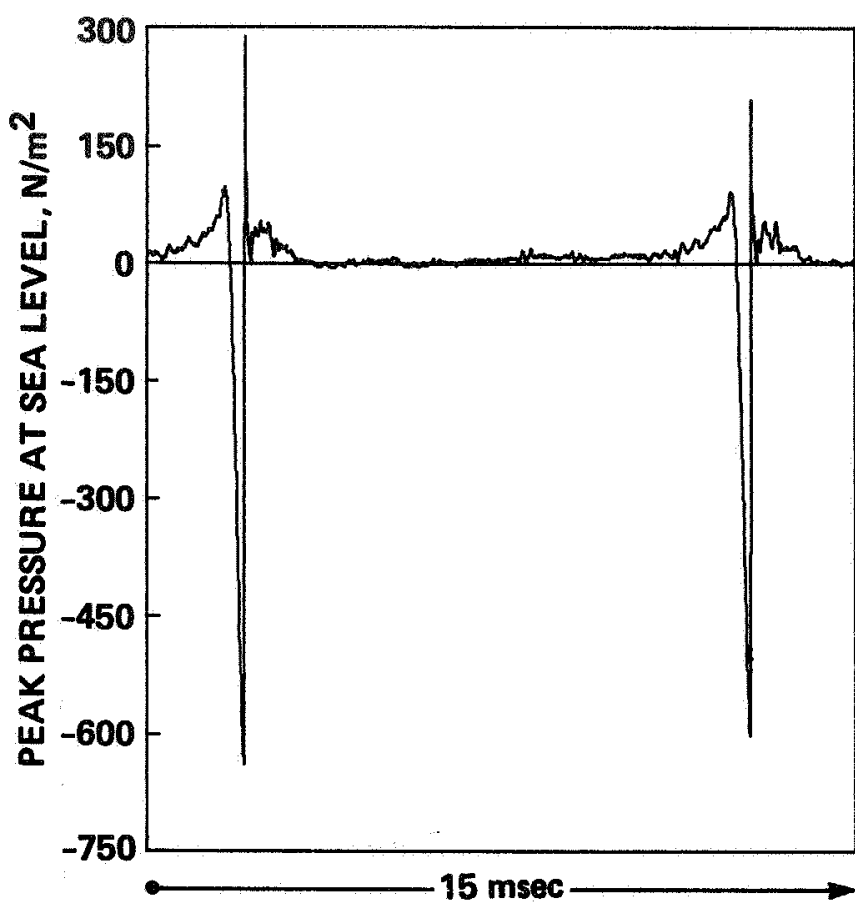

(a)

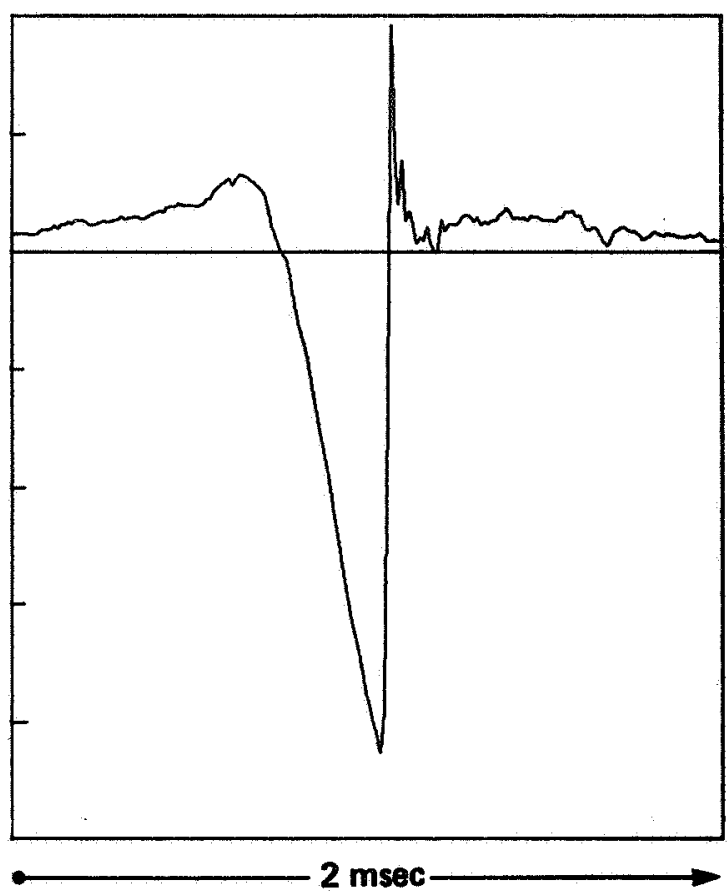

(b)

$$
M_{T}=0.9
$$

Figure 42.- Acoustic pressure time-history, in plane, $r_{H} / D=1.5$,

$$
\mathrm{M}_{\mathrm{T}}=0.9 \text {. }
$$

wave) begins to form. At still higher tip Mach numbers, the classical $\mathrm{N}$-wave of sonic boom research is likely.

In addition to the general increase of peak negative-pressure leve1 with increasing hover tip Mach number, the waveform transition from symmetrical to saw-tooth dominates the changing acoustic signature. Figure 44 illustrates the development of the radiating waveform discontinuity as measured in hover at 1.5 diam away. The sequence of waveforms in figure 44 shows that transition occurs over a very sma11 range in hover tip Mach number from 0.88 to 0.90 , with $\mathrm{M}_{\mathrm{T}}=0.89$ being the point of transition for the test rotor. Transition was found to be characterized by a simultaneous increase in peak negative-pressure level and the following rapid pressure rise. Both events were observed to be highly unsteady, even under controlled rotor test conditions.

It is also instructive to compare the peak negative-pressure amplitude of the measured waveform versus hover tip Mach number (fig. 45). A very rapid increase in level is noticed as $\mathrm{M}_{\mathrm{T}}$ approaches 0.9 . However, as $\mathrm{M}_{\mathrm{T}}$ increases beyond 0.9 to $\mathrm{M}_{\mathrm{T}}=1.0$, the increase in peak level is not as rapid, or the rate of increase of this peak negative-pressure level with Mach number becomes smaller. As noted in figure 45, the 


$$
r / D=1.5, M_{T}=0.962
$$

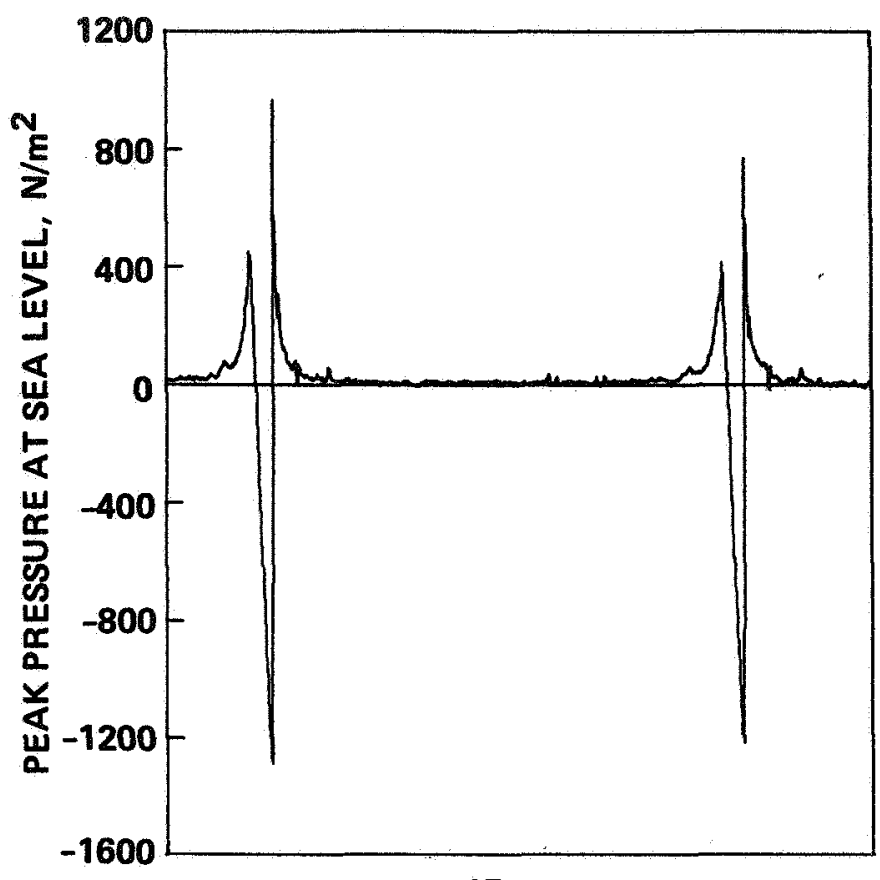

(a)

$$
M_{\mathrm{T}}=0.962
$$

Figure 43.- Acoustic pressure time-history, in-plane, $r_{H} / D=1.5$, $M_{\mathrm{T}}=0.962$.

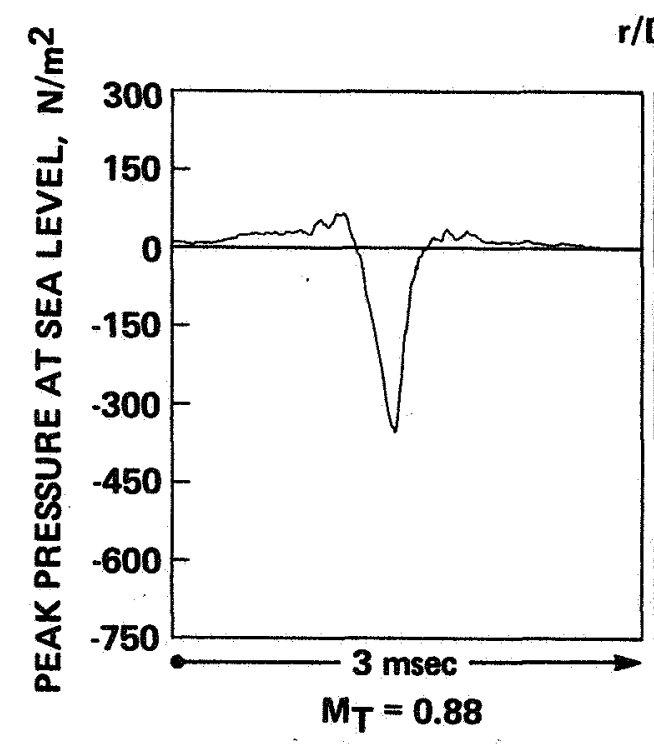

(a)

$$
r / D=1.5, M_{T}=0.88 \rightarrow 0.90
$$

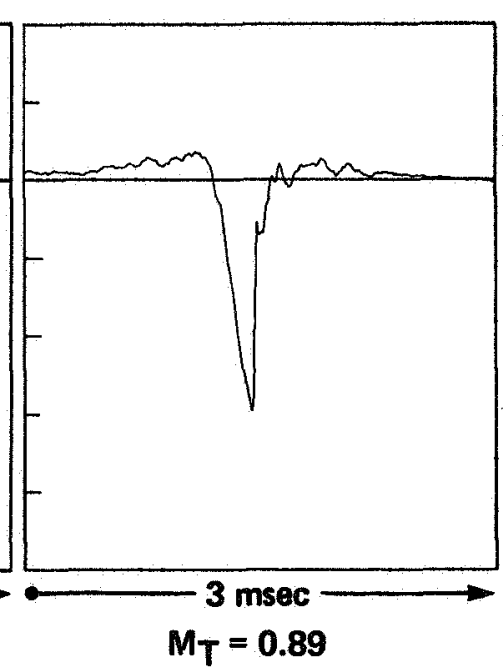

(b)

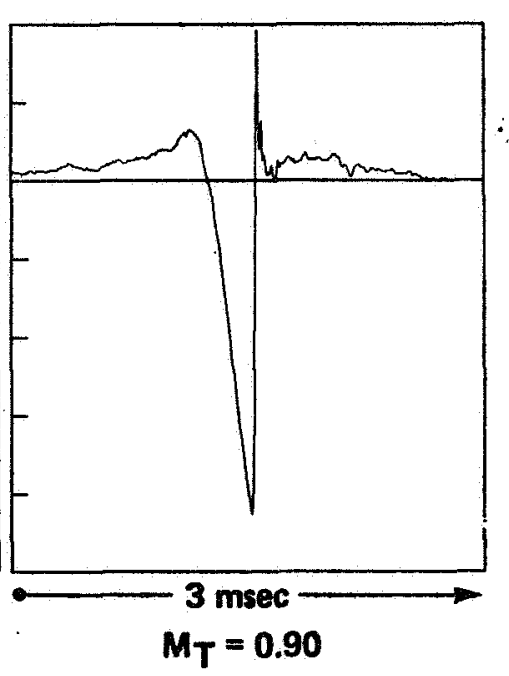

(c)

Figure 44.- Waveform transition; the development of a radiating discontinuity, in-plane, $\mathrm{r}_{\mathrm{H}} / \mathrm{D}=1.5, \mathrm{M}_{\mathrm{T}}=0.90$. 


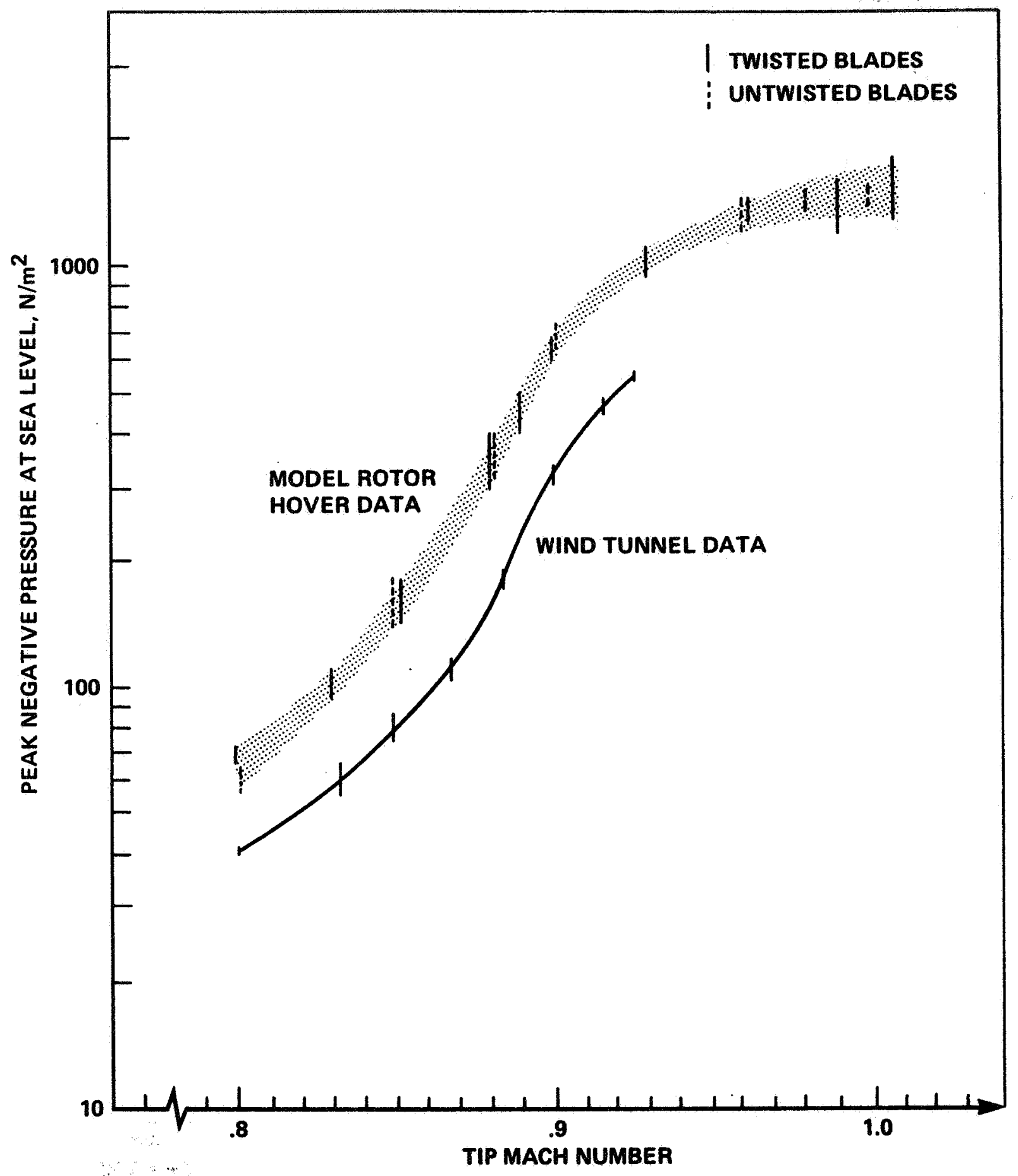

Figure 45.- Peak negative-pressure level versus tip Mach number for rotor in hover and forward flight, in-plane, $r_{H} / D=1.5$. 
shaded area depicts the degree of unsteadiness in the measured data. The vertical solid bars reflect data taken with the UH-1H model twisted blades, and the dashed vertical bars are for the same dimension mode1 rotor using untwisted blades. The correlation between twisted and untwisted results is good. This result gives further support to the idea that at the in-plane microphone position, rotor thrust is not a primary acoustic parameter.

The most remarkable observation about the hovering rotor data is their similarity to the model-scale acoustic data taken in forward flight. Although the amplitude of the hovering data is larger than that of the wind-tunnel data, its shape and character are remarkably similar. As in forward flight, the wave changes character at a tip Mach number of 0.9 - radiating shock-like disturbances to the acoustic far-field. The major mechanisms of the high-speed impulsive-noise phenomenon are present in this simple hovering rotor experiment. This fact will become more and more evident as the discussion proceeds.

It is natural to question the accuracy of the mode1-rotor experiment. A major concern is the accurate measurement of the rotational tip Mach number. Fortunately, the experiment has been repeated many times in at least three different facilities throughout the world. More recently, humidity effects on Mach number have been accounted for. The results shown here have been consistent and reproducible within about a $\pm 10 \%$ error in absolute amplitude. A1l delocalization Mach-number trends are also reproducible to within 0.005 of a Mach number.

The simple hovering rotor is a natural place to begin to compare acoustic theory with experiment. Experimentally, it has been shown that thrust plays a small role (if any) in the in-plane noise radiation. If al1 nonlinear effects are also neglected $\left(T_{i j}=0\right)$, we can calculate the linear thickness noise equating the first two terms of equation (1) to zero and solving the resulting integral equation:

$$
P^{\prime}(\vec{x}, t)=\frac{\partial}{\partial t} \iint\left[\frac{\rho_{0} v_{n}}{\left.r \mid 1-M_{r}\right]}\right]_{\tau} d S(\vec{n})
$$

Fortunately, the entire right-hand side of this equation is known. The major complications are the correct handling of the retarded time which is complicated by the geometry of the rotor problem (ref. 8). However, the tip Mach number of normal helicopter rotors is less than 1.0 , facilitating a simple and straightforward calculation of equation (10).

The numerator of the right-hand side of equation (10) represents the equivalent acoustic sources (monopoles) that are created because each segment of the finite-thick rotor blade must displace mass as it moves through the medium. Thus, the portions of the blade that push fluid away from the blade are represented as acoustic monopole sources; those that flow over a two-dimensional airfoil section are given in figure 46 . From 


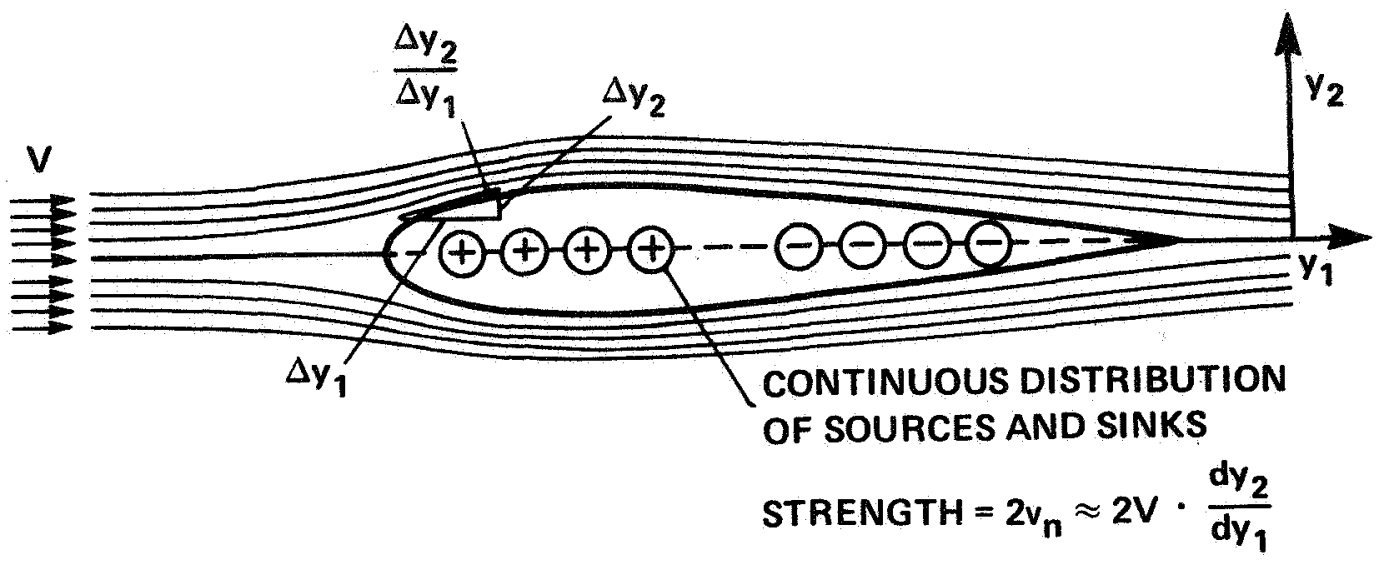

Figure 46.- Monopole acoustic sources.

sma11-perturbation theory for a slender two-dimensional body, the normal component of surface velocity is expressed as follows:

$$
\mathrm{v}_{\mathrm{n}} \approx \mathrm{V} \cdot(\text { local surface slope) }
$$

where $V$ is the local free-stream velocity of the blade element ( $\Omega R$. for the hovering rotor).

As shown in the simple geometrical patterns of a hovering rotor (fig. 39), exactly how the acoustic waves are collected in space is an important part of this linear acoustic problem. Correct treatment of the retarded time is essential, for it governs the amplification factor, $1 /\left(1-M_{r}\right)$ of equation (10), as well as keeping track of when the wave propagation from the singularities in motion actually reach the observer.

Consider a simple hovering point singularity (top view) which emits a disturbance at position "P" that travels at the ambient speed of sound in the medium and arrives at the observer's position " 0 " at some time later, $r / a_{0}$ (fig. 47). The velocity of the point "P" at any instant of time is $\Omega R$, and $\mathrm{M}_{r}$ is the component of that velocity vector along the $r$ direction divided by the speed of sound. From the given geometry, observer time is related to emission time $\tau$ by the implicit relationship

$$
\tau=t-\frac{r(t, \tau)}{a_{0}}
$$

where

$$
\mathbf{r}^{2}=\left(\mathrm{r}_{\mathrm{H}}+\mathrm{R} \cos \psi\right)^{2}+(\mathrm{R} \sin \psi)^{2}
$$




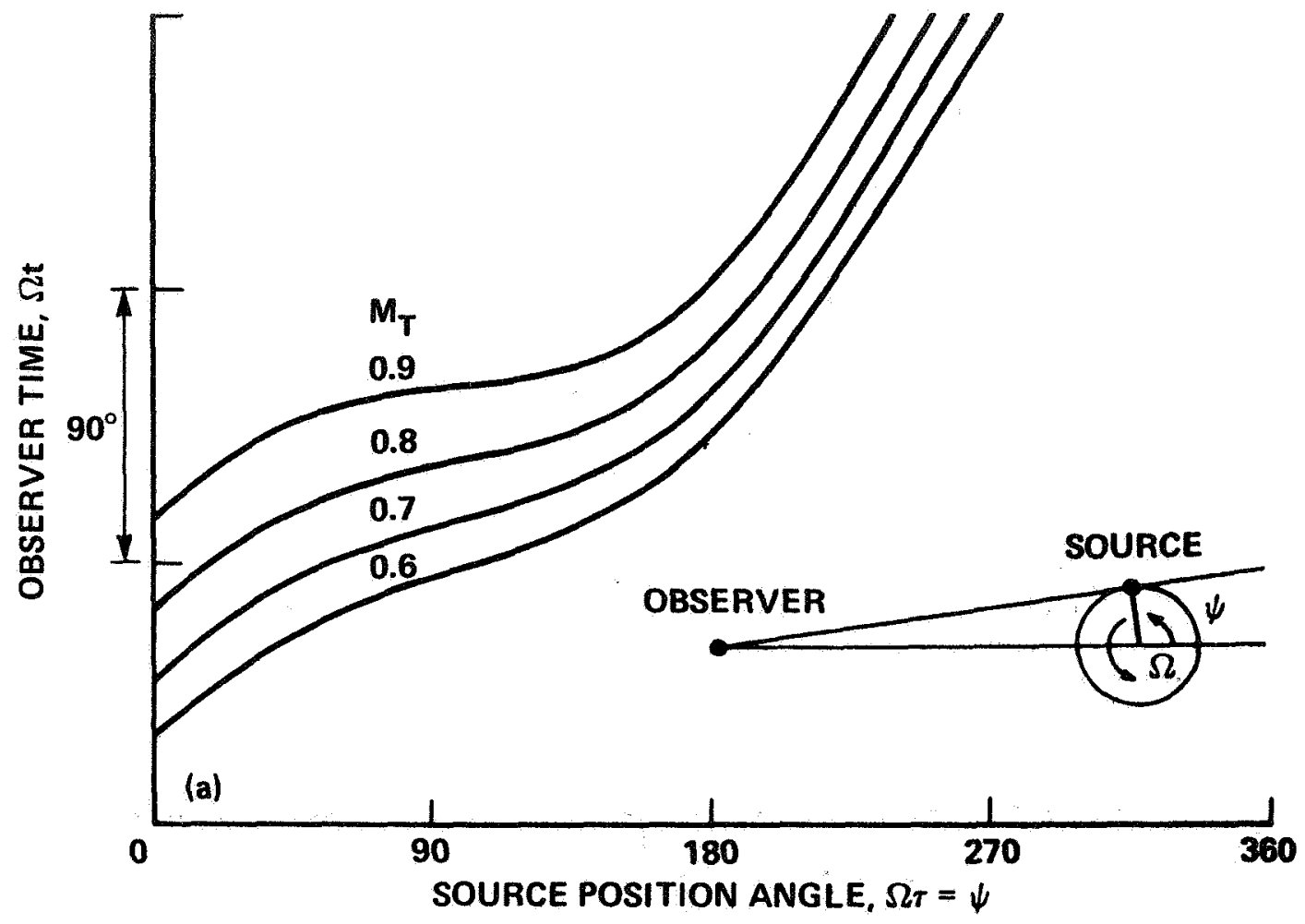

Figure 47.- Retarded time factor.

Therefore, a point source which was emitted at the retarded time $\tau$ takes time $r(t, \tau) / a_{o}$ to travel the distance $r$ arriving at time $t$. If the rotor is rotating at angular velocity $\Omega$, then pulses that are emitted at selected azimuthal positions, $\psi(\psi=\Omega \tau)$ obey the expression

$$
\begin{aligned}
& \Omega \tau=\psi=\Omega t-\frac{\Omega r(t, \tau)}{a_{0}} \\
& \frac{d \tau}{d t}=\frac{1}{I-M_{r}}
\end{aligned}
$$

which is plotted in figure 47 . Figure 47 shows that as the Mach number approaches 1, a large region of blade azimuth contributes to a narrow pulse width. The result is an inherent amplification of local source effects by the factor $1 /\left(1-M_{r}\right)$ which is illustrated in figure 48 .

Part of this amplification of acoustic energy is explicitly accounted for in equation (10). The Doppler factor, $1 /\left(1-M_{r}\right)$, which is the Jacobian of a coordinate transformation, already appears in the acoustic source term and represents the formation of a velocity potential wave with respect to the medium. A second Doppler factor is implicit in 


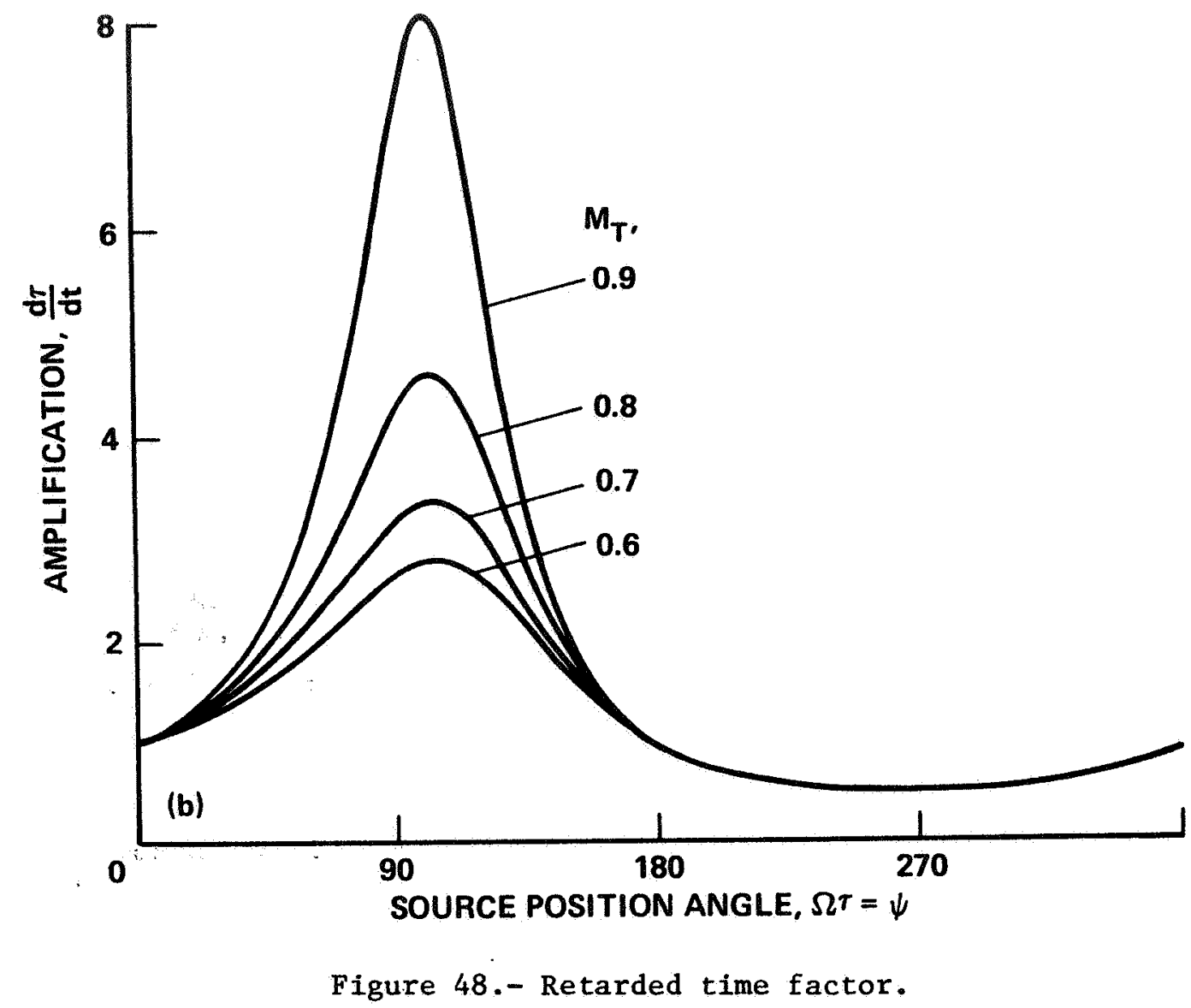

equation (10) and does not enter until the time-derivative of the potential function is taken. In each case, large-amplification effects are evident as advancing-tip Mach numbers approach 1 . These simple expressions show that the blade tip is acoustically the most efficient portion of the rotor, with acoustic efficiency decaying rapidly for inboard radial positions. It can also be concluded from figure 48 that the dominant azimuthal source position is near the tangency point of a straight line drawn from the observer to the circle described by the tip of the rotor. At this azimuthal position, all line sources along the rotor blade arrive at the observer position at approximately the same time.

The final evaluation of the integral of the monopole sources is performed by dividing the rotor blade into chordwise and spanwise elements and by summing each contribution according to the geometric rules previously discussed. Finally, a time differential is performed yielding the acoustic pressure at the observer location. Therefore, equation (10) becomes 


$$
P^{\prime}(x, t) \approx 2 \frac{\partial}{\partial t} \sum_{k=1}^{\ell} \sum_{i=1}^{n} \rho_{\circ}\left[\frac{V\left(d y_{2} / d y_{1}\right)_{k i}}{r\left|1-M_{r}\right|}\right] d y_{1_{i}} d y_{3_{k}}
$$

Additional details describing these computational procedures can be found in reference 8 .

A comparison between linear thickness calculations for hover versus the experimental data previously presented is shown in figures 49-52 for several different hover tip Mach numbers (ref. 15). The striking features of the comparison between theory and experiment in hover at $\mathrm{M}_{\mathrm{T}} \cong 0.8$ (fig. 49) are the similarity in pulse shape and the discrepancy in peak pressure levels. As in forward flight at advancing-tip Mach numbers below 0.9 , thickness-noise theory misses the measured negativepressure peak levels by a factor of about 2 .

The comparison of theory and experiment as $\mathrm{M}_{\mathrm{T}}$ is increased to 0.88 ( $\mathrm{fig}$. 50) remains similar to that made at $\mathrm{M}_{\mathrm{T}}=0.8$. The waveform shape is still generally symmetrical but the peak negative-pressure leve 1 is underpredicted by slightly more than a factor of 2 . As was noted previously, $\mathrm{M}_{\mathrm{T}}=0.88$ is slightly less than the delocalization Mach number for waveform transition, at least as measured at $\mathbf{r}_{\mathbf{H}}=1.5$ with the test rotor.

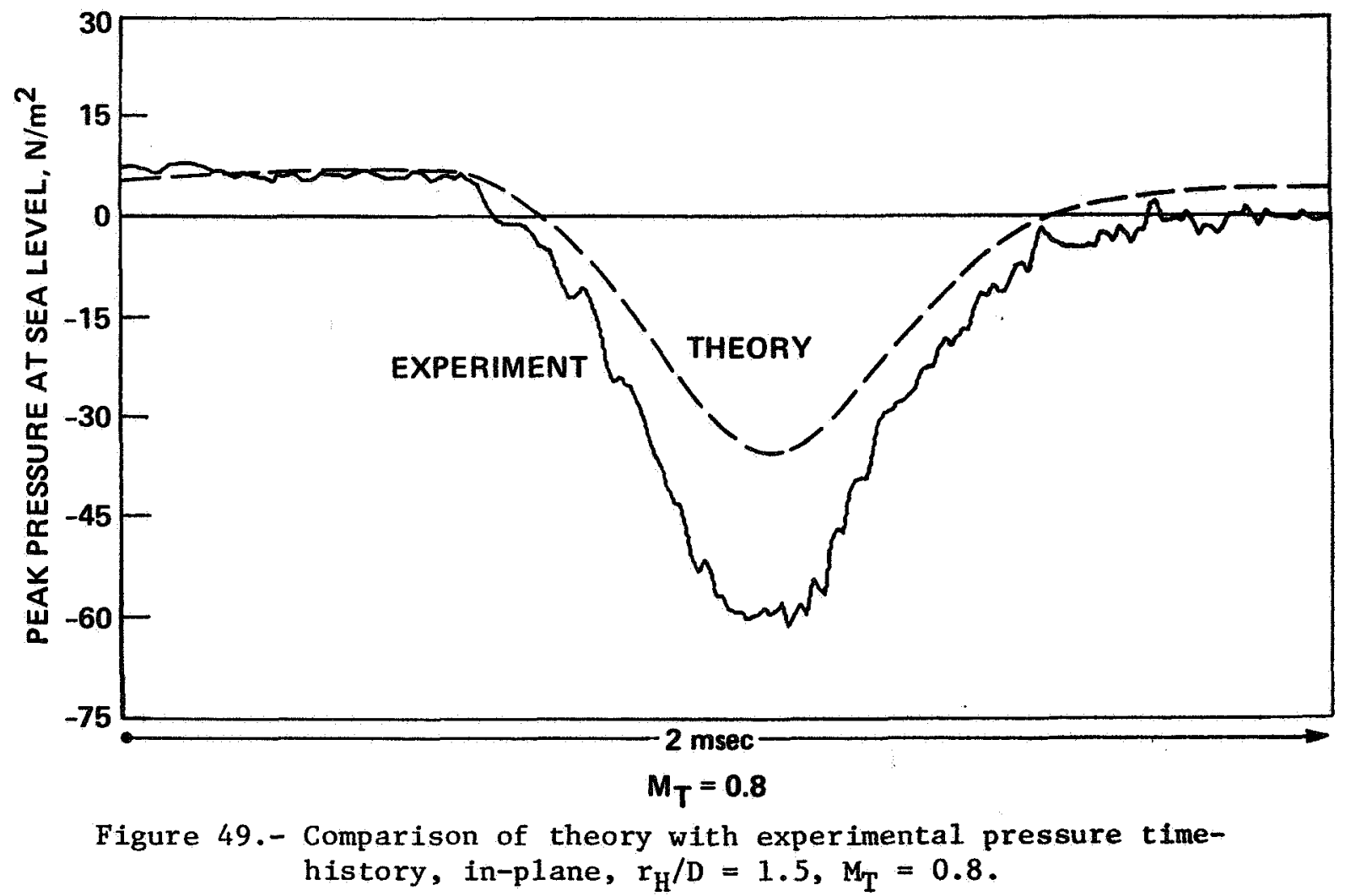




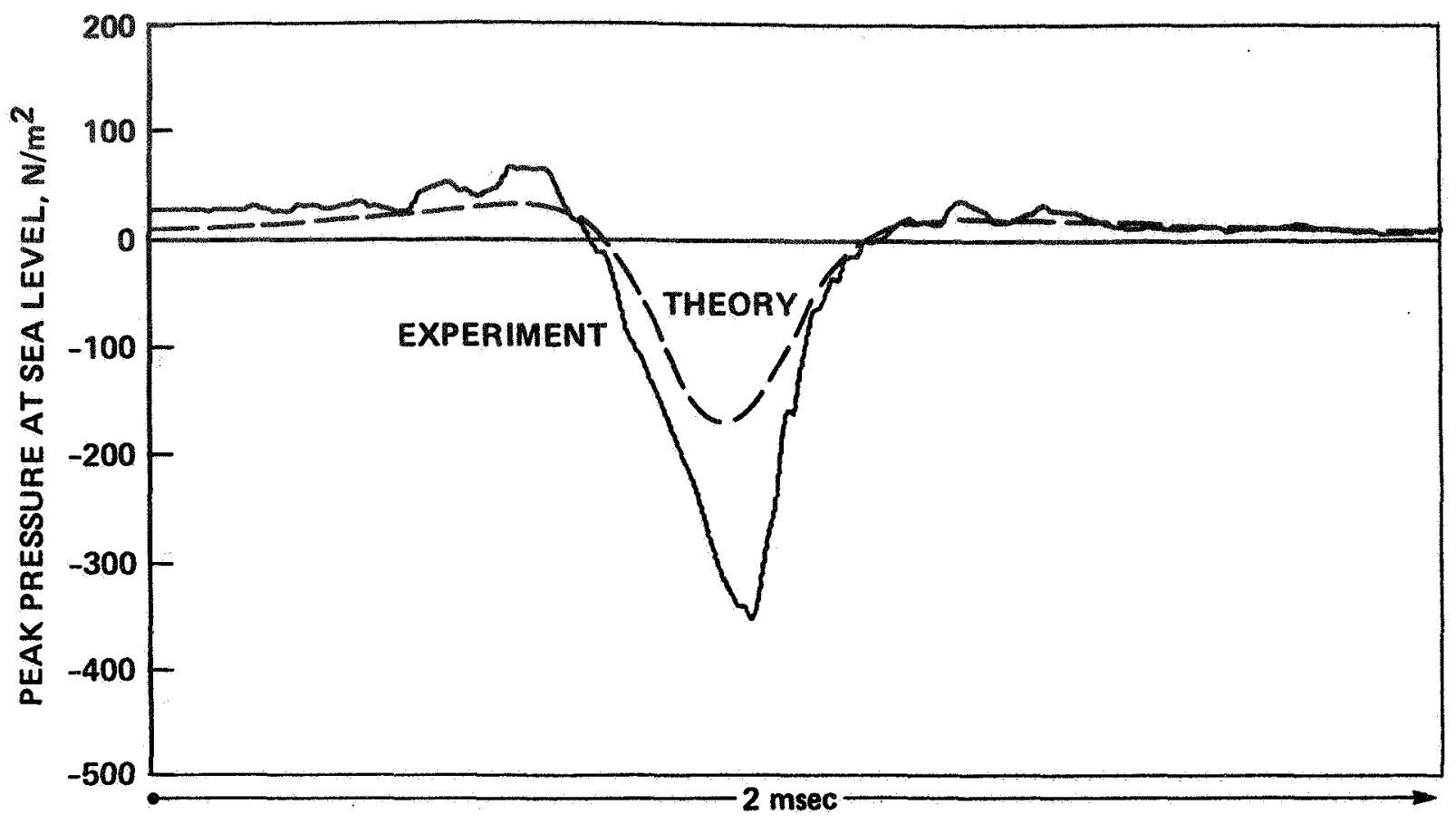

Figure 50.- Comparison of theory with experimental pressure timehistory, in-plane, $r_{\psi} / D=1.5, M_{T}=0.88$.

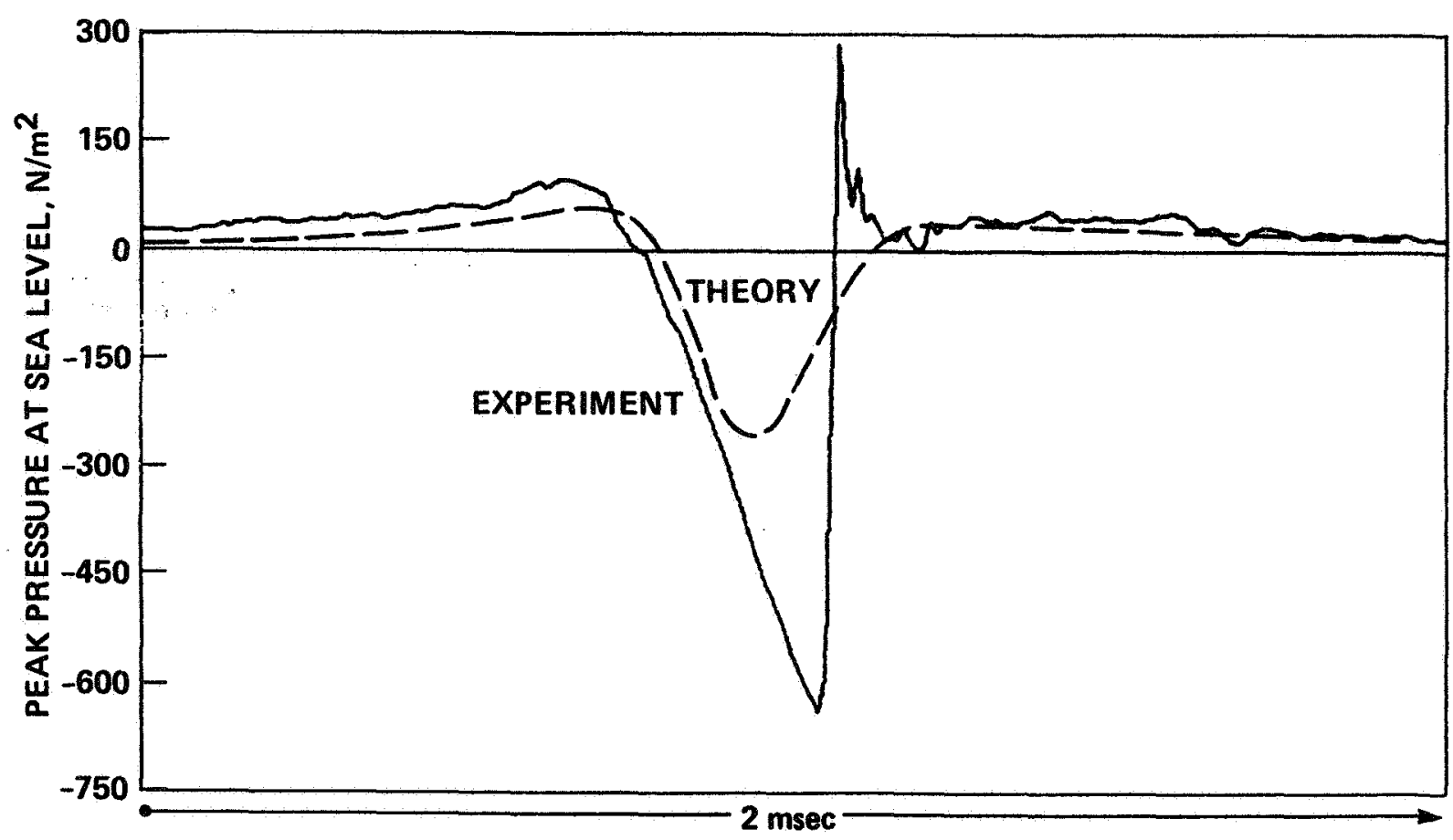

Figure 51.- Comparison of theory with experimental pressure timehistory, in-plane, $\mathrm{r}_{\mathrm{H}} / \mathrm{D}=1.5, \mathrm{M}_{\mathrm{T}}=0.9$. 


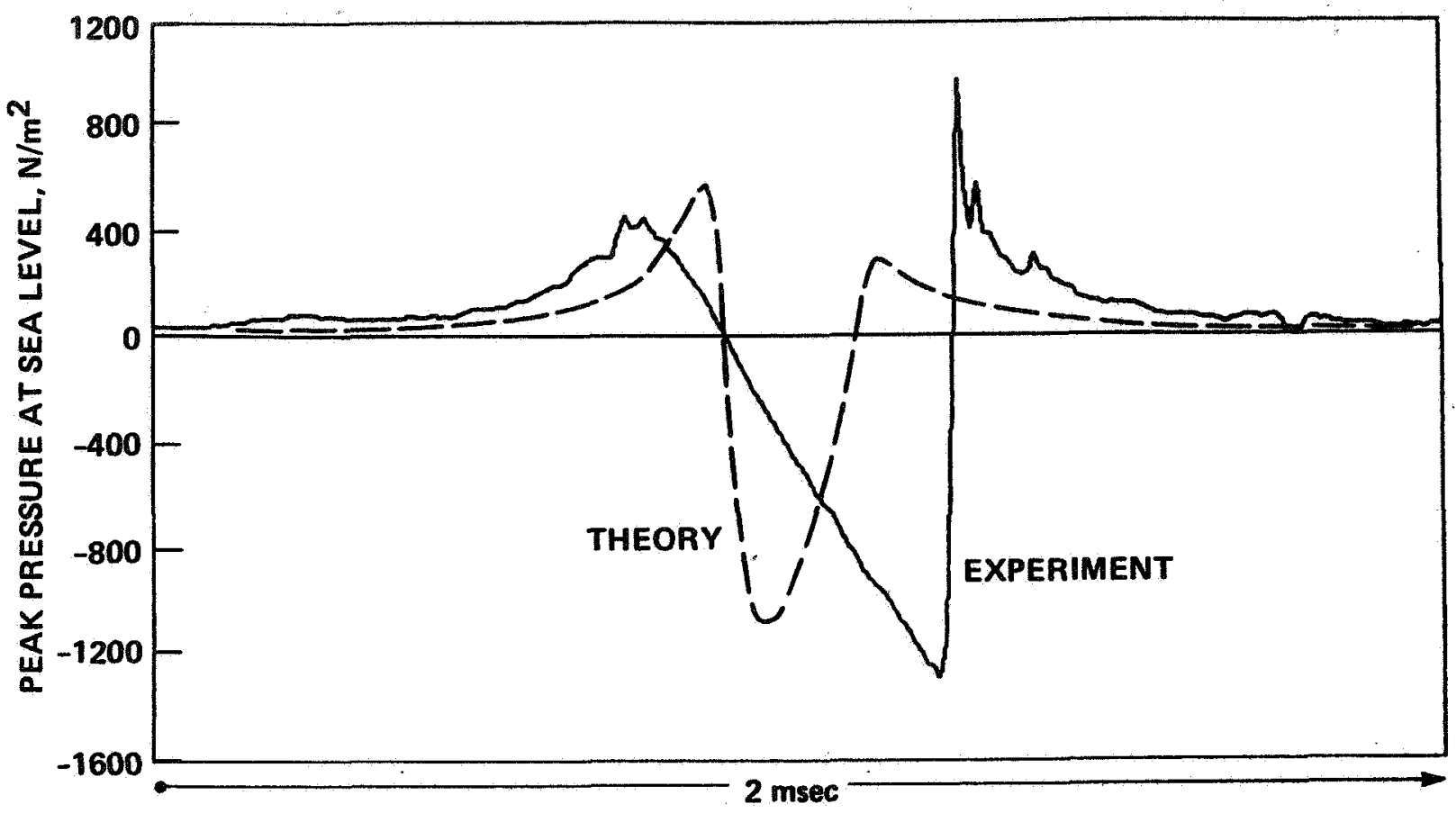

Figure 52.- Comparison of theory and experimental pressure time$\mathrm{h}$ istory, in-plane, $\mathrm{r}_{\mathrm{H}} / \mathrm{D}=1.5, \mathrm{M}_{\mathrm{T}}=0.962$.

At hover tip Mach number of 0.9 , the situation becomes even worse (fig. 51). The amplitude of the peak negative-pressure pulse is again underpredicted, by a factor of about 2. However, as indicated previously, there is also a dramatic change in the waveform of the experimental data that is not predicted by the linear theory.

The comparison becomes even more intriguing at a hover tip Mach number of 0.962 (fig. 52). The theoretical waveform is still symmetrical and generally smooth in shape and thus does not compare favorably with the measured data. In addition, theory now only slightly underpredicts the peak negative-pressure amplitude of the pulse. Also, as previously noted, the measured pulse width is becoming wider, whereas the linear theory predicts a more narrow pulse width with increasing hover tip Mach number. In fact, the experimental pulse width (measured at zero pressure) exceeds by at least $50 \%$ the width expected (by linear theory) from an airfoil of chord equal to that of the model rotor tested and traveling at sonic velocity. This pulse-widening effect suggests that aerodynamic events off the rotor blade trailing edge are contributing to the measured acoustic signature.

The difference in peak negative-pressure levels between linear monopole theory and experiment can be seen more clearly in figure 53. The theoretical model does not predict the rate of increase of the peak negative-pressure level. 


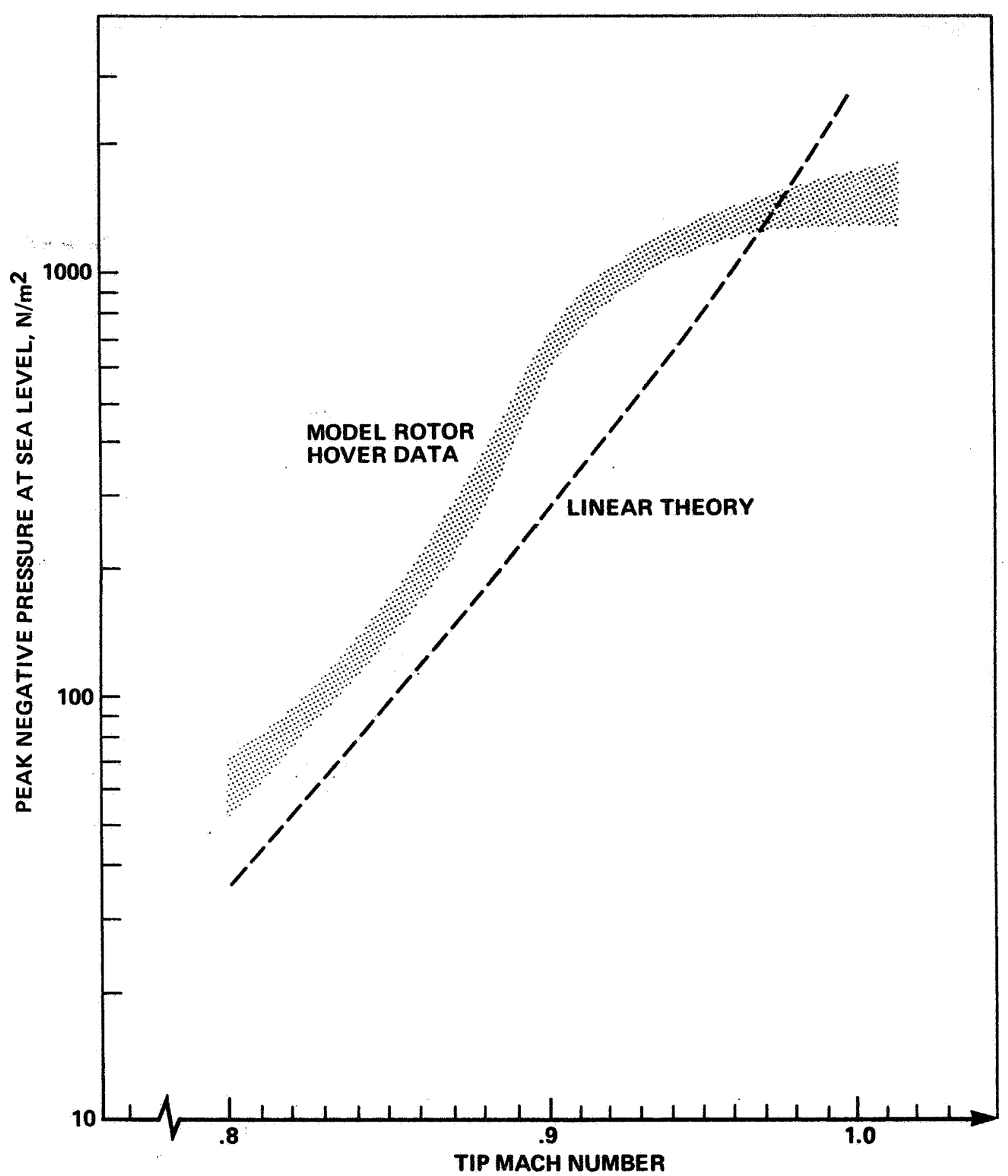

Figure 53.- Comparison of theory and experiment in hover, in-plane, $r_{H} / D=1.5$. 
It is tempting to attribute the lack of correlation with experiment to the simplicity of the linear theoretical model. Perhaps if the remaining linear "dipole" terms were included (the second term in eq. (1)), the agreement with measured data might be better. This was tried in reference 16 for a rotor with predicted values of loading and skin friction suitably chosen to act as pressure dipoles. The resulting theoretical time-histories are almost indistinguishable from the simple linear monopole calculations previously presented. The comparison is shown in figure 54 for hover tip Mach numbers of 0.8 and 0.9 .

These results were not too surprising. Similar conclusions were drawn in reference 8 for a high-speed rotor in forward flight from order-of-magnitude and pulse-shape arguments. In addition, most of the experimental data had suggested that the high-speed noise phenomenon was not dependent on thrust or torque at constant Mach number.

The major conclusion from all of these linear acoustic analyses is that they do not adequately describe the in-plane noise radiation processes from a high-tip-speed hovering rotor. There have been some efforts to correct this situation by improving the modeling (improving the specification of the boundary conditions) in the linear formulation. It can be argued that rotor-tip end-plate (ref. 17) and boundary-layer separation efforts increase the amplitude of the symmetrical pulse. The importance of the latter effect can be seen in figure 55. This end view of a NACA 0012 rotor at near zero lift (ref. 33) is a holographic interferogram of the integrated three-dimensional flow field surrounding the tip of a hovering UH-1H model rotor at the tip Mach number of 0.9 . It is quite apparent that local shock waves on the surface of the blade interact with the boundary layer causing an enlarged separated flow region." A rigorous treatment of this problem is not usually attempted, for it would be necessary to model the boundary layer and separated-flow effects in equation (10). Instead, an "equivalent airfoil" comprising the original airfoil plus the outer edges of the separated-flow region is defined. This new equivalent airfoil is then used in equation (10) to define the strength of the distributed acoustic sources. If this is done, it is relatively easy to show that the peak negative-pressure calculations would increase substantially (they approximately double for each doubling of the effective airfoil chord at constant thickness). A1.though this effect has been known for many years, most rescarchers do not like to incorporate such an estimation in a "first-principles" analysis. The methods of estimating just how thick or extended the separation region is on a three-dimensional rotor in the transonic regions and how to model the equivalent airfoil for noise purposes are not well defined or even completely understood. In addition, none of these corrections predict the development and radiation of the delocalized shock wave above a hover tip Mach number of 0.9 for a scaled UH-1H rotor. Clearly, the radiation processes at these high tip speeds are governed to a large extent by transonic effects. These must be accounted for in the theoretical modeling. 

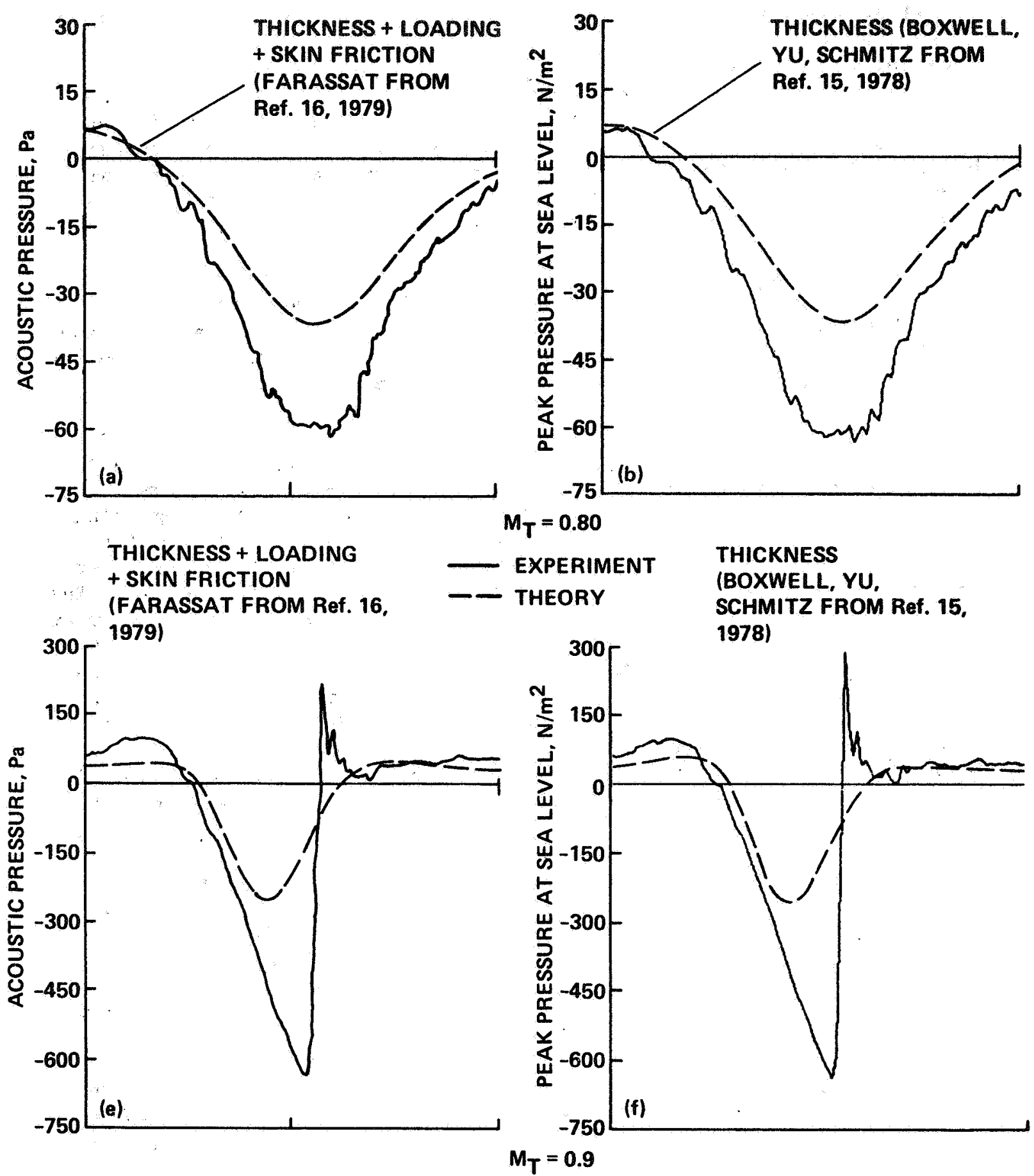

Figure 54.- Comparison of theory and experiment. 


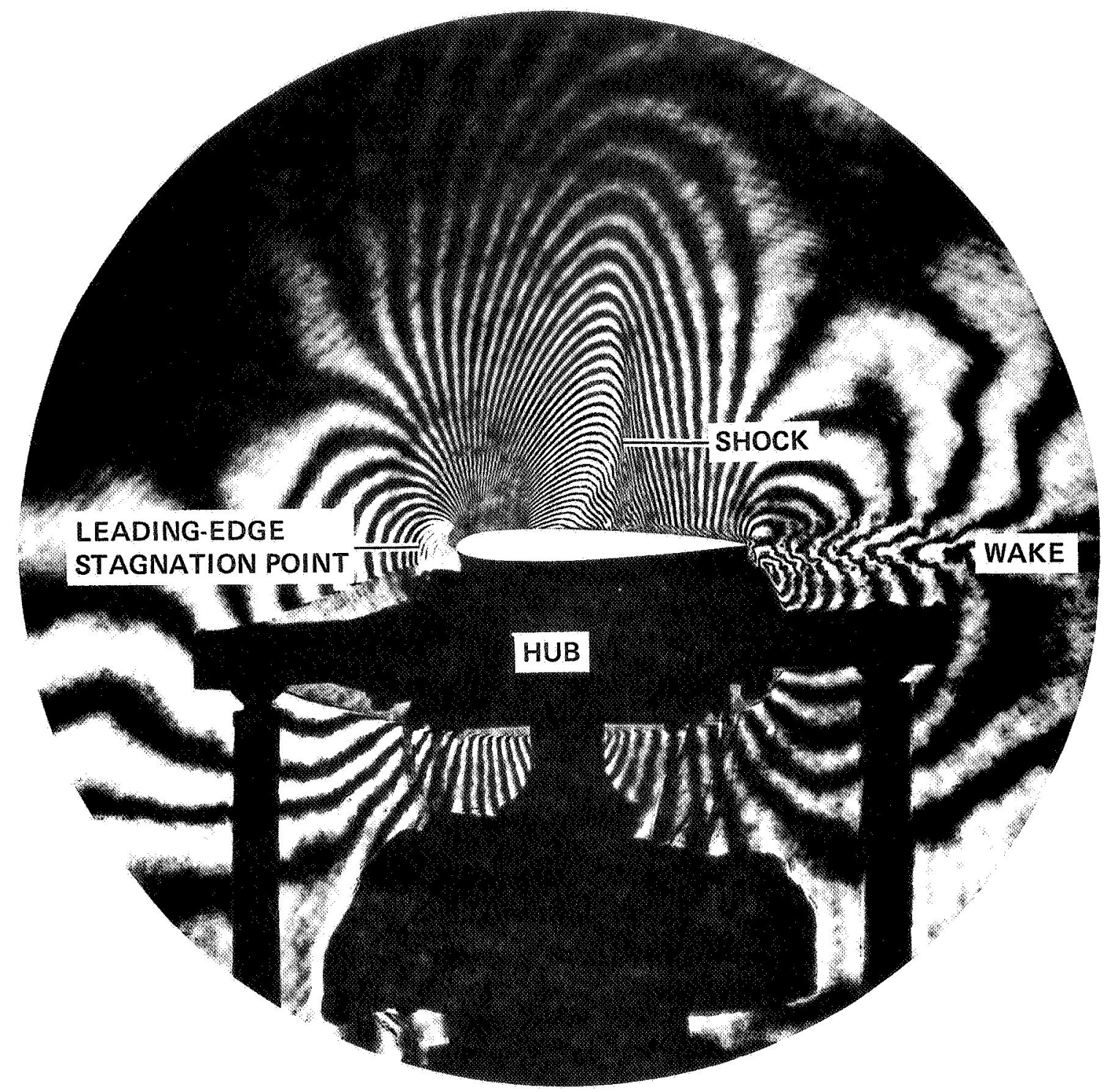

Figure 55.- Interferogram recorded at $180^{\circ}$ (chordwise view).

The most straightforward approach to the nonlinear, high-speed acoustic problem might appear to be to simply include the "quadrupoles" in equation ( 1 ). However, equation ( 1 ) is in reality an integral equation which has no simple analytical solution. Some degree of approximation is necessary to proceed with this approach. These approximations will rely on what we know about the problem physically. Such insight can be gained by switching "hats" and formulating the problem as a transonic aerodynamist. 
We begin with the classical potential equation in a space-fixed coordinate system. Assuming constant specific heats and weak shocks (i.e.s negligible entropy increases), we have

$$
\frac{\partial^{2} \phi}{\partial t^{2}}-a^{2} \nabla^{2} \phi+2 \operatorname{grad} \phi \cdot \operatorname{grad} \frac{\partial \phi}{\partial t}+\frac{1}{2} \operatorname{grad} \phi \cdot \operatorname{grad}\left[(\operatorname{grad} \phi)^{2}\right]=0
$$

where $\phi$ represents the velocity potential and $a$ is the local speed of sound. Fortunately, the aerodynamics of a hovering rotor are basically steady when viewed from a blade-fixed frame. Therefore, following the work of Isom (ref. 19) and references 20-22, the governing potential equation can be transformed to blade-fixed cylindrical coordinates and expanded to second-order yielding the following:

$$
\begin{aligned}
\left\{u^{2}-\frac{a_{0}^{2}}{r^{2}}-(\gamma+1) \frac{\omega}{r^{2}} \phi_{\theta}\right\} \phi_{\theta \theta}-2 \omega \phi_{r} \phi_{r \theta}-2 \omega \phi_{z} \phi_{z} \theta \\
=\left\{a_{0}^{2}+(\gamma-1) \omega \phi_{\theta}\right\}\left(\phi_{r r}+\frac{\phi_{r}}{r}+\phi_{z z}\right)
\end{aligned}
$$

where

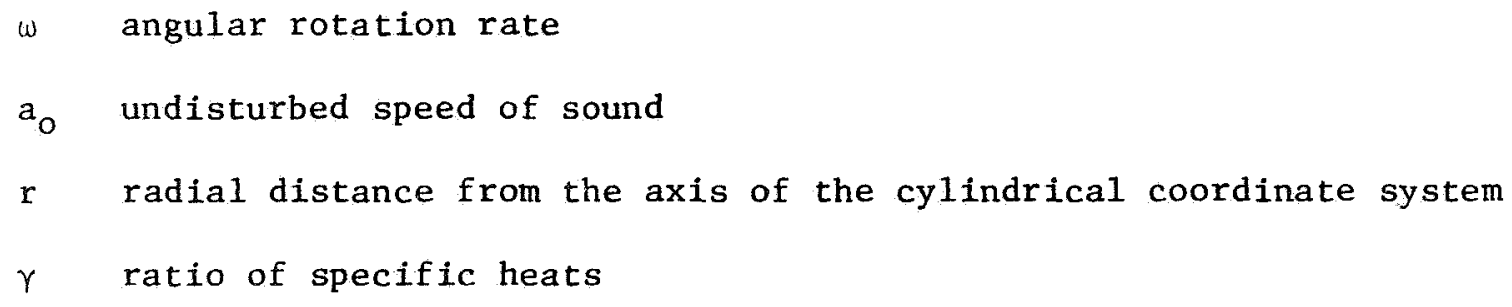

This nonlinear but steady second-order partial differential equation governs the transonic aerodynamics of the hovering rotor. In addition, it governs how disturbances propagate away from this rotating coordinate system (acoustic waves). At the present time, no closed-form solutions to this equation exist. A procedure adopted by some researchers is to numerically solve limited regions of the aerodynamic flow field (refs. 23 and 24). Others have chosen to solve the nonlinear acoustic far-field, using weak-shock theory (ref. 25). As we shall see, neither is a completely satisfactory solution, for the nonlinear aerodynamic and acoustic fields are interwoven.

The choice of a cylindrical coordinate system whose axis is aligned with the rotor is sketched in figure 56 . An observer riding in this coordinate system sees a free-stream velocity that increases 1inearly from zero at the origin to $\omega r$ at $r$. As indicated, this increasing free-stream velocity continues out past the tip of the rotor; it will be shown to be important to many of the arguments to come. 


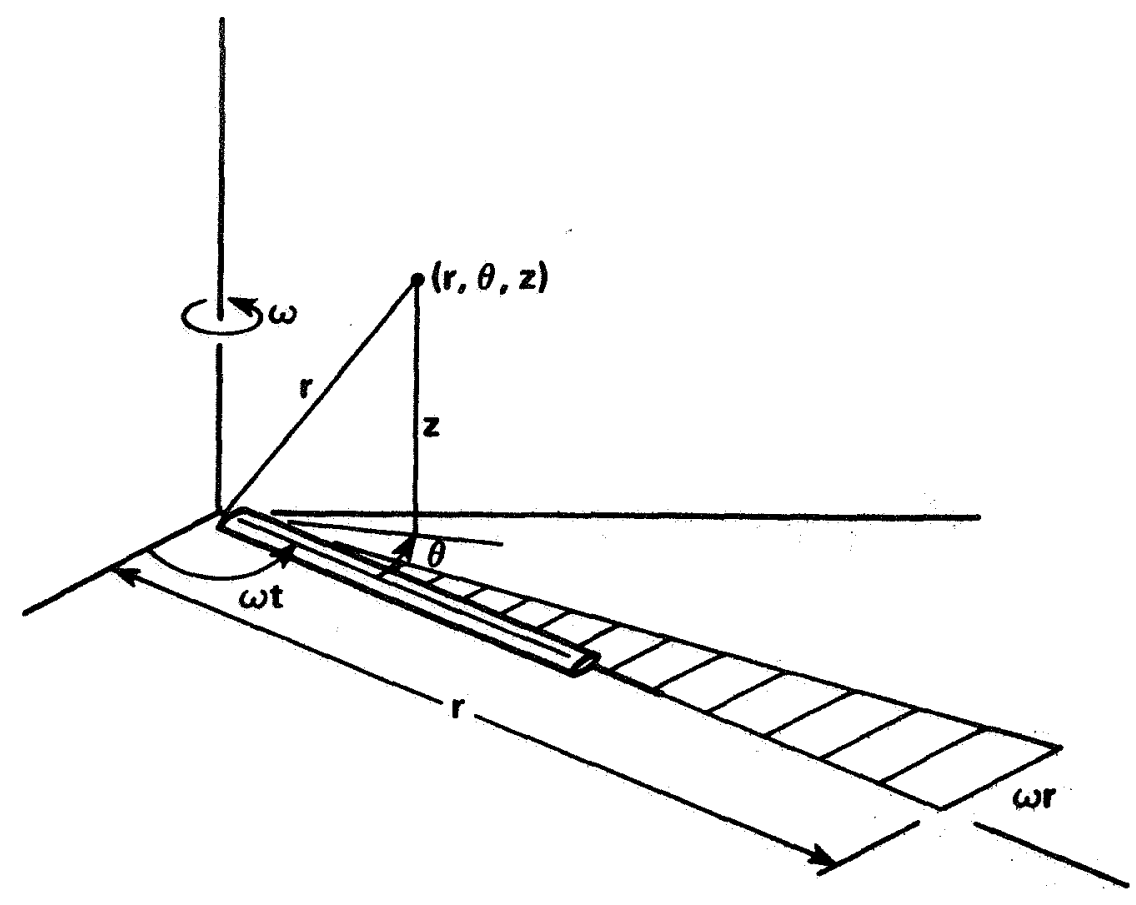

- Figure 56.- Cylindrical coordinate system.

Before attempting to solve equation (17), it is instructive to follow the approach of references 19 and 21 and explore the behavior of the governing equation. It is known from the theory of partial differential equations that the coefficient of $\phi_{\theta \theta}$ governs the general character of the potential equation. For when

$$
\begin{aligned}
A \equiv & \omega^{2}-\frac{a_{0}^{2}}{r^{2}}-(\gamma+1) \frac{\omega}{r^{2}} \phi_{\theta} \\
& <0: \text { elliptic behavior } \\
& >0: \text { hyperbolic behavior }
\end{aligned}
$$

However, A takes a more recognizable form after some further manipulation:

$$
A=\omega^{2}-\frac{a_{0}^{2}}{r^{2}}-\frac{(\gamma-1) \omega}{r^{2}} \phi_{\theta}-\frac{2 \omega}{r^{2}} \phi_{\theta}
$$

Using the energy equation, this becomes

$$
A=\omega^{2}-\frac{a^{2}}{r^{2}}-\frac{2 \omega \phi_{\theta}}{r^{2}}
$$


Defining $U_{\infty}=\omega r, \phi_{\theta}=-u r$, we obtain

$$
A=-\frac{a^{2}}{r^{2}}\left\{1-\frac{\left(U_{\infty}+u\right)^{2}}{a^{2}}+\frac{u^{2}}{a^{2}}\right\}
$$

The last term in the brackets of equation (18) can be neglected. It is second order to $A$ and a third-order correction to the second-order potential equation. If we now define

$$
\mathrm{M}_{\ell} \equiv \frac{\mathrm{U}_{\infty}+\mathrm{u}}{\mathrm{a}}=1 \text { ocal Mach number }
$$

the coefficient of $\phi_{\theta \theta}($ eq. (17)) becomes

$$
A=-\frac{a^{2}}{r^{2}}\left(1-M_{\ell}^{2}\right)
$$

Therefore, the general behavior of the second-order transonic potential equation is governed by the local Mach number of the flow. If $M_{\ell}<1.0$, then $A<0$, and the governing equation is elliptic. In this case, no wave-like structure is possible. However, if $M_{\ell}>1.0$, then $A>0$, and the governing nonlinear partial differential equation is hyperbolic. Then, characteristics are formed along which disturbances can propagate in a wave-like manner. It is also important to realize that the local Mach number $M_{\ell}$ is dependent on the free-stream velocity $\omega r$, the local speed of sound $a$, and the local perturbation velocity $u=-\phi_{\theta} / r$. Each contribution. is separately identifiable in equation (19).

These ideas are quite useful when one is attempting to explain the phenomenon of delocalization for the hovering rotor experiment described previousiy. This connection was theoretically suggested in references 22 and 25 , numerically calculated in references 20 and 21 , and experimenta11y confirmed in reference 22 . In the following paragraphs, the relationships are experimentally shown to depend on the local Mach number of flow. Three distinct cases are considered: $M_{T}=0.85,0.88$, and 0.90 . Some freedom has been taken with the graphics in the interest of presenting a clear picture of the basic relationships involved. The data are the same as those reported in reference 20. In the figures that follow, the top views are sketches of events pieced together with limited experimental data, and the aft views are, for the most part, interpolations of experimental data.

Figure 57 depicts the top and aft views of a rotor operating at a free-stream tip Mach number $\left(M_{T}\right)$ of 0.85 . A locally supersonic region exists near the tip of the rotor. For this region $M_{\ell}=(\omega r+u) / a>1.0$, even though $w r / a_{o}$ all along the blade span is less than 0.85 . The hyperbolic nature of this pocket of supersonic flow is a result of local aerodynamic nonlinearities (i.e., changes in the local speed of sound, $a$, and local perturbation velocity, $u$ ). Surrounding this locally supersonic 


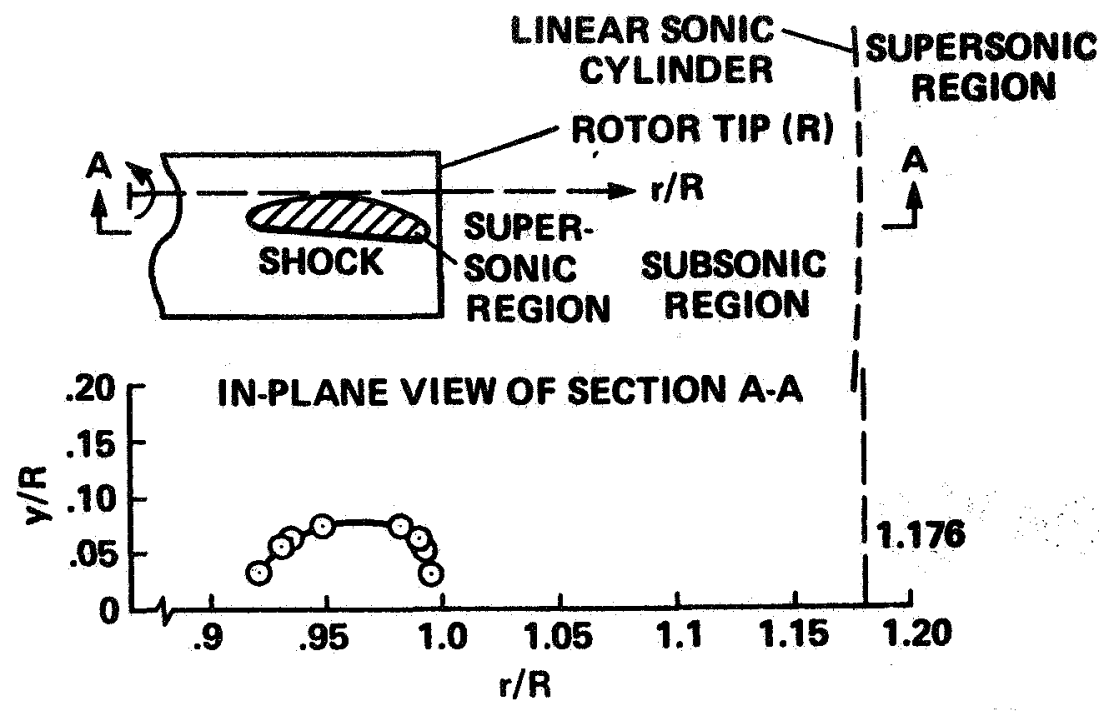

Figure 57.- Top and aft views of shock boundaries of a rotor, $\mathrm{M}_{\mathrm{T}}=0.85$.

flow is a subsonic flow region, $M_{\ell}<1.0$, in which the governing potential equation is elliptic. No wave-like behavior is possible through this compressible elliptic region. However, as $r$ increases beyond the $t i p$ of the blade, $M_{\ell}$ again becomes greater than 1 because of the linearly. increasing free-stream velocity field of the blade-fixed cylindrical coordinate system. For this region $u \neq 0$, $a=a_{0}$, so

$$
M_{\ell} \approx \frac{\omega r}{a_{0}}>1.0
$$

The surface where this first happens has been called the sonic cylinder (refs. 22 and 25). At radii larger than the sonic cylinder, the equation again becomes hyperbolic, and wave-like propagation is certain. The acoustic implications of this 0.85 case begin in the hyperbolic pocket of flow near the blade tip. Wave-like disturbances in this region terminate on the boundary of an elliptic region where they no longer propagate in characteristic directions. The wave-like character of the inner pocket is thus broadened as information passes through the elliptic region to the sonic cylinder. These broadened disturbances are then propagated in a wave-like manner throughout the outer hyperbolic region. The result is a smoothly varying, near-symmetrical acoustic signature in the far-field.

The competing phenomena become even more interesting when the undisturbed free-stream tip Mach number of the rotor $\left(M_{T}\right)$ is increased to 0.88 ( $\mathrm{fig}$. 58). The inner supersonic (hyperbolic) region grows and extends off the tip of the rotor - again being driven by local aerodynamic nonlinearities. At the same time, the higher free-stream tip Mach 


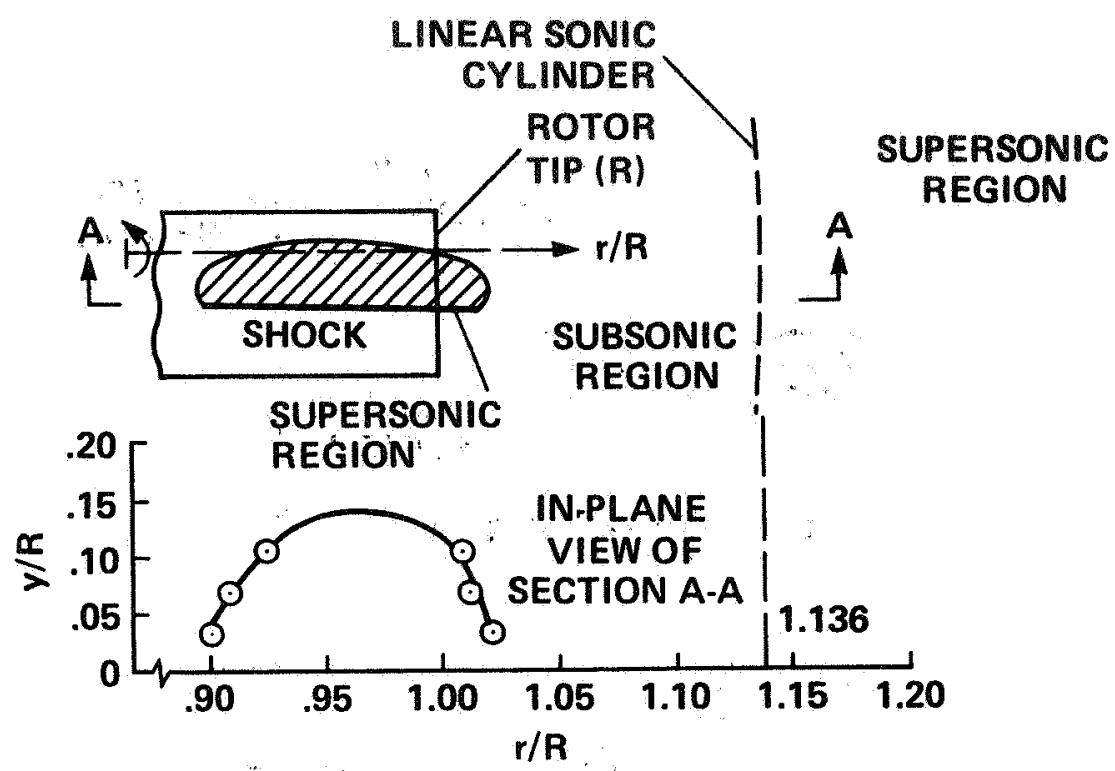

Figure 58.- Top and aft views of shock. boundaries of a rotor, $\mathrm{M}_{\mathrm{T}}=0.88$.

number of the rotor decreases the radius of the sonic cylinder, thus moving the outer hyperbolic region toward the rotor tip. In addition, the proximity of the linear sonic cylinder to the blade tip introduces aerodynamic nonlinearities. These tend to warp the sonic cylinder inward, bringing the two hyperbolic regions even closer together. However, the hyperbolic regions do not overlap, thus ensuring that 1ocally generated waves in the inner region do not propagate along characteristics to the hyperbolic far-field. Instead, the wave-like disturbances are forced to pass through a small elliptic region where they are broadened before entering the outer hyperbolic region for propagation to the far-field. The resulting acoustic signature becomes more sawtoothed in character, but does not contain radiating shocks.

The last and most interesting condition, in which the local freestream tip Mach number is increased to 0.9 , is sketched in figure 59 . The localized inner hyperbolic and outer hyperbolic regions connect off the blade tip, forming one continuous supersonic region $\left(M_{l}>1.0\right)$. In this case, shock waves that are generated on the surface of the rotor now propagate uninterruptedly in a radially outward characteristic direction to the acoustic far-field. The resulting delocalization phenomenon is quite striking, for the character and the intensity of the acoustic signature change dramatically. At all three of these conditions, measured values of local Mach number support and explain the phenomenon of "transonic delocalization." 


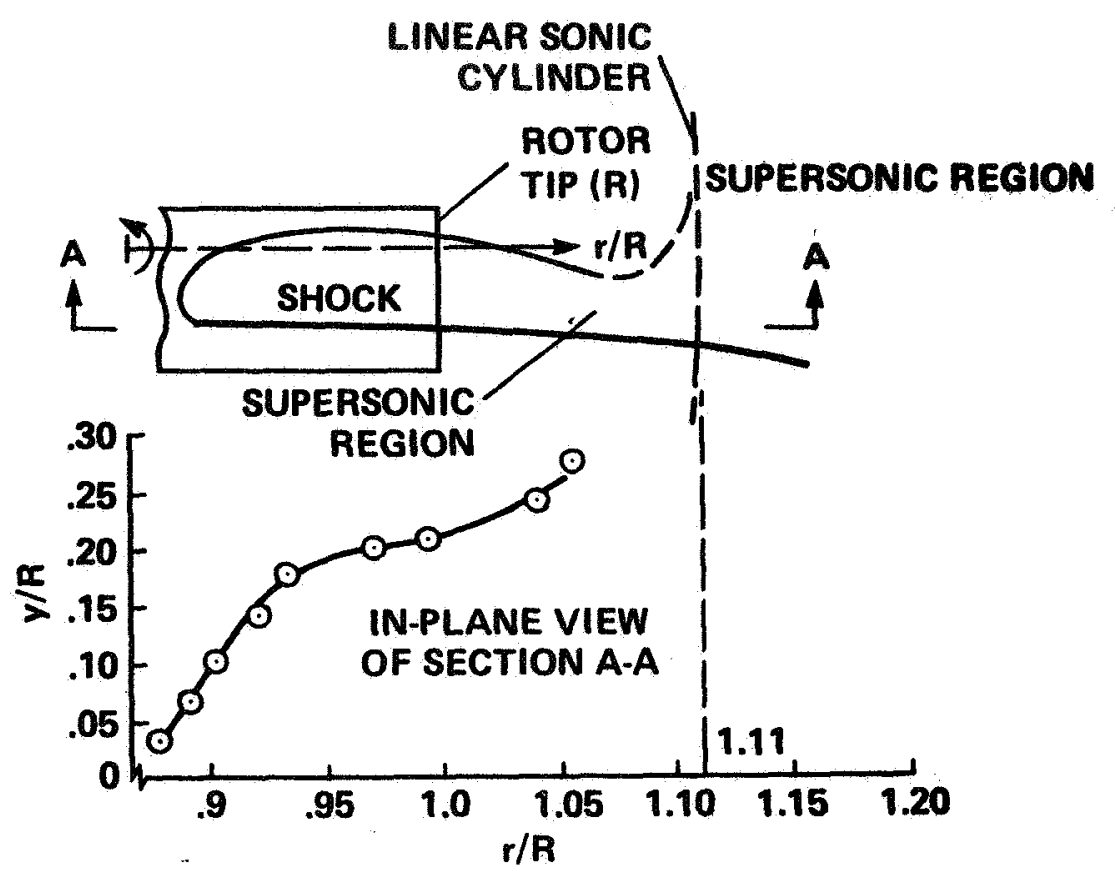

Figure 59.- Top and aft views of shock boundaries of a rotor, the development of delocalization phenomenon, $\mathrm{M}_{\mathrm{T}}=\mathbf{0 . 9}$.

Further confirmations of the mechanism of delocalization can be made theoretica1ly using existing transonic aerodynamic codes (see refs. 26-28). An example calculation for this rotor in hover is shown in figure 60 , the work of Shenoy (ref. 29) using the transonic code of reference 27 . The agreement between theory and experiment is quite good - conciusively demonstrating the interrelationship between transonic and high-speed rotor noise.

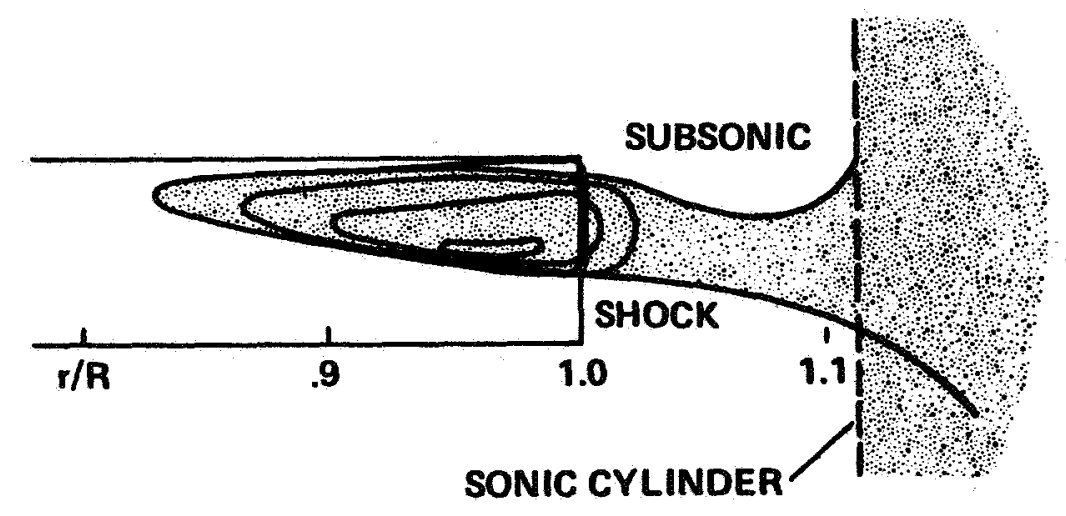

Figure 60.- Tip flow field of a hovering rotor $\left(M_{T}=0.9\right)$. 
Although the phenomenon of delocalization has been explained by simply looking at the coefficient of $\phi_{\theta \theta}$ in equation (17), predicting the radiating acoustic field is another matter. The explanations presented are themselves functions of either measured or calculated flow properties. In essence, a near-field description of the aerodynamic flow field is required before the events in the acoustic far-field can be explained. Even then, acoustic nonlinearities in equation (16) may alter the waveform of the propagating wave (ref. 25). Precise calculations of the radiating sound field are dependent on the full solution of the nonlinear potential equation (ref. 25).

On the other hand, the successful explanation of the delocalization phenomenon suggests that local aerodynamic nonlinearities strongly influence the acoustic radiation problem. Therefore, a logical step in the calculation of the acoustic field is the incorporation of the nearfield aerodynamic nonlinearities in the acoustic radiation equation.

At least two alternative ways of implementing this idea have been presented in the literature. In one proposed method (ref. 21) the nonlinear near-field is mapped to a nonrotating control surface, where Kirchoff's theorem is applied. As reported, the control surface must be chosen to be large enough to capture the nonlinear aerodynamic behavior of the problem, but not to be so large as to make numerical computation impractical. Calculations using this procedure, coupled with an existing near-field numerical code, have shown improvement in peak amplitude levels; but not much improvement in waveform characteristics above the delocalization Mach number. As discussed in the paper, this is most likely a result of the numerical insensitivity of the transonic code at the boundary of the nonrotating control surface.

In the second method, the we11-known "acoustic analogy" procedures are used to evaluate the volume distributions of local aerodynamic nonlinearities or quadrupoles (refs. 8, 20,22, and 30). The analysis begins with equation (1) (Ffowcs Williams and Hawking). The third term in this equation is the linear thickness contribution to the radiated noise, which by itself has shown the poor agreement with experiment, as previously discussed. The second term, also a surface integration over the blade, can be classified as either a linear or a nonlinear term, depending on how the surface pressure is approximated. However, for most of the in-plane computations reported here, its contribution to the radiated noise field is small and thus has been neglected. A strict evaluation of the nonlinear quadrupole (first) term in equation (1) requires a volume integration over all space. However, the interest in this paper is in capturing those nonlinear aerodynamic terms that may govern the acoustic radiation problem. Therefore, the evaluation of the quadrupole term is confined to a volume integration within the aerodynamic nonlinear flow field of the blade. 
The quadrupole term assumes a simpler form if we restrict our attention to the acoustic far-field. Then the spatial differentiations can be easily converted to time differentiations. The first term of equation (1) becomes

$$
\frac{\partial^{2}}{\partial x_{i} \partial x_{j}} \int_{v}\left[\frac{T_{1 j}}{r\left|1-M_{r}\right|}\right] d V \cong \frac{1}{a_{0}^{2}} \frac{\partial^{2}}{\partial t^{2}} \int_{v}\left[\frac{T_{r r}}{r\left|1-M_{r}\right|}\right] d V
$$

where $T_{r r}=T_{i j} \hat{r}_{i} \cdot \hat{r}_{j}$ and $\hat{\mathbf{r}}$ is the unit vector from the source at the retarded time to an observer in the acoustic far-field. It is known from transonic computations and experimentation that the primary quadrupole regions are confined within a few chord lengths normal to the rotor plane.

For in-plane far-field radiation, the unit vector $\hat{r}$ is nearly in the blade rotational plane and is nearly parallel to the blade chordwise direction when the acoustic pressure reaches its peak level. If isentropic flow is assumed and the perturbation velocities are measured in the coordinate system given in figure $56, \mathrm{~T}_{\mathrm{rr}}$ becomes

$$
T_{r r}=\rho_{0}\left(v_{\theta}^{2} \cos ^{2} \theta+2 v_{r} v_{\theta} \cos \theta \sin \theta+v_{r}^{2} \sin ^{2} \theta\right)+\frac{r-1}{2} \rho_{0}\left(\frac{\omega r}{a_{0}}\right)^{2} v_{\theta}^{2}
$$

where the $z$-component of the perturbation velocity does not appear because of the choice of an in-plane far-field microphone position. For simplicity in the resulting calculations, it has been assumed that $\sin \theta \approx 0$ and that $u \approx v_{\theta}$ near the integration region of interest. This is true as long as the quadrupole field is in fact localized to a region near the rotor tip. Then,

$$
T_{r r} \cong \rho_{0} u^{2} \cos ^{2} \theta+\frac{\gamma-1}{2} \rho_{o}\left(\frac{\omega r}{a_{0}}\right)^{2} u^{2}
$$

where $u$ represents the perturbation velocity along the blade chord and wr is the free-stream velocity of the point in the flow field being evaluated. The two terms represented in equation (23) arise from similar properties of the flow already discussed in the potential formulation. Changes in the local speed of sound and local streamwise perturbation nonlinearities are included, although the equation forms do not permit a one-to-one correspondence of terms.

Equations (1), (21), and (23) described the nonlinear far-field acoustic radiation of the transonic hovering rotor. For subsonic tip Mach numbers, numerical evaluation of the surface integrals presents no real problems. However, the volume integration of quadrupoles is not as straightforward. 
As discussed previously, the accuracy of this nonlinear formulation is totally dependent on the detailed knowledge of the flow field surrounding the blade. In this paper, the flow fields of interest were computed by the three-dimensional transonic numerical code described in references 23 and 24 . This code solves the near-field transonic sma11disturbance potential equation in a blade-fixed reference frame. The solution uses a conservative, mixed-difference relaxation scheme. Because this code was developed to predict blade-surface aerodynamics, some simplifying assumptions were made to equation (17); namely, the nonlinearities known to be small near the blade surface were neglected. In particular, the terms $2 \omega \phi_{\mathrm{r}} \phi_{\mathrm{r}} \theta$ and $2 \omega \phi_{z} \phi_{z} \theta$ on the left-hand side and the factor $(\gamma-1) \omega \phi_{\theta}$ on the right-hand side of equation (17) were not programmed.

The nature of the calculated chordwise pressure distributions as a function of blade radius is shown in figure 61 for a tip Mach number of 0.9 at zero angle of attack. On the blade surface, a shock wave forms at about $90 \%$ of the span and persists over the outer portions of the blade and beyond. These calculated pressure distributions are consistent with the experimentally sketched flow regions of figure 59. The maximum value of shock strength is calculated to occur at $95 \%$ radius. It is also important to note that the shocks that are calculated with this code do not look like measured shock waves. The numerical solution has built into it a numerical viscosity which tends to smooth discontinuities over several mesh points. This affects the final acoustic waveforms to some degree and is discussed later.

The final problem in the evaluation of the quadrupole integral is the development of a calculation procedure for equations (1), (21), and (23), that is valid when $M=1.0$. This problem occurs when the volume integration is extended up to and beyond the linearized sonic cylinder.. The integrand in equation (21) then contains the product of two terms which compete to decide the eventual magnitude of the quadrupole radiation. The first is the decaying source field represented by the $\mathrm{T}_{\mathrm{rr}}$ term in equation (21). This is multiplied by the $1 /\left(1-\mathrm{M}_{\mathrm{r}}\right)$ term which goes to $\infty$ as $M_{r}$ approaches 1 . Fortunately, the singularity is integrable, but it must be handled quite carefully. In the results summarized here, the acoustic planform technique was chosen to perform the numerical integration near $M_{r}=1.0$. A complete discussion of the procedures and pertinent references is given in reference 20.

An evaluation of the prediction accuracy is presented below by comparing theory with the same UH-1H hover rotor data. Figure 62 presents the monopole and quadrupole contributions to the radiated noise at $M_{\text {tip }}=0.88$ (slightly before delocalization). At this Mach number, the shape of the quadrupole term is basically still symmetrical - however, some asymmetrical character is present on the pressure recovery side of the quadrupole calculation. When the monopole and quadrupole contributions are added, good correlation in amplitude and pulse shape is observed (fig. 62). The overall shape of theory and experiment are still basically 
- $c_{p}$ ON THE BLADE SURFACE

- - $c_{p}$ AT 1.18 CHORD UP VERTICALLY

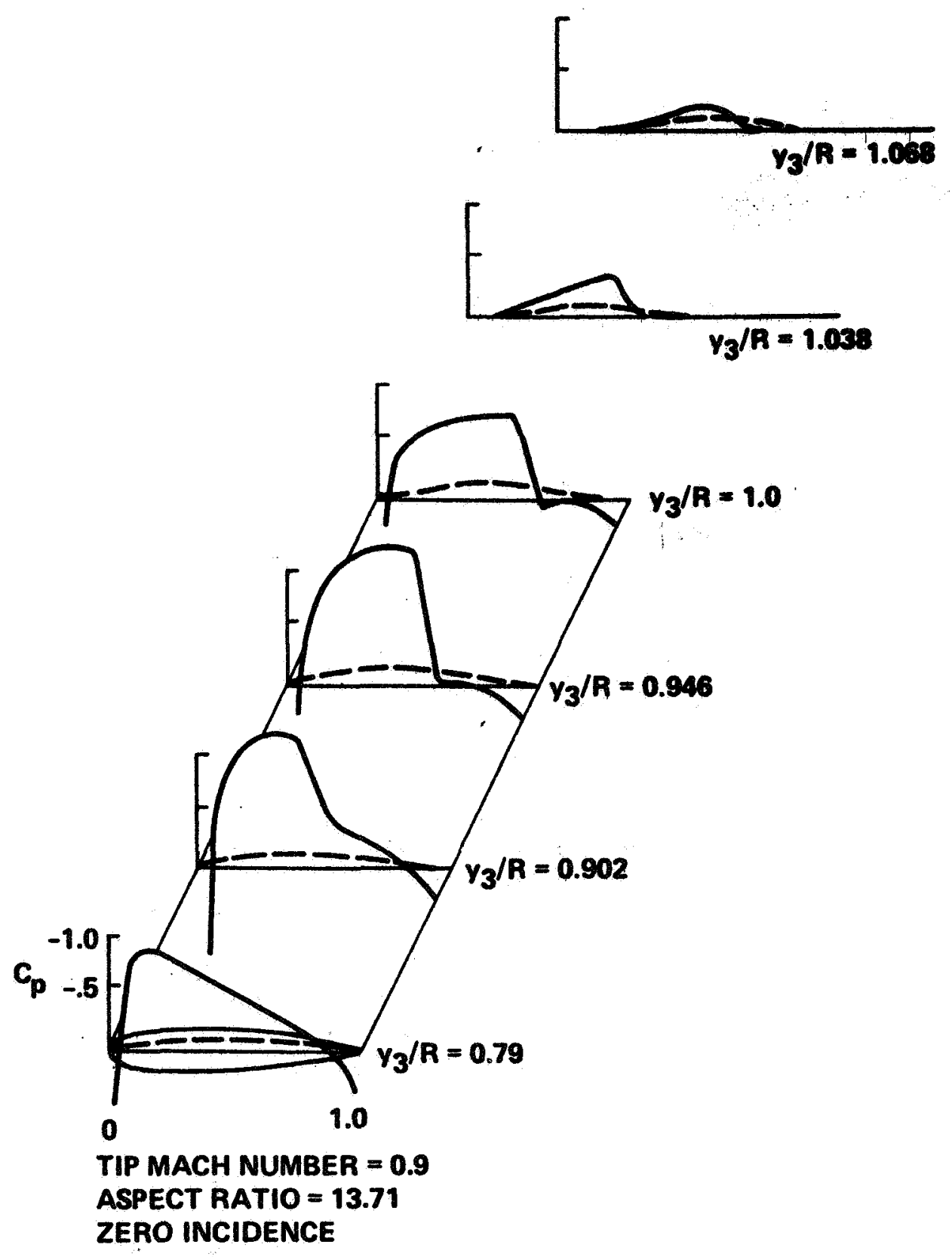

Figure 61.- $c_{p}$ distribution on the blade surface and at 1.18 chords up. aspect ratio $=13.7$, NACA 0012 , near zero angle of attack, $M_{T}=0.9$. 


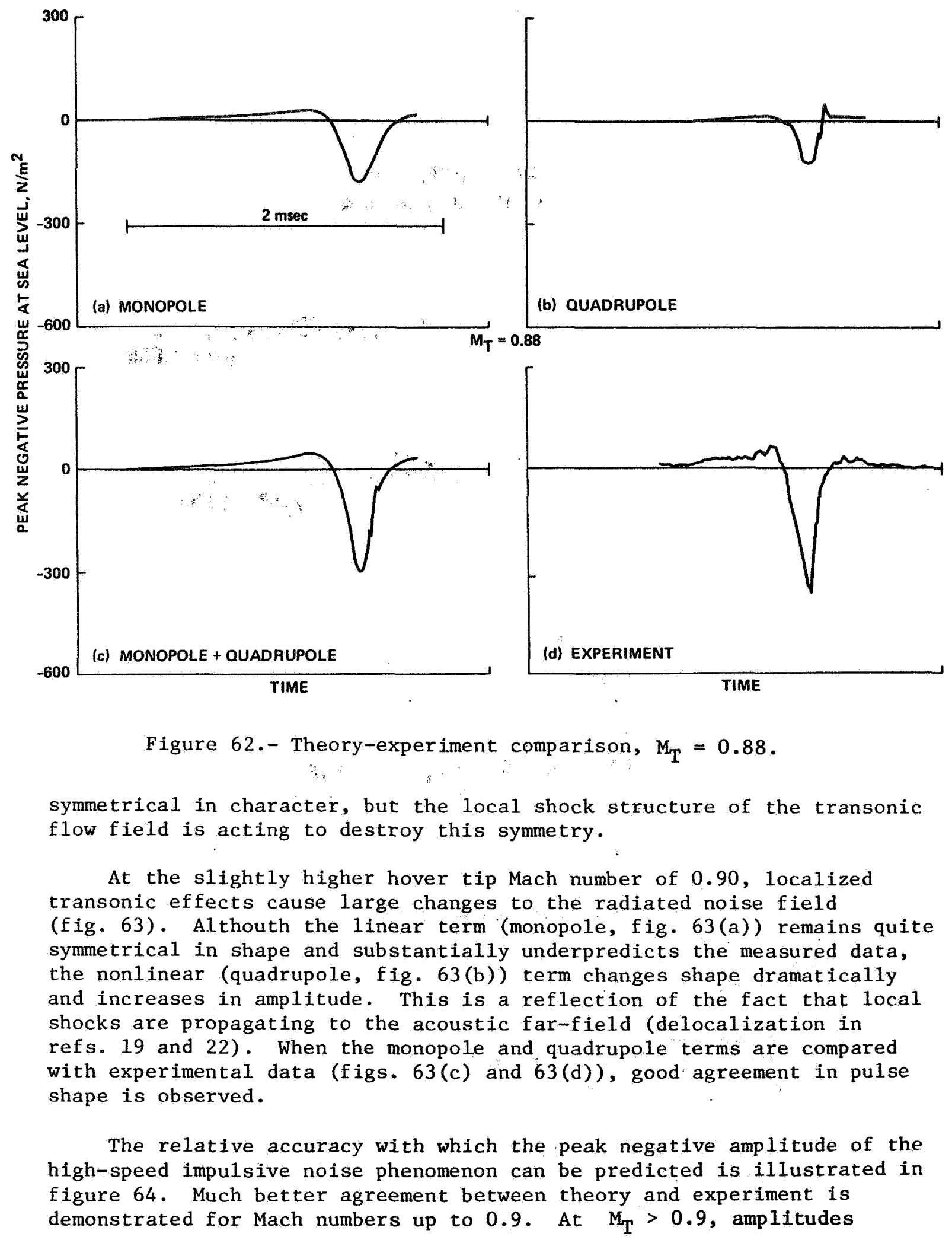




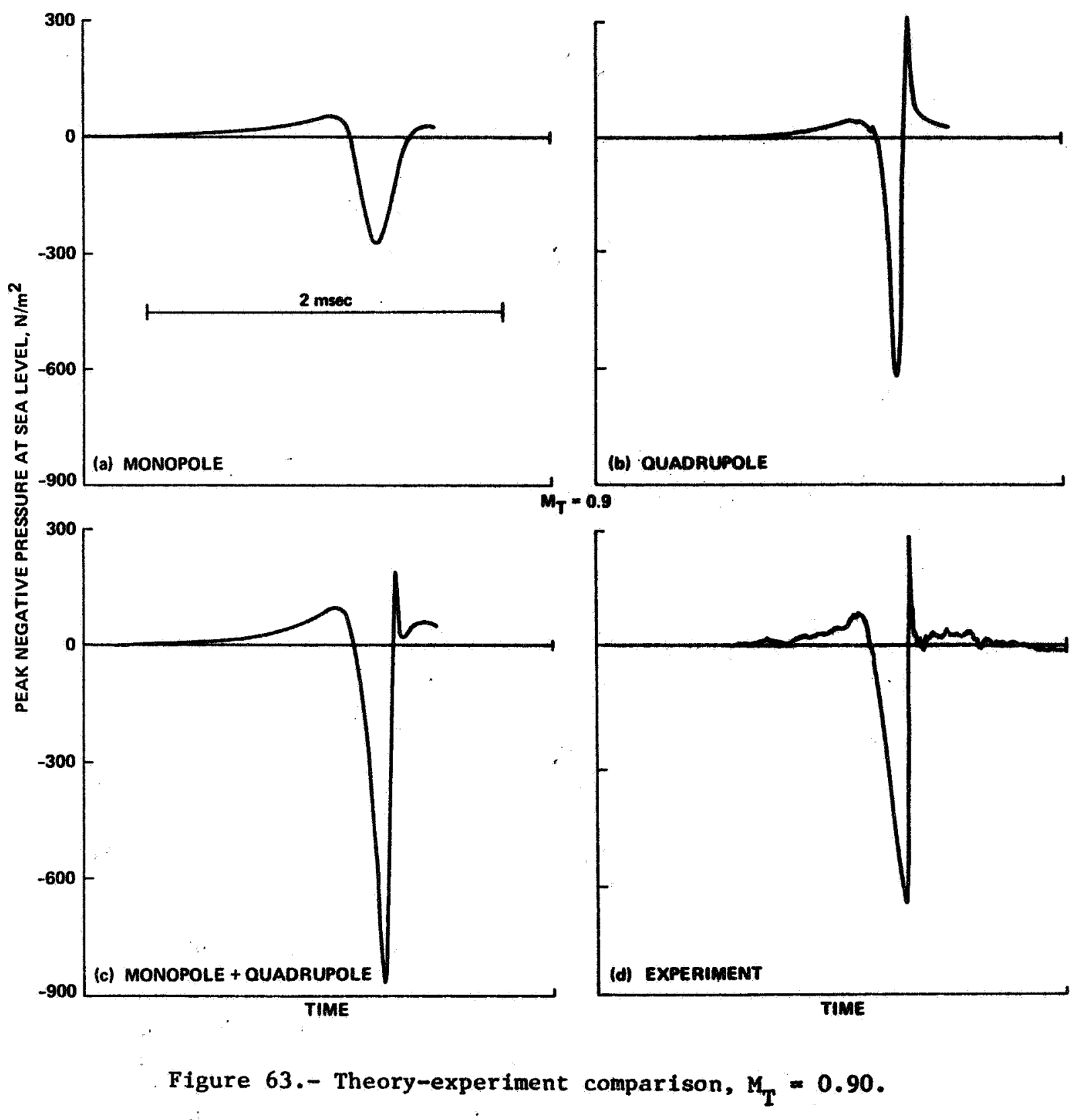




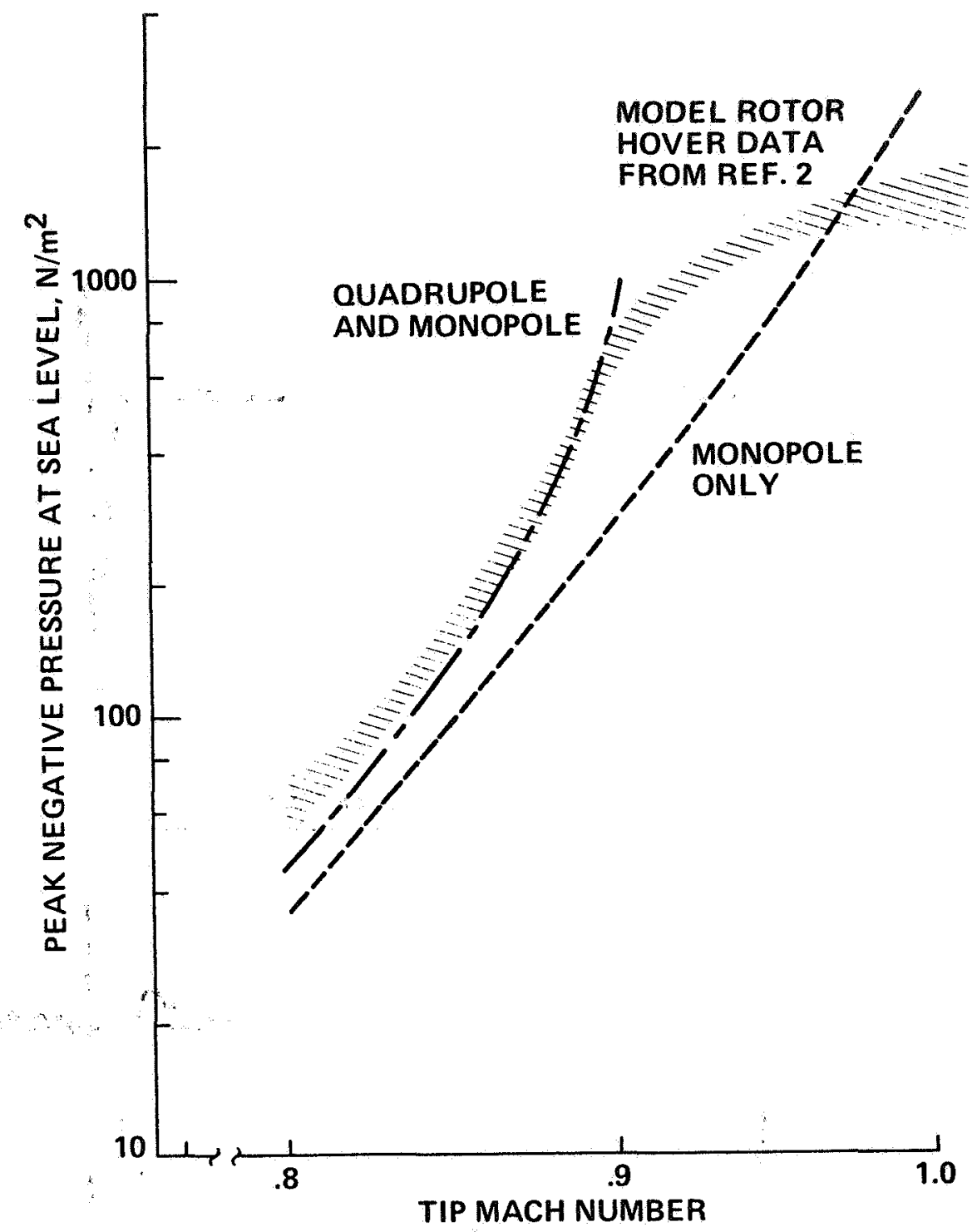

Figure 64.- Comparison of theory and experiment in hover, in-plane, $\mathbf{r}_{\mathrm{H}} / \mathrm{D}=1.5$.

are overpredicted. Additional time-history comparisons can be found in reference 20 .

The most exciting part of the high-speed impulsive noise problem is that we have come so far in understanding and predicting the basic physical phenomena. Nevertheless, the computation of the noise is not routine. It remains a difficult task involving a matching between a near-field transonic code and a suitable acoustic code. Several areas where improvements can and are being made (in some cases) are suggested in the following paragraphs. 
The high-speed impulsive noise prediction process is dependent on the quality of the transonic near-field aerodynamic data. At the present time, existing numerical codes contain "numerical viscosity" which tends to smear the local shock structure. This smearing is most pronounced off the blade surface - past the blade tip - and can cause numerical errors in either the Kirkoff (ref. 21) or quadrupole acoustic formulations. Some alternative transonic formulations that use shockcapturing methods may help better define these important regions of the flow field.

It is also possible to study numerical and physical aspects of the delocalization process by considering a related two-dimensional problem wave propagation from disturbances emanating from a physical rotating cylinder (ref. 31). In essence, a cylinder is mounted between two end plates so that its axis of rotation is at right angles to the plates. A "bump" is fastened to the cylinder surface aligned so that the cross section of the bump and the cylinder are the same along any point on the axis of the cylinder (see fig. 65). The cylinder and bump are rotated at transonic circumferential Mach numbers simulating the delocalization flow field

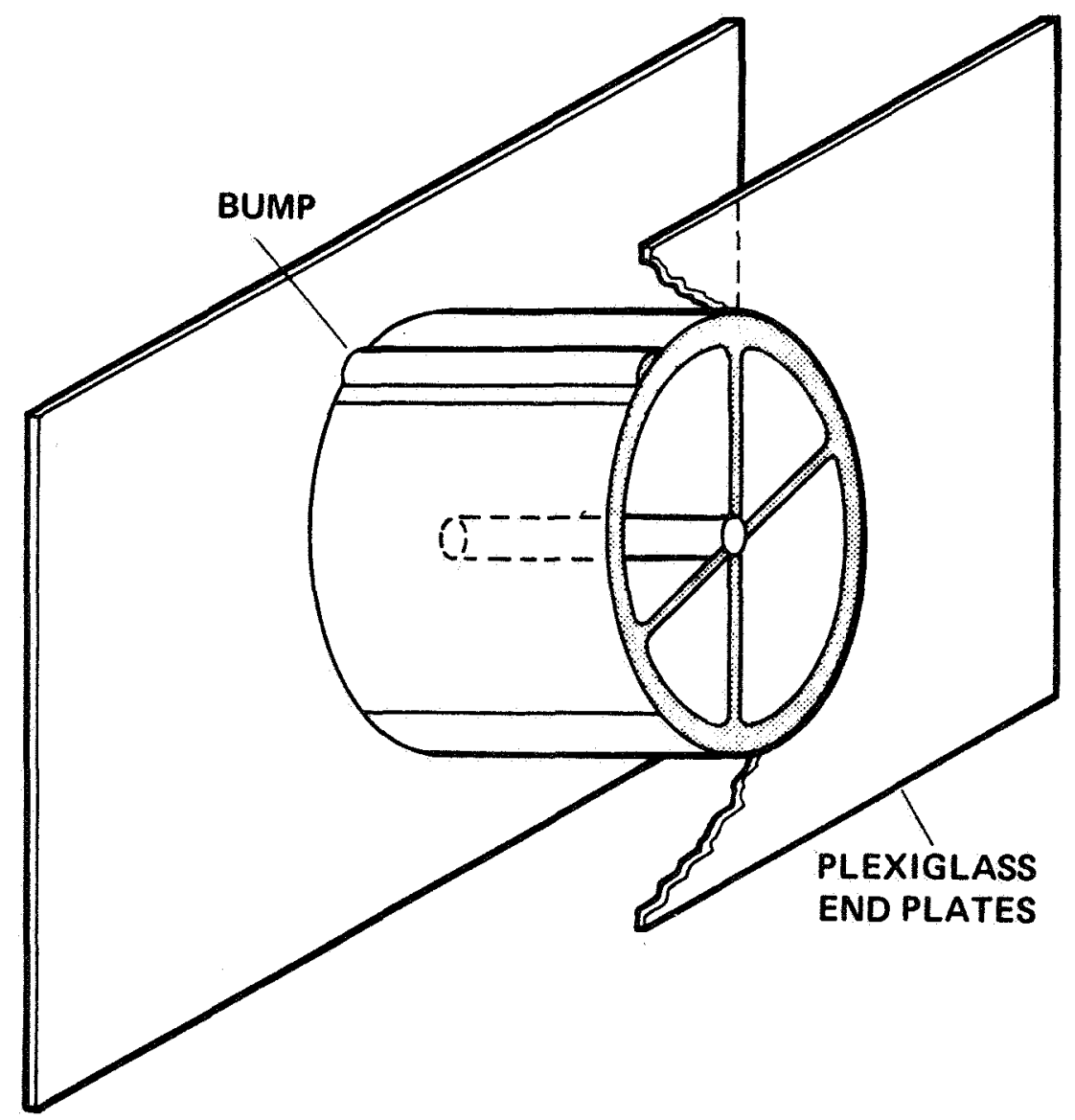

Figure 65.- Schematic of a rotating cylinder. 
of the hovering rotor. The end plates and geometrical designs make this simulated problem two-dimensional, thus making it much easier to investigate the numerical sensitivity of the transonic delocalization process. Initial computations are quite promising (ref. 31). In the near future, complementary experimental testing will commence.

In addition to numerical considerations, new experimental methods are needed to develop quantitative methods to measure the rotor tip flow field. of course, blades instrumented with pressure transducers near the rotor tip yield invaluable data on the surface of the blade (ref. 32). However, the flow field off the rotor blade tip is perhaps even more important for transonic rotors. In the past, hot-wire anemometry has been successfully used to measure the transonic flow field (ref. 22). More recently, these measurements were charted using laser velocimetry. In each case the velocity field surrounding the rotor-blade tip extending past the blade's sonic cylinder has been extensively mapped and used as input to equation (1), to predict the acoustic far-field. The major problem with either the hot-wire or the laser velocimeter approaches is that they are time consuming.

A quite promising new method for obtaining the flow-field measurements employs holographic interferometry (refs. 18 and 33). A laser beam is expanded and passed through the rotor blade very quickly, mixed with a reference wave, and stored as a hologram of the three-dimensional flow field. A second beam is passed through the same path with the rotor at rest and a second hologram is made. These two holograms are then superimposed, creating an interferogram of the integrated three-dimensional flow field. Figures from reference 18 present a holographic interferogram of a hovering transonic UH-1H model rotor (see fig. 55). By simply counting fringes in the interferogram it is possible to quantitatively define the integrated density of the flow field. However, if many photographs similar to this are gathered at many different angles, it is possible to use the known techniques of tomography to define quantitatively the density of any point in the flow field. This truly exciting prospect has been simulated by successful research at our laboratory, at Stanford University, and at the University of Michigan. The next few years should see the development of holographic interferometric tomography for compressible rotor problems.

Finally, the acoustic calculation process, or coding, still leaves room for improvement. In quite a few cases of interest, including the evaluations of the quadrupole near the linear sonic cylinder region, it is necessary to evaluate an integral while integrand is singular but integrable. This is normally done by using "acoustic planform methods" (refs. 20, 34, and 35). However, it is the authors' opinion that the codes that calculate the far-field acoustics near singularities could be simplified and improved by using more of the physics of the transonic rotor. These improvements are being investigated by researchers at the Aeromechanics Laboratory. 


\section{BLADE-VORTEX INTERACTION IMPULSIVE NOISE}

The blade-vortex interaction impulsive noise story is not as complete as that of high-speed impulsive noise - although neither is totally understood. As discussed at the beginning of this article, the problem is quite complex because it depends strongly on the local aerodynamic state of the helicopter. There have been many excellent experimental and theoretical approaches to this difficult problem over the past 15 years. Although each effort showed progress in understanding, only a few rather qualitative design changes have resulted.

It is the authors' opinion that more definite progress has not been made because we are not sure to what degree we are in fact modeling (theoretically or experimentally) the full-scale aerodynamic sources of interest. For example, it is becoming common practice to gather impulsive-noise acoustic data on a model rotor in an acoustically treated wind tunnel. However, it is of ten not known quantitatively how these data relate to measured noise on the full-scale vehicle. It is not too surprising then if the design changes based on these model results do not prove effective on the full-scale helicopter.

The review of blade-vortex interaction (BVI) impulsive noise presented below is based on the following three fundamental questions:

1. Can BVI impulsive noise be scaled, and what are the known scaling parameters?

2. What are the known physical origins of BVI impulsive noise?

3. How accurately can BVI impulsive noise be predicted?

All of the data and arguments to be presented are for the two-bladed, single-main-rotor helicopter class. Because it has been, historically, the most notable contributor to BVI impulsive noise, much of the BVI research has concentrated on this class of helicopters. However, it should not be concluded that three- and four-bladed helicopters do not generate impulsive noise. On the contrary, as shown in figure 28, fourbladed helicopters can generate impulsive noise as well. In many instances, these noise sources are the dominant source of radiated acoustic energy. (It should be noted that tandem-rotor helicopters also generate BVI impulsive noise by some of the same source mechanisms as the single-rotor helicopter (refs. 36 and 37).)

Another reason for focusing on the two-bladed BVI impulsive noise problem is the relative simplicity of the two-bladed teetering system. The rotors themselves are of ten large with minimal dynamic-aerodynamic coupling effects. The number of potential blade-vortex interactions for a given flight condition is directly proportional to the number of rotor blades. The choice of two rotors minimizes the number of encounters and facilitates the interpretation of the time-history of the acoustic pulse. 
The scaling of blade-vortex interaction noise was recently addressed in reference 38. Full-scale data taken by the in-flight method described previously were compared with model-rotor data gathered in an anechoic wind tunnel.

The fu11-scale comparison data were taken on the AH-1S helicopter in 1978 and 1979 in two separate test programs and reported in references 12 and 39. The data are repeatable and clearly define the bladevortex interaction noise phenomena of interest. To optimize the signa1to-noise ratio of BVI impulsive noise, selected conditions of forward velocity and rate of descent were flown with the microphone positioned directly ahead of the helicopter but $30^{\circ}$ below the rotor's tip-path-plane (fig. 66). As discussed, the major advantages of the in-flight technique are the long data records, the absence of ground reflections, and the ability to fly conditions normally associated with terminal-area operations.

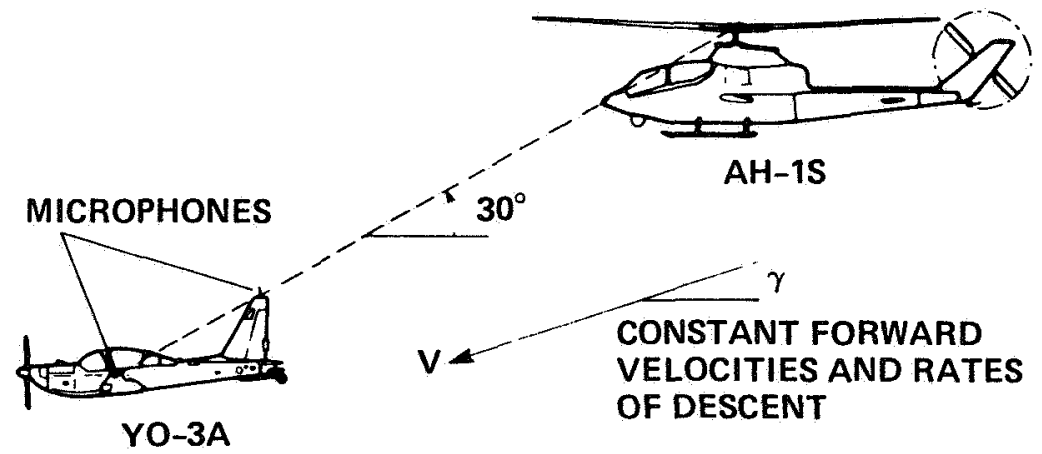

Figure 66.- Ful1-scale blade-vortex interaction acoustic measurement technique.

Model-scale acoustic data were gathered in one of the largest anechoic wind tunnels in the world (CEPRA-19, located near Paris, France). The model rotor was $1 / 7$ geometrically scaled (blade aspect ratio, twist rate, and sectional properties were duplicated) and mounted on a two-bladed teetering hub - as in the full-scale helicopter. A photograph of the rotor installed in CEPRA-19 is shown in figure 67. Dynamic scaling was not attempted. Fortunately, the rigid, large-mass. blades appeared to render the aeroelastic and dynamic effects to a secondary status. Details of the testing procedure are given in reference 38. (There were some small-section geometry differences between the model and full-scale blades. Details can be found in reference 38.)

Besides blade geometry and relative orientation between the rotor and the microphone, there are at least four nondimensional parameters which should be duplicated if model-scale acoustic data are to be expected to match full-scale data. As we have discussed, the in-plane geometry (see fig. 68) between the rotor blade and the tip-vortex 


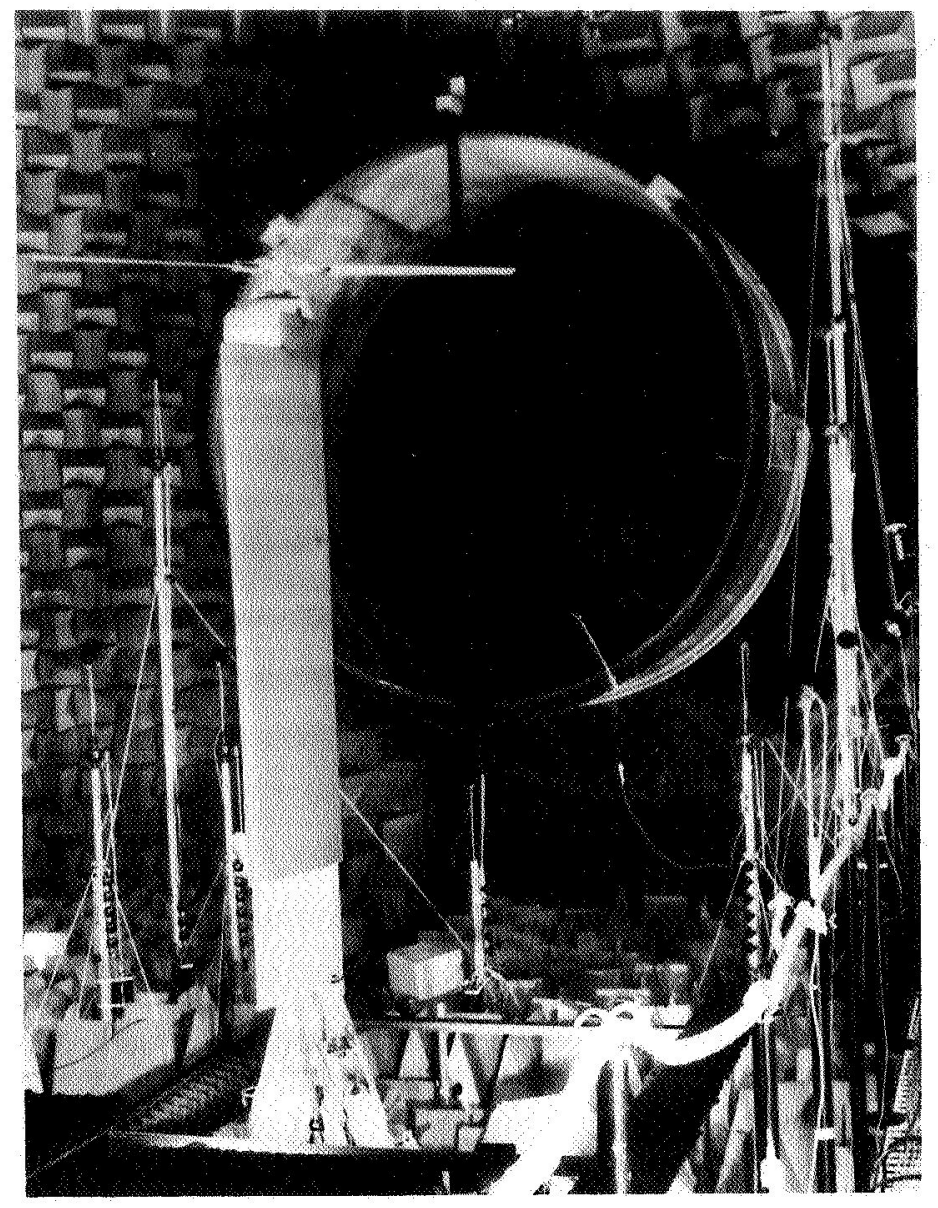

Figure 67.- $1 / 7$ scale model rotor and test stand installed in French CEPRA-19 anechoic wind tunnel.

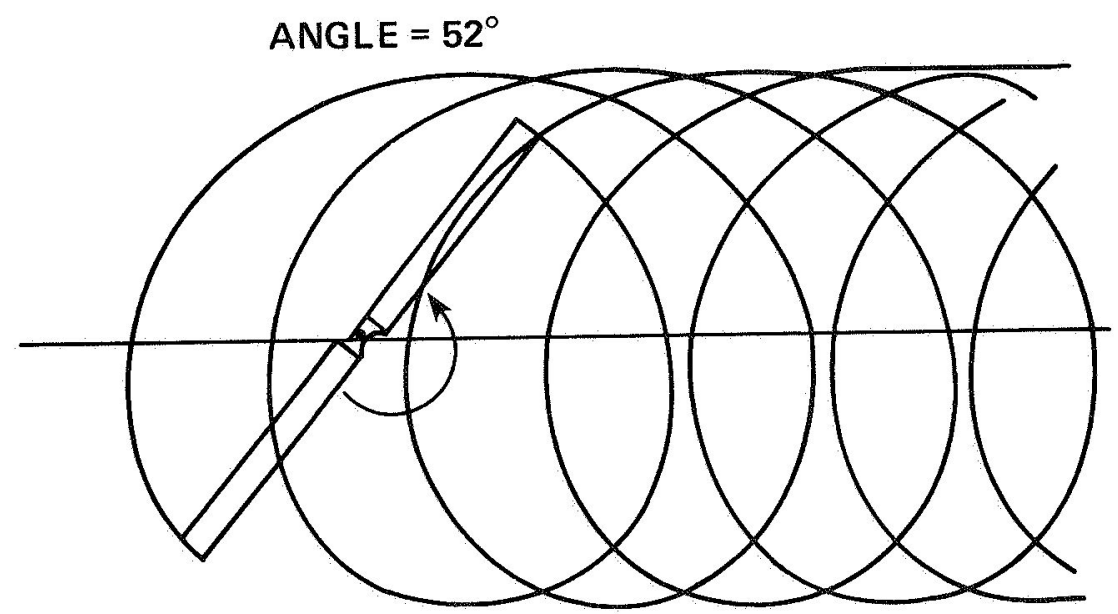

Figure 68.- Epicycloid tip-vortex pattern for $\mu=0.164$.

structure is a dominant BVI interaction parameter. The first nondimensional parameter, advance ratio $\mu$ determines these large-scale geometrical interaction patterns. Advance ratio $\mu$ is defined to be the ratio of the helicopter's forward velocity of translation divided by the main rotor tip-speed, that is, $\mu=V / V_{t i p}$. The in-plane projection of 
the spatial tip-vortex positions is governed by the free-wake geometry of the rotor-wake system. A very good approximation to these geometrical in-plane projections can be made if self-induced wake distortion is totally neglected (rigid wake). In this case, the in-plane projection simply becomes two epicycloid tip-vortex patterns shed from each rotor blade tip, as shown in figure 68. The major differences between this calculation and the one given in figure 68 are in the tip region after the individual vortices begin "rolling together" to form the classical fixed-wing-like vortex system. However, the BVI encounter often occurs before this distortion becomes effective. In these regions above $\mu=0.1$, the rigid and free-wake in-plane projections are almost indistinguishable.

Because advance ratio governs the large-scale BVI geometry, it plays a key role in the acoustic radiation. When viewed from above, the rotor appears to slice through the epicycloid pattern of previously shed tip vortices. The resulting loci of interactions determines the number and strength of the blade-vortex interaction encounters and thus strongly influences the radiated noise.

For scaling purposes, let us assume that only changes in pressure on the surface of the blades causes BVI impulsive noise. As before, the second term of equation (1) becomes

$$
P^{\prime}(x, t)=-\frac{1}{4 \pi} \frac{\partial}{\partial x_{i}} \iint\left[\frac{P_{i j} n_{j}}{r\left[1-M_{r}\right]}\right]_{\tau} d S
$$

where $P_{i j}$ is the local surface-pressure tensor on the surface of the rotor blade. Nondimensionalizing this pressure tensor, we obtain

$$
P^{\prime}(x, t)=-\frac{1}{4 \pi} \frac{\partial}{\partial x_{i}} \iint\left[\frac{{ }^{C_{P}}{ }_{i j} n_{j} \rho_{o} V^{2}}{r\left|1-M_{r}\right|}\right] d S
$$

where

$$
C_{P_{i j}} \equiv \frac{P_{i j}}{\rho_{o} V^{2}}
$$

As in the high-speed scaling arguments, if we assume geometric scaling and introduce the nondimensional unit of time $\bar{t} \equiv t /(2 \pi / \Omega)$, then

$$
\mathrm{d} \overline{\mathrm{S}}=\frac{\mathrm{dS}}{\mathrm{R}^{2}}, \quad \overline{\mathrm{r}}=\frac{\mathrm{r}}{\mathrm{R}}, \quad \overline{\mathrm{x}}=\frac{\mathrm{x}}{\mathrm{R}}, \quad \overline{\mathrm{t}}=\bar{\tau}+\frac{\overline{\mathrm{r}}}{2 \pi} \mathrm{M}_{\mathrm{T}}
$$


and

$$
C_{p}^{\prime}(\bar{x}, \bar{t})=\frac{P^{\prime}(\bar{x}, t)}{\rho_{o} a_{0}^{2}}=\frac{-1}{4 \pi} \frac{\partial}{\partial \bar{x}_{i}} \iint\left[\frac{C_{P_{i j}} n_{j} M^{2}}{\bar{r}\left|I-M_{r}\right|}\right]_{\bar{\tau}} d \bar{S}
$$

where $M=V / a_{0}$. So if rotor, microphone geometry, Mach number, and time are scaled, the acoustic pressure coefficient is uniquely determined. Notice that this result is similar to the high-speed scaling arguments presented earlier. A11 comparisons between model and full-scale results are done in referred acoustic pressure - as was done for the high-speed scaling. ${ }^{2}$ Therefore,

$$
\begin{aligned}
P_{S L}^{\prime}(\bar{x}, \bar{t}) & =\frac{P_{O_{S L}}}{P_{o}} P^{\prime}(\bar{x}, \bar{t}) \\
P_{m_{S L}}^{\prime}(\bar{x}, \bar{t}) & =\frac{P_{o_{m_{S L}}}}{P_{o_{m}}} P_{m}^{\prime}(\bar{x}, \bar{t})
\end{aligned}
$$

These scaling arguments highlight the importance of the second key nondimensional parameter, rotor Mach number. Because the geometry of the problem is scaled and $\mu$ (advance ratio) is also duplicated, choosing the hover tip Mach number $\left(M_{\mathrm{HT}}\right)$ completely specifies the advancing-tip Mach number $\left[\mathrm{M}_{\mathrm{AT}}=(1+\mu) \mathrm{M}_{\mathrm{HT}}\right]$ and the Mach number in the radiation duration, $M_{\mathrm{r}}$. In effect, all Mach numbers associated with the large-scale geometry of BVI are governed by two nondimensional parameters, $\mu$ and $M_{\mathrm{HT}}$.

Judicious matching of the third and fourth nondimensional parameters thrust coefficient, $\mathrm{C}_{T}$, and nondimensional inflow - is necessary to duplicate the pressure coefficients $\left(\mathrm{C}_{\mathrm{p}_{i j}}\right)$ of the mode1 and ful1-scale experiments. For a geometrically scaled rotor, the thrust coefficient governs the local angle of attack of the rotor blade and thus the steady-pressure field. In addition, it affects the average strength of the shed tipvortex, thus directly influencing the unsteady-pressure field as well. The nondimensional inflow $\mu\left(-\alpha_{i}+\alpha_{T P P}\right)$ also affects the magnitude of the unsteady pressures by governing the vertical separation between the vortex and the rotor blade at the time of an encounter. In a rigorous sense, this parameter should scale over the portion of the rotor disk where blade-vortex interactions occur. However, it is of ten assumed that by scaling geometric properties and $C_{T}$, an average value in space and

${ }^{2}$ The full-scale pressure time-history data shown here are from reference 11. The data were corrected for density but not for speed-ofsound effects to an effective pressure at sea level. As mentioned previously, direct comparisons should account for $a_{0}$ changes in both the model and full-scale results. The effect can increase the amplitude disparity between mode1 and full-scale by as much as $6 \%$. 
time of the induced angle $\left(\alpha_{j}\right)$ at the rotor disk governs the interaction problem $\left(\alpha_{i} \sim C_{T} / \mu\right)$. Therefore, if $C_{T}$ and $\mu$ are duplicated in a mode1to full-scale test, the nondimensional inflow or tip-path-plane angle $\left(\alpha_{\text {TPP }}\right)$ becomes the fourth nondimensional test variable.

In norma1, unaccelerated level flight, the helicopter pilot must tilt the rotor tip-path-plane ( $\alpha_{\text {TPP }} \equiv$ the angle between the plane of the rotor tips and the incoming velocity vector; positive for rearward tilt) to balance the drag of the vehicle at each velocity. The result is an increasingly negative tip-path-plane angle with increasing forward velocity. In a climb, the rotor must be tilted farther forward ( $\left.\alpha_{T P P}\right)$ both to balance drag and to oppose gravity, whereas in a descent the rotor must be tilted rearward $(+\alpha$ TPP $)$. The strongest blade-vortex interactions are known to occur in the descent condition, when the tip-pathplane angle is positive, forcing sections of the shed tip-vortices close to or into the rotor's tip-path-plane (fig. 68).

The most rigorous test of the scalability of impulsive noise is the most direct: that of simply comparing the character of the model- and full-scale acoustic time-histories on a one-to-one basis. In addition to being a straightforward comparison, it is also helpful in identifying the occurrences of blade-vortex interactions in the acoustic signatures. This phenomenological approach is illustrated in figure 69 for the AH-1G helicopter for a microphone located approximately $30^{\circ}$ beneath the plane of the rotor tips. This relative orientation of the microphone and the rotor is known to maximize the blade-vortex interaction noise and to reduce the intensity of high-speed impulsive noise. In figure $69(\mathrm{a})$ a measured acoustic time-history is shown for one rotor revolution as measured using the full-scale, in-flight technique. The helicopter and measurement aircraft were flown in formation at a $60 \mathrm{knot}$ (IAS) partialpower descent ( $400 \mathrm{ft} / \mathrm{min}$ rate of descent), a condition known to produce strong blade-vortex interaction noise. The four important nondimensional scaling parameters are listed in figure $69(\mathrm{a})$. At this $30^{\circ}$ microphone position, both the blade-vortex interaction noise and high-speed impulsive noise are discernible. During advancing blade-vortex interaction, a sequence of narrow, small-negative and large-positive spikes occurs in the waveform just before the broader negative-pressure pulse. As discussed previously, the latter is the high-speed thickness noise and is characteristic of acoustic radiation below the delocalization Mach number at a microphone position $30^{\circ}$ under the rotor plane. It should be noted that the fu11-scale data in figure 69(a) show a "snapshot" of a long data record. Time-averaging of the in-flight signals is prohibited by small but discernible changes in the relative position between the microphone and the helicopter.

The model-scale data, at approximately the same scaled geometric distance, are shown in figures 69 (b) and 69 (c). Figure 69(b) presents one instantaneous snapshot of one rotor revolution and figure 69(c) presents an average of 100 revolutions. The similarity of the mode1- and full-scale pulse shapes is evident. 

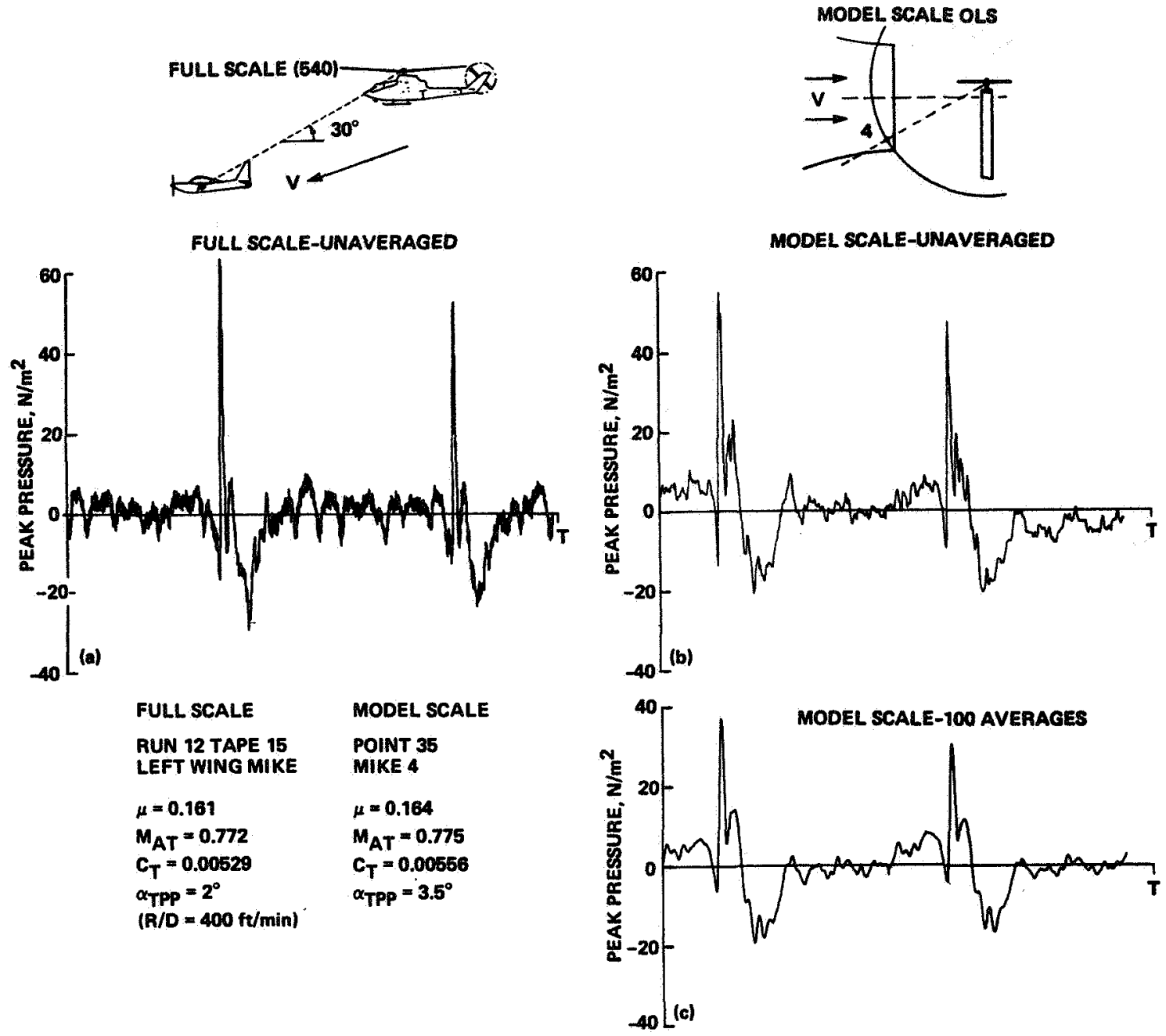

Figure 69.- Time-history comparisons of full-scale and model-scale helicopter blade-vortex interaction noise.

A more complete picture of the comparison of model- and full-scale blade-vortex interaction noise is shown in figure 70 for similar flight conditions. Good general agreement between model-scale and full-scale is apparent at all descent conditions. The averaged mode1-scaled bladevortex interaction amplitudes, as well as pulse shapes, match those of the full-scale data. The figure also shows that the acoustic pulses resulting from a BVI with a young vortex tend to decay, and that those pulses resulting from a BVI with an older vortex tend to grow with increasing descent rate (more positive tip-path-plane tilt). As explained before, as the tip-path-plane of the rotor is tilted rearward, simulating descending flight, a vortex-interaction encounter with an older vortex is more likely. The mode1- and full-scale data substantiate 


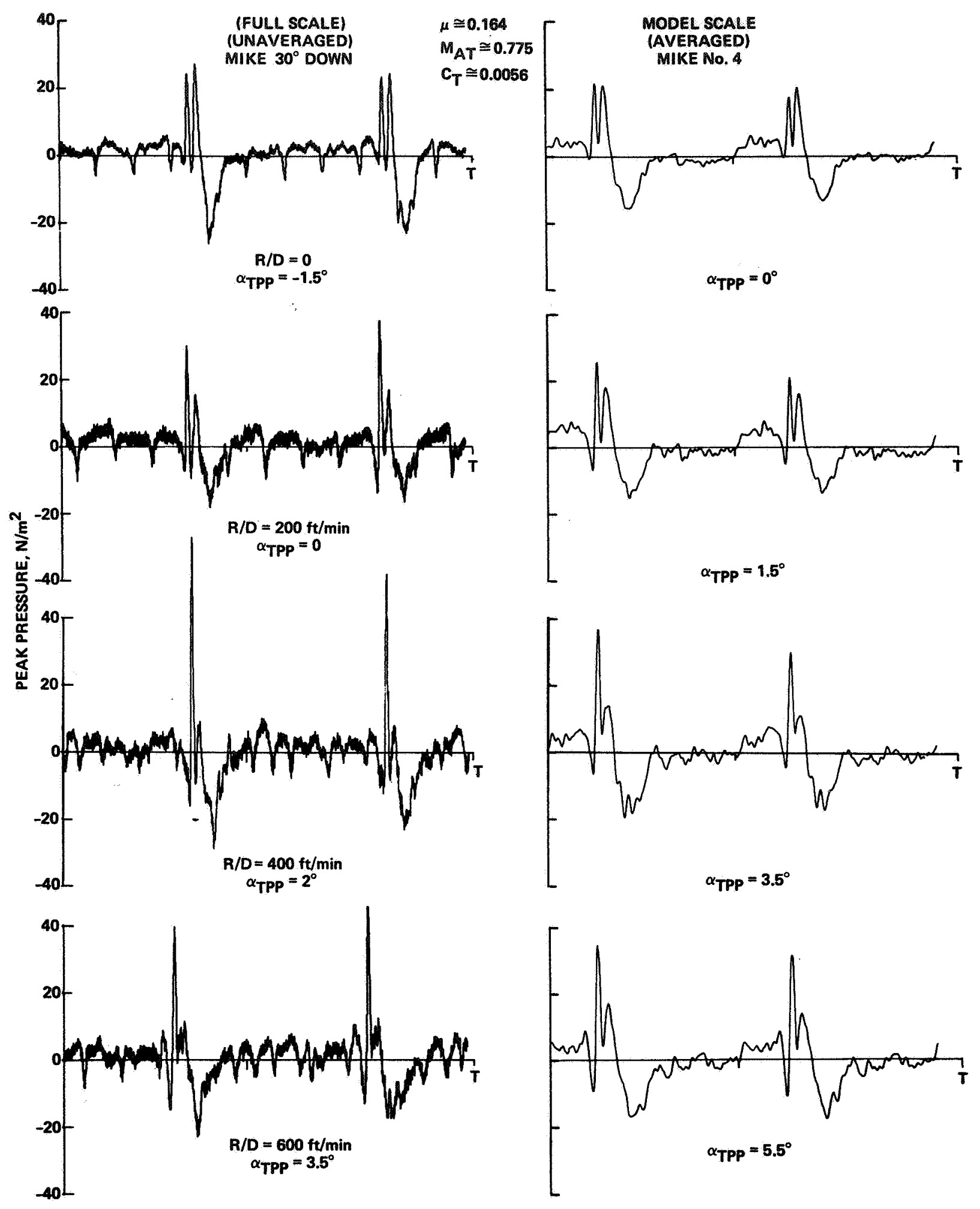

Figure 70.- Time-history comparisons of full-scale and model-scale helicopter blade-vortex interaction noise for several descent conditions. 
this trend and demonstrate the continuity of the blade-vortex interaction process.

These BVI impulsive-noise scaling results are quite encouraging. They demonstrate that it is possible to duplicate many of the features of the ful1-scale. BVI phenomena on model-scale rotors. However, there remain some discrepancies in this first BVI scaling comparison. Most notably, the amplitude of the model-scale BVI is consistently less than that of the full-scale data. In addition, the pulse widths of the model scale seem wider than those of the ful1-scale data. Both discrepancies suggest that the tip-vortex strength of the model may be less intense than the full-scale data. Further analysis of these tests and follow-on testing in the DNW wind tunnel (ref. 14) should help clarify these concerns.

One of the most interesting aspects of the blade-vortex interaction phenomenon is the directivity of the resulting noise. In general, we have shown (fig. 71) that the BVI noise is radiated forward and down approximately $30^{\circ}$ beneath the rotor plan (ref. 12). Figure 71 confirms this fact for the $\mu=0.164$ and $\alpha_{\text {TPP }}=3.5^{\circ}$ case but also indicates that the rotor noise decay is less gradual from the $30^{\circ}$ position toward the rotor's tip-path-plane than farther below this position.

The 1atera1-directivity sweep shown in figure 72 at the $\psi=30^{\circ}$ elevation angle reveals many interesting phenomena as well. The impulsive noise spike is quite weak at the $\theta=90^{\circ}$ position of the microphone and appears to consist of one event. It grows as the microphone is moved to the $45^{\circ}$ position and grows even larger at the $\theta=30^{\circ}$ position. Here it is apparently joined by another pulse. The amplitude of the first pulse increases further at the straight-ahead microphone position and remains high at the microphone retreating side positions. At the $-45^{\circ}$ position, a decrease in amplitude occurs, some of which may be attributable to blockage by the rotor stand.

Some caution should be exercised when interpreting the data recorded on the microphone located near the $3-\mathrm{m}$ nozzle. The reverberant/reflected field could have distorted the results somewhat. Shear-layer effects can also be important at these microphone locations because the sound path enters the shear layer at shallow angles. Nevertheless, the general trends illustrated in figures 71 and 72 are believed to be correct.

One of the best ful1-scale experimental attempts to document the aerodynamic flow field encountered by a helicopter rotor was the joint U.S. Army/Be1I Helicopter Textron flight-test program called the "Operationa1 Loads Survey (OLS)" (refs. 40 and 41). In-flight aerodynamic, structural, and acoustic data were gathered throughout a wide range of operating conditions. The instrumented helicopter was an AH-1 Cobra, quite similar to the one used to gather the in-flight acoustic data discussed in the previous section. One minor difference is that the Operational Loads Survey blade sections were modified to incorporate extensive 


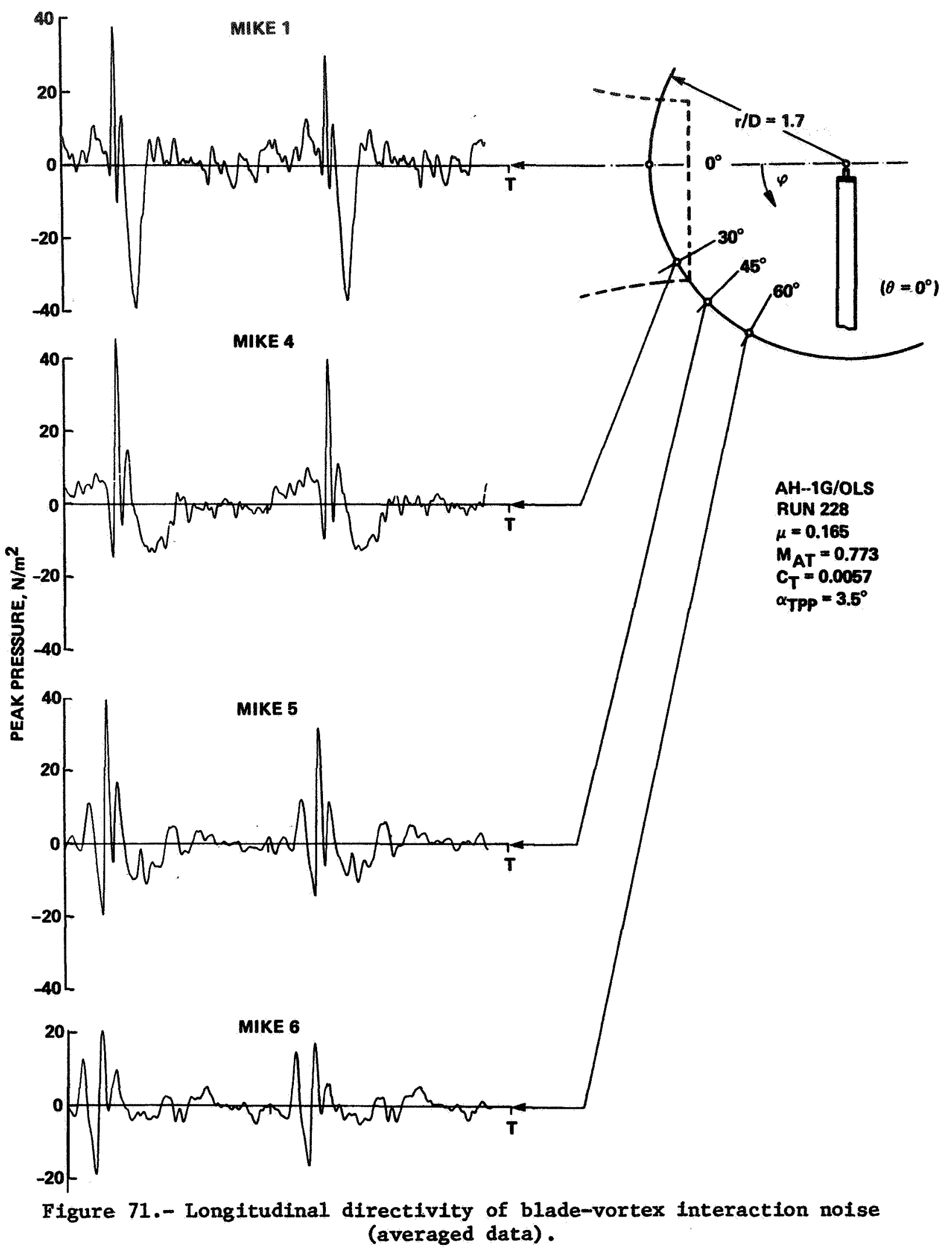




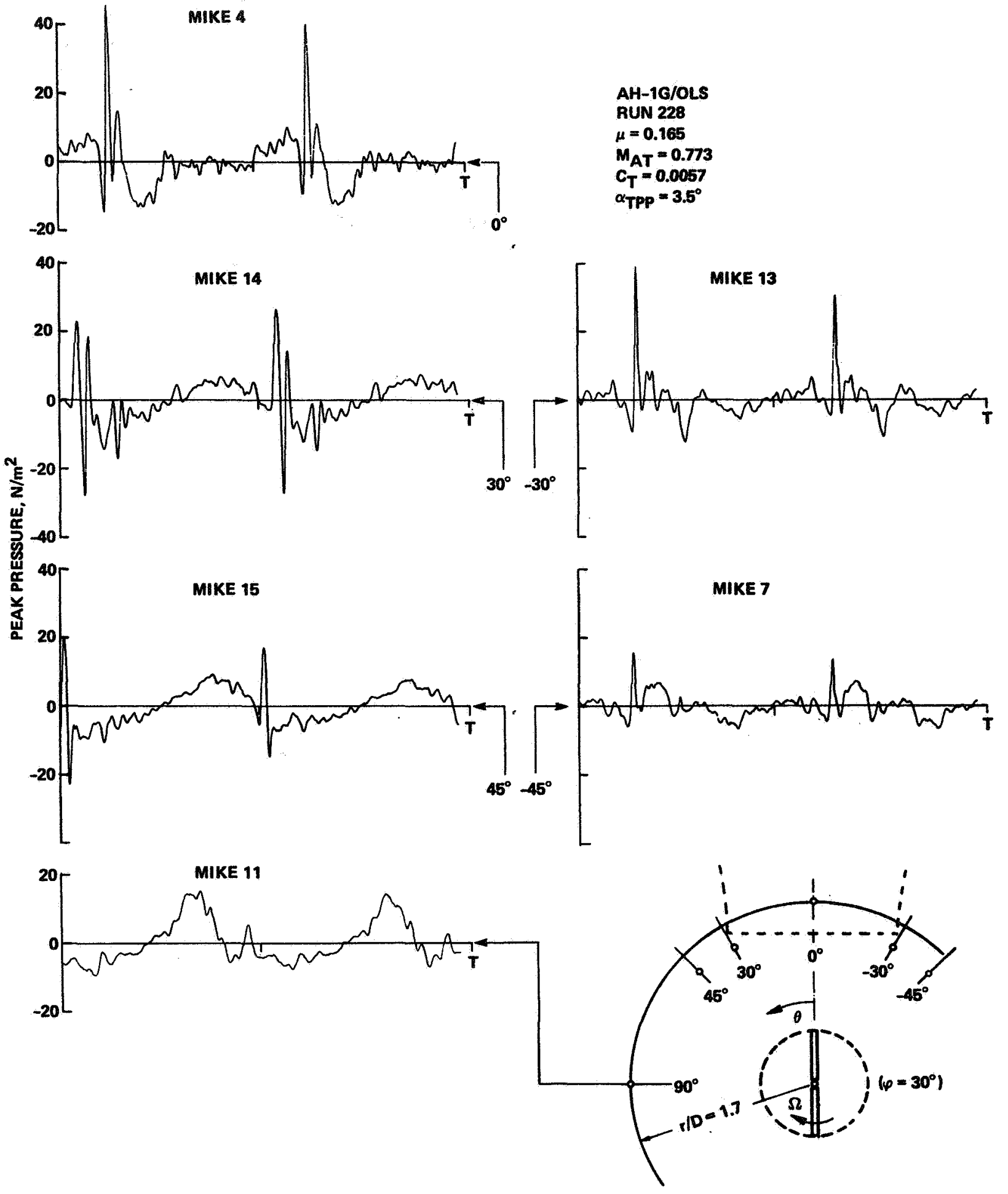

Figure 72.- Lateral directivity of blade-vortex interaction notse (averaged data, $\phi=30^{\circ}$ ). 
rotor-blade pressure instrumentation. Mounted on the upper and lower airfoil surfaces were $110 \mathrm{Kulite}$ absolute-pressure transducers to measure the static-pressure distribution over the airfoil at five spanwise stations. The blade-pressure measurement system had a frequency response of $400 \mathrm{~Hz}$. Thus, events occurring within approximately $5^{\circ}$ steps in rotor azimuth were measured. As shown in figure 73, in-flight microphones were also used to measure simultaneously the near-field noise at three fixed positions. Additional experimental details can be found in references 41 and 42 .

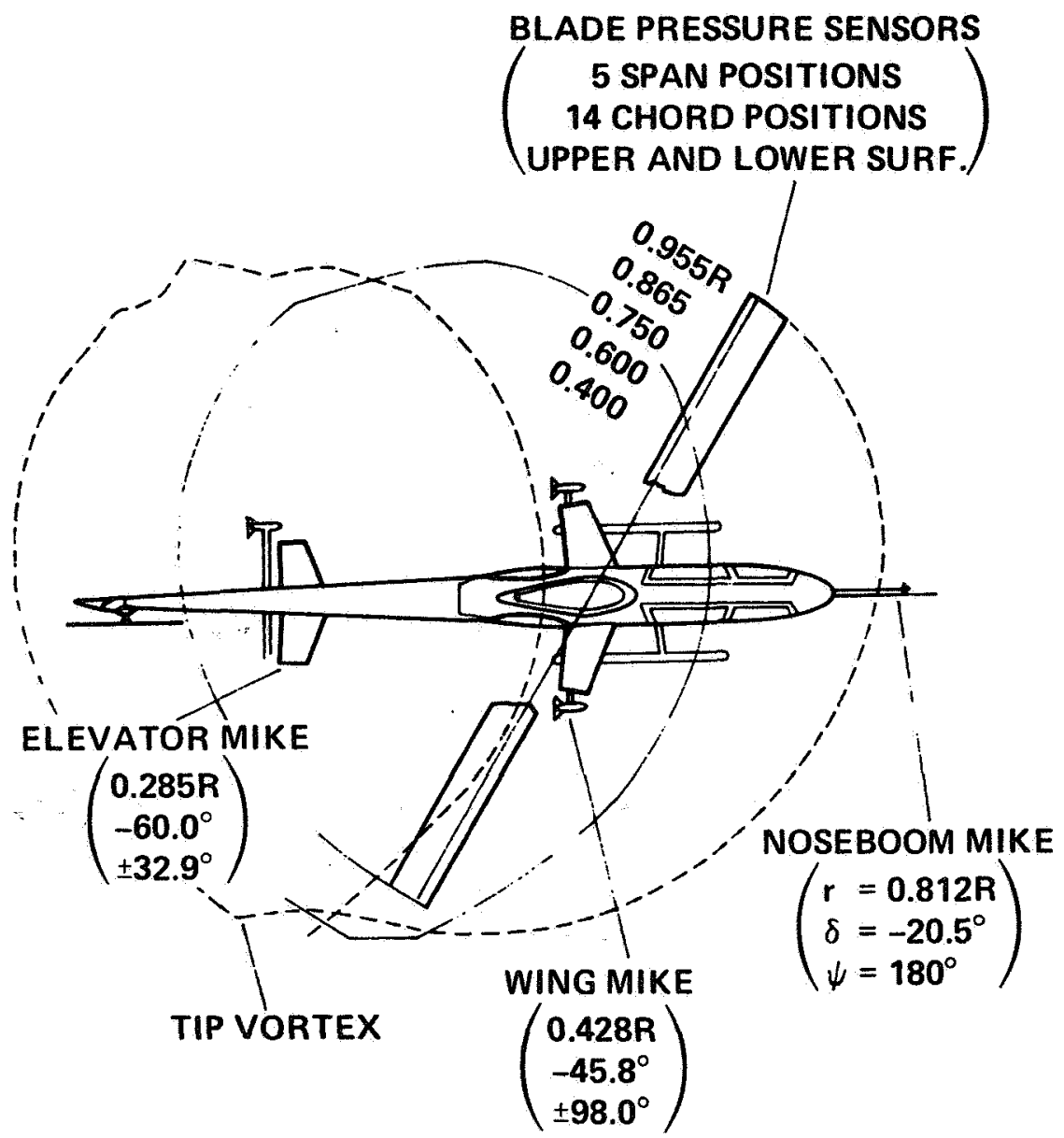

Figure 73.- Simultaneous measurement of blade surface-pressure and noise.

Figures 74 and 75 present an example of the measured pressure fluctuations for a typical BVI descent case $(\mu=0.147$, rate of descent $=200 \mathrm{ft} / \mathrm{min}$ ) at several spanwise and chordwise stations, respectively. The leading-edge pressure transducers (fig. 74) at several spanwise locations near the blade tip identify the aerodynamic BVI phenomena quite nicely. Rapid pressure fluctuations from $\psi \approx 45^{\circ}$ 


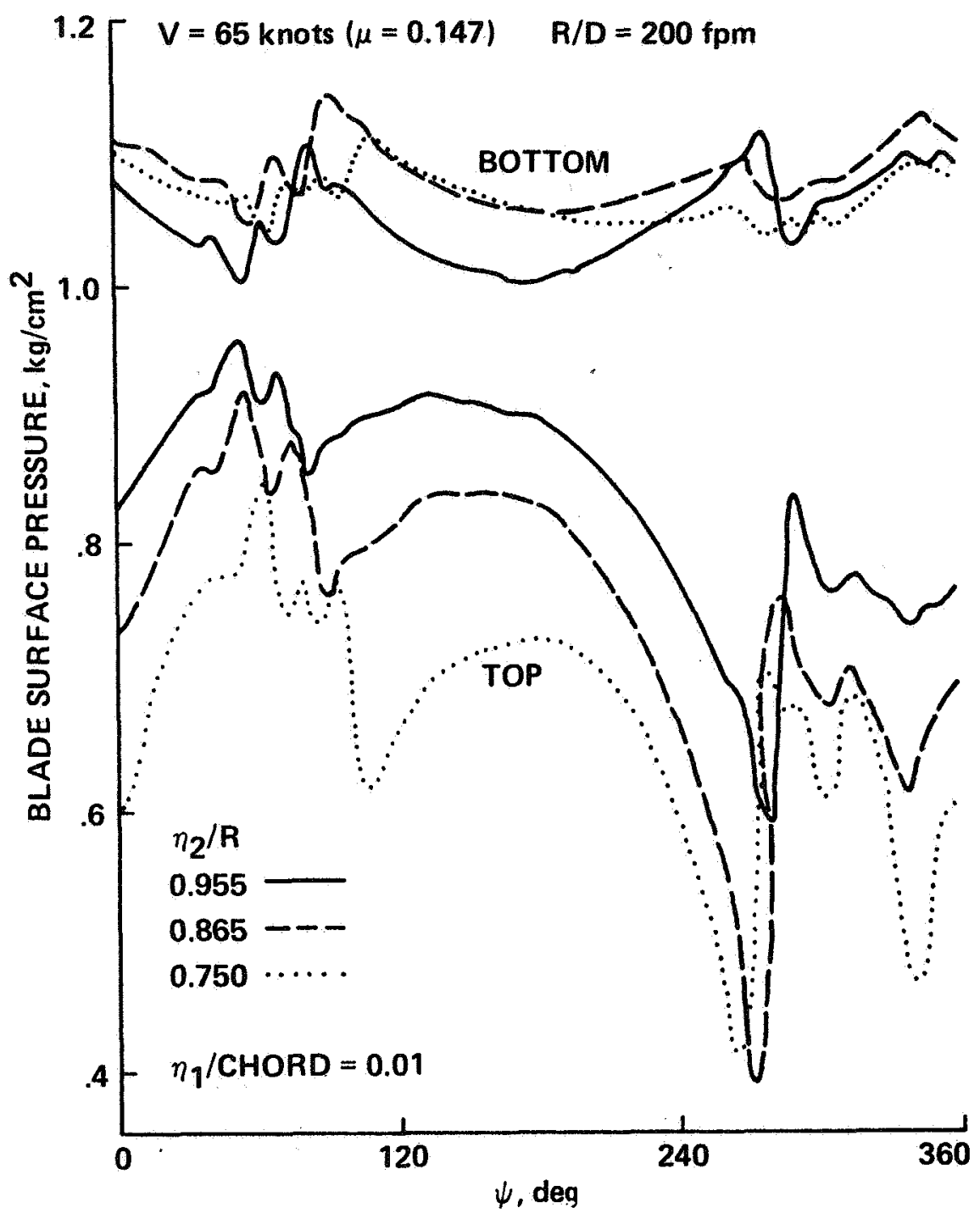

Figure 74.- Example of blade surface-pressure history at different span positions (ref. 40).

to $\psi \approx 90^{\circ}$ are seen corresponding to the BVI locations previously noted on the advancing side of the rotor disk. Relatively strong pressure fluctuations are also seen on the retreating side $\left(\psi \approx 270^{\circ}\right)$. These also generally correlate with the BVI locations indicated in the top view of the free-wake sketched in figure 68. Unfortunately, the 400-Hz upper frequency limit on the blade pressure transducers apparently tends to smooth the pressure variations that are shown. More recently (ref. 38) similar model-scale tests of a scaled ols rotor have confirmed these general findings and have indicated that the actual BVI pressure transducer response does contain higher frequency data. 


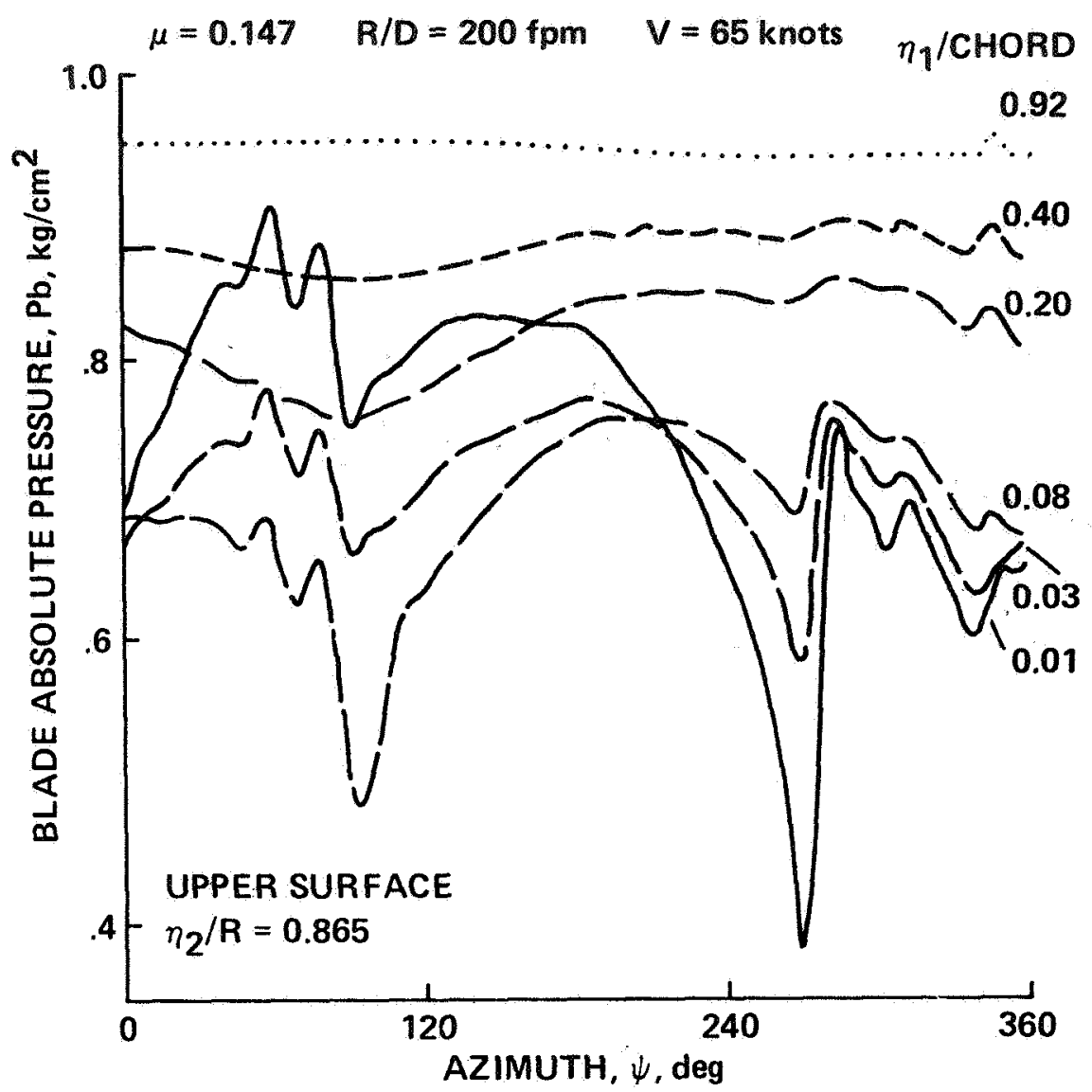

Figure 75.- Example of blade surface-pressure history at different chord positions (ref. 40).

One of the most important findings of this experiment is shown in figure 75 where the upper-surface pressure transducer responses are plotted versus azimuth angle for several chordwise stations. The BVI phenomenon is seen to be concentrated near the very leading edge of the blade chord. In fact, the dominant BVI pressure changes are confined to the first $10 \%$ of the blade chord. At $20 \%$ of the chord, very few BVI pressure changes are noted. The same pattern (see ref. 41) also exists for the lower-surface pressure transducers. In the more recent mode1scale tests (refs. 14 and 38), these findings were substantiated, indicating that the aerodynamic source location for BVI noise is the very leading edge of the rotor blade. It was also noticed that the pressure disturbances on these model-rotor tests were "shock-like," again indicating that the aerodynamic phenomena near the rotor's leading edge were quite sharp (nearly discontinuous). These experiments clearly show that the BVI is an impulsive-like phenomenon located near the leading edge of the rotor blade. 
Simultaneous in-flight near-field acoustic measurements were also recorded during these experiments. As expected, the advancing-blade/ vortex interactions radiated most of their energy forward and were measured on the right wing-tip and nose-boom microphones. The retreatingblade/vortex interactions radiated to aft directions and were measured on the left wing tip and elevator microphones. The largest acoustic pulses were recorded on the right-wing-tip and nose-boom microphones, even though figure 74 shows that the advancing-blade interaction pressure variations are less than those measured on the retreating side. The conclusion to be drawn is that advancing-side BVIs are apparently "acoustically" more efficient than the retreating side BVIs.

The near-discontinuous nature of these measured pressure variations is supported by some earlier Schlierin photographs of advancing-side BVI taken by Tangler ( $r e f .9$ ) on a small model rotor in a wind tunnel. The photographs shown in figure 76 are admittedly qualitative in many testing respects, because parameters such as thrust, blade design, and tip-pathplane tilt were adjusted to obtain clear photographs. In addition, the Schlierin system presents only two-dimensional representations of a

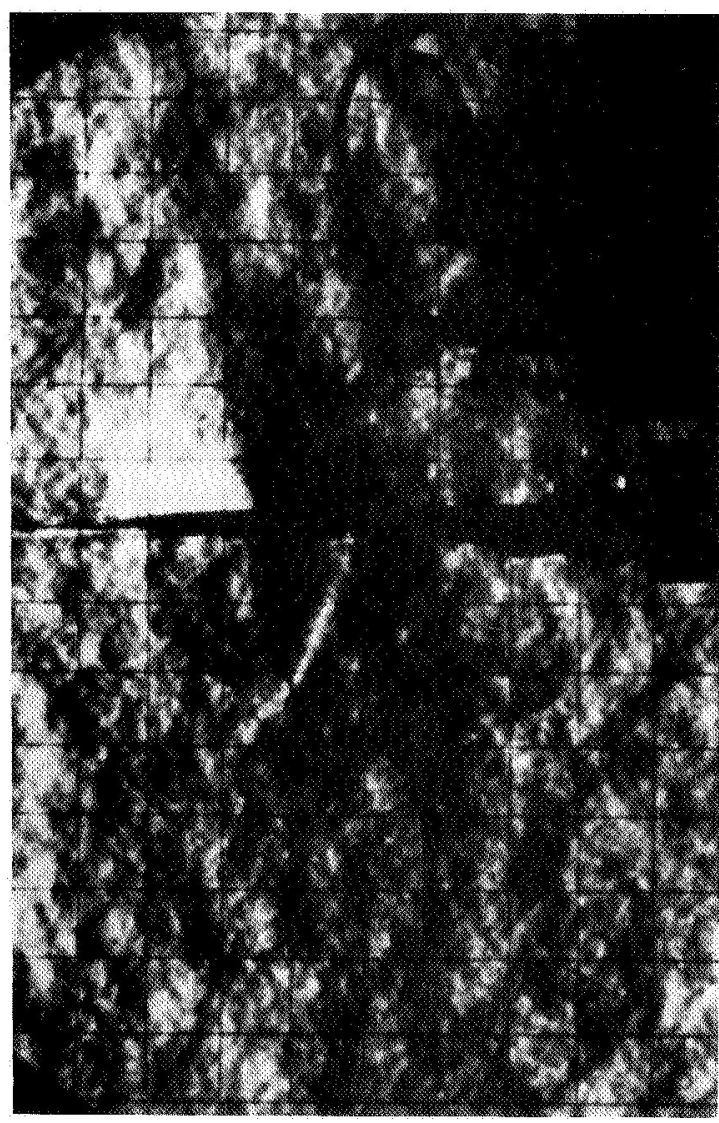

$\psi=70^{\circ}$

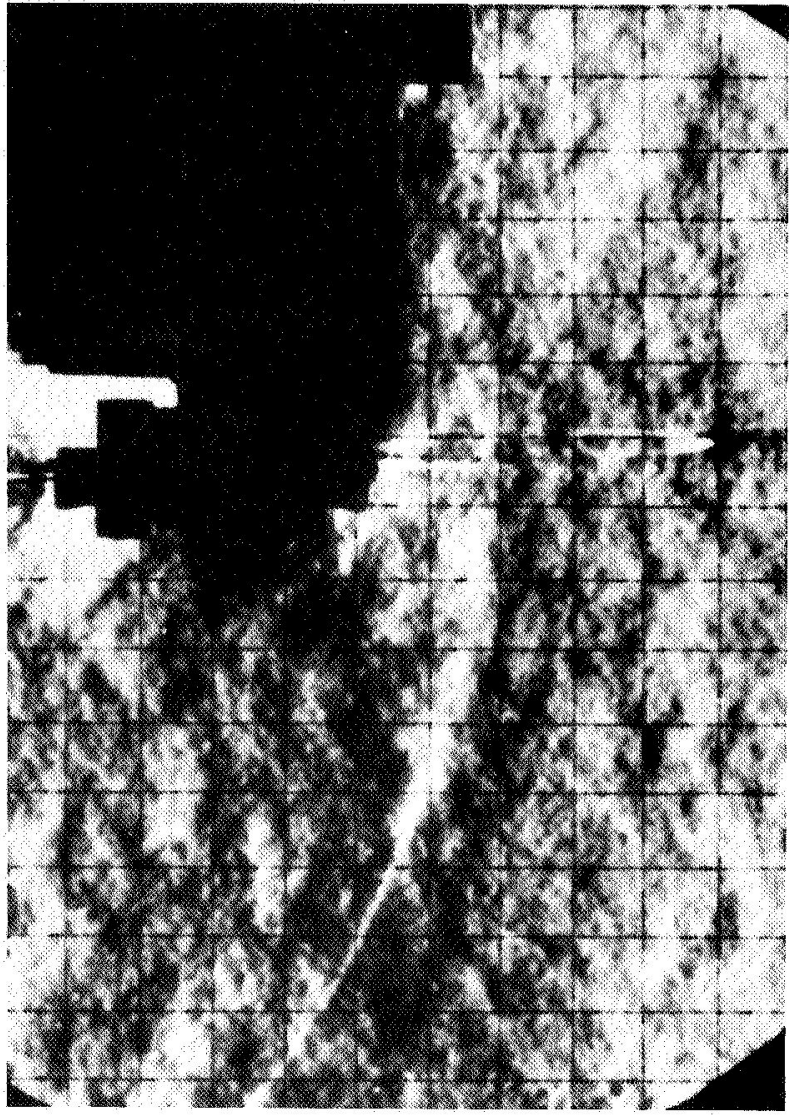

$\psi=90^{\circ}$

Figure 76.- Shock propagation from blade-vortex interaction. 
three-dimensional phenomenon. Nevertheless, the BVI interaction shown is believed to be the $11 / 2$ old (No. 3) "near broadside" encounter illustrated in figure 76 which in a local sense is nearly two dimensional. A lower-surface shock wave is seen in these photographs which begins near the blade leading edge and propagates forward and downward into the direction of the oncoming flow. Judging from these remarkable photographs, the compressible nature of the BVI phenomena at these typical full-scale tip Mach numbers is clear. The photographs also support the full-scale and model-scale experimental findings that the aerodynamic source location is the leading edge of the rotor blade.

It is tempting to conclude that shock waves play a role in many of the blade-vortex interaction encounters on full-scale helicopters. However, the full-scale experimental evidence to support such a conclusion is sketchy and inconclusive at best. The measured full-scale in-flight acoustic data (ref. 11) have shown that the waveform exhibits a "shocklike" character. However, a discontinuous radiating shock wave similar to high-speed delocalization has never been quantified either in groundbased or in-flight acoustic measurements. What seems certain is that compressibility plays a significant role in the formation of the sharp pressure disturbance. On full-scale helicopter measurements to date, these disturbances radiate to the acoustic far-field as saw-tooth waves.

It has always been the acoustician's dream to be given accurate pressure measurements on the surface of the rotor blade and then to be asked to compute the acoustic pressure at some remote microphone 1ocation. Theoretically, equation (3) can be used to sum pressure disturbances to calculate the acoustic far-field. Such calculations were performed by Nakamura (ref. 10) utilizing the full-scale ols data with remarkably good results. However, these results were achieved after many of the experimentally observed phenomena previously described were carefully accounted for in the analysis.

The first assumption in this analysis was that only known surfacepressure variations contributed to the radiated noise (eq. (3)). of course, the noncompact nature of the aerodynamic and acoustic problems was realized and included in the acoustic formulation. However, the first attempt at just "plugging in" all of the interpolated blade-vortex interaction pressures totally failed. The acoustic prediction only accounted for the very low frequency loading noise. Al1 higher frequency data were inadvertently filtered by the necessary interpolation between data points (ref. 10). The important lesson to be learned from this exercise is that just "plugging in" measured or calculated pressures to an existing computer code to calculate far-field acoustics is probably a waste of time unless the phenomenon of interest is carefully represented in the input data.

Representation of the BVI phenomena from the measured data was not a trivial task. A computer model was constructed which carefully fit the measured data and kept track of the interaction loci. In fixed-space 
coordinates, these interaction loci determine the "trace Mach number" and thus govern the relative efficiency of the BVI. Figure 77 from reference 10 shows some of the difficulties of constructing continuous BVI interaction loci from the measured data. A11 of the expected BVI pressure pulses were not measured at each radial station. Instead, it appeared as if the blade-tip region encountered fewer interactions on the advancing side than did the more inboard regions. These findings are probably a result of the nonplanar character of the BVI and are aggravated by the $400-\mathrm{Hz}$ upper frequency limit of the pressure instrumentation. The intelligent estimates of the interaction structure (shown in fig. 77) were necessary to arrive at a successful BVI model.

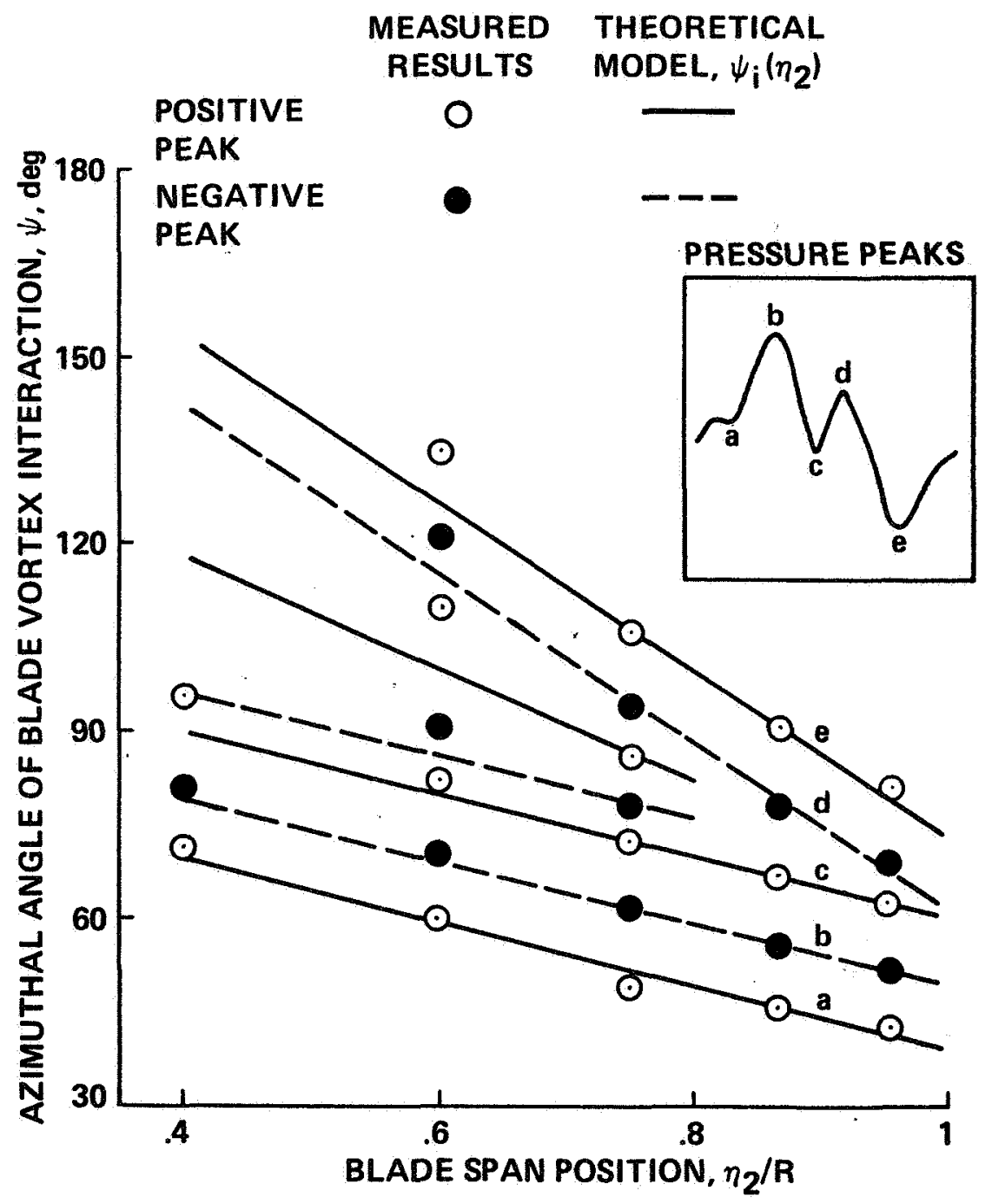

Figure 77.- Modeling of blade-vortex interaction lines from measured results. 
Figure 78 shows the comparison of the measured OLS and computed acoustic waveforms for the right-wing microphone and nose-boom microphone. The general waveform, including the impulsive shape, is well predicted in both observer positions; however, the peak amplitudes are underestimated and the pulse widths overestimated. At the present time it is not known how much of this discrepancy is due to experimental inaccuracies, to numerical interpolation of the measured data, or to the omission of nonlinear terms in the governing equation (1). The wider pulse shapes of the theoretical predictions seem to suggest that the measured pressures did not have a high enough frequency response. New data recently taken on model-scale oLS blades are currently being analyzed to address these concerns.

Based on the mode1- and ful1-scale experiments discussed so far, it is strongly suspected that compressibility plays a role in the aerodynamics

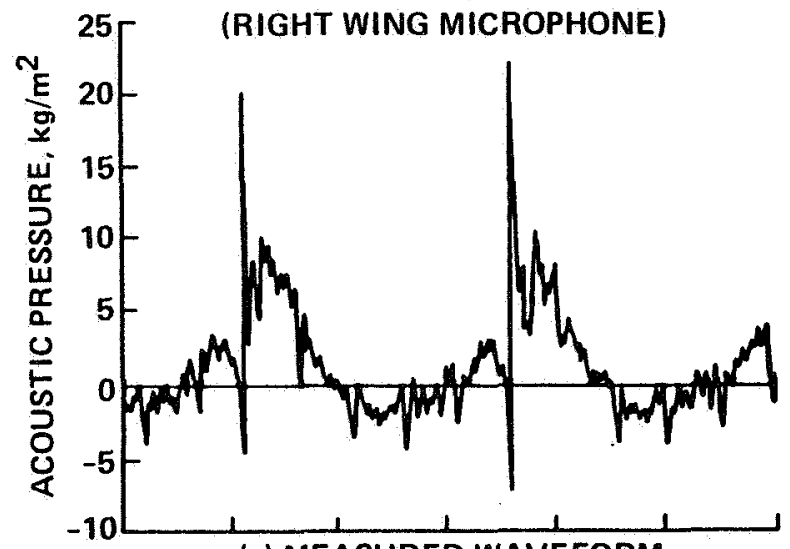

(a) MEASURED WAVEFORM

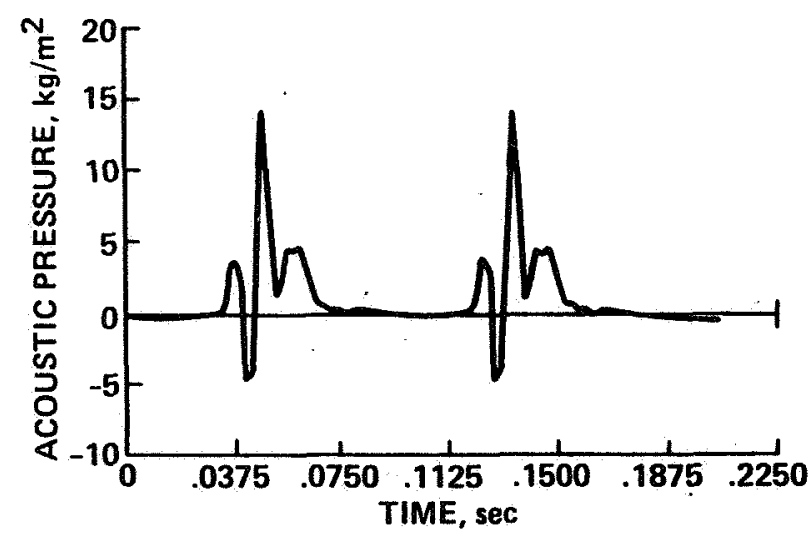

(b) COMPUTED WAVEFORM

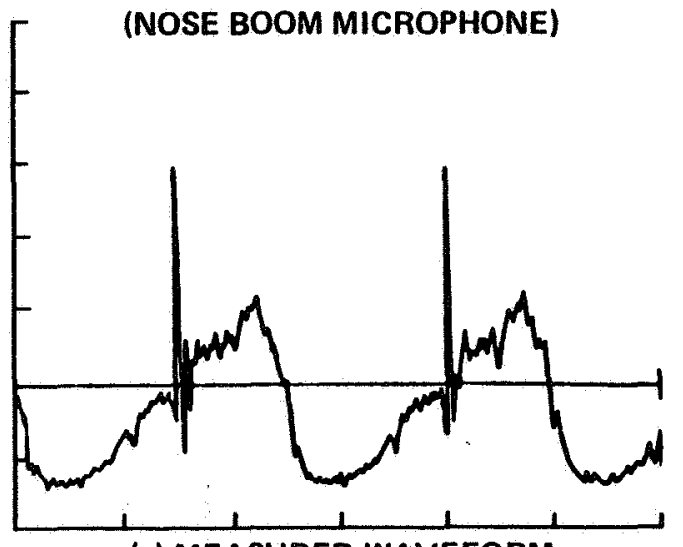

(a) MEASURED WAVEFORM

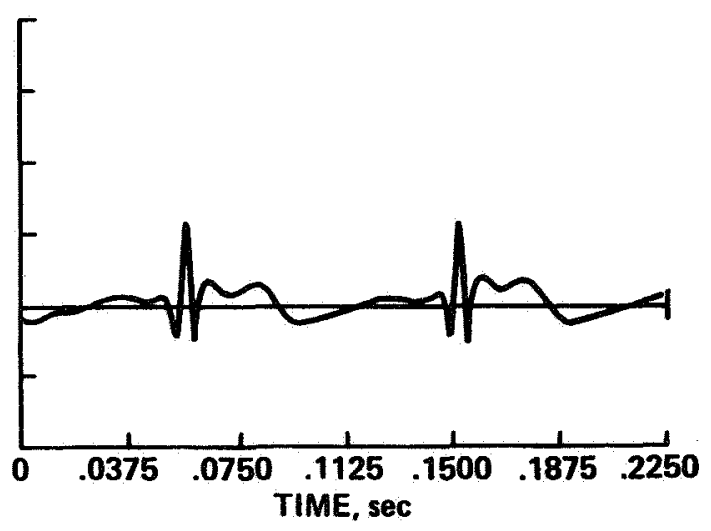

(b) COMPUTED WAVEFORM

Figure 78.- Comparison of acoustic waveform between measurement and computation. 
of BVI. For the near "broadside" BVI encounter depicted in figure 8, the mean-flow velocity $(\omega R+V$ sin $\alpha)$ plus the local velocity induced by the vortex $\left(v_{i}\right)$ can exceed sonic velocities over small regions near the leading edge of the rotor blade. Unfortunately, this local aerodynamic flow field is transonic and unsteady and thus governed by nonlinear equations. As in the high-speed problem, numerical solution of the governing equations is the only known method of capturing these effects.

At the present time, the local transonic flow field which exists during BVI is being addressed by several researchers. The problem has initially been simplified to a two-dimensional encounter with the vortex passing by a stationary airfoil. The governing equations are then numerically solved to yield the aerodynamic, as well as the radiated, noise. A first attempt at such a simulation of BVI was recently performed in reference 42. Even though only the vertical upwash disturbance of the BVI encounter was considered, these preliminary results are encouraging. They reaffirm the importance of leading-edge blade geometry (refs. 9 and 10). When developed, they should be able to suggest leading-edge geometries that tend to minimize the airfoil pressure disturbances that can radiate to the acoustic far-field. Experimental work to support these efforts is also under way.

As mentioned previously, the BVI impulsive-noise phenomena are not as well understood as high-speed impulsive noise. Nevertheless, substantial progress is being made. Several areas in which improvements can and are being made (in some cases) are suggested in the following paragraphs.

The first attempt at scaling BVI impulsive noise (ref. 38), although generally good, is not completely satisfactory. There remain the problems of reduced amplitude and wider pulse shapes of the model scale data. Fortunately, more recent tests (ref. 14) have documented BVI under more controlled aerodynamic and acoustic conditions. The detailed analysis of these data (both blade pressure and radiated noise) should more fully answer the scaling question.

It would also be helpful to conduct some new experiments. In particular, laser velocity measurements on mode1- and full-scale rotors to define the vortex strength and its geometry during a BVI encounter at full-scale tip speeds are suggested.

Another very important question that needs to be addressed is: How applicable are these normal operating tip-speed results to lower tip speeds? From our experience, it appears that the mechanisms of the problems that we have discussed exist on full-scale helicopters with hover tip Mach numbers of 0.6 to 0.73 . However, much of the model-scale BVI experiments that have been run are at lower Mach numbers (refs. 43-45). 
How do these lower-Mach-number results relate to the phenomena which we have been discussing? New testing over a wide range of Mach numbers is needed.

The BVI research to date has focused on a very limited amount of measured acoustic data. In this summary, it has been shown that there are directions in which BVI impulsive noise is efficiently radiated. It remains to calculate these radiation directions and to correlate these findings with high-quality pressure-instrumented rotor blades run in an anechoic wind tunnel. The same high-quality pressure data could be used in equation (3) (similar to that used in ref. 10) to predict the radiated noise.

\section{ACKNOWLEDGMENTS}

The support and contributions of our many friends and colleagues at the Aeromechanics Laboratory are gratefully acknowledged. The inspiration and support of $\mathrm{Mr}$. H. Andrew Morse and Dr. Irving C. Statler, the technical contributions of $\mathrm{Mr}$. Donald Boxwell and $\mathrm{Mr}$. C. Rande Vause, and the experimental support of Mrs. Georgene Laub and Mr. Robert George made the entire effort over the past 10 years possible. In addition, colleagues at the Research and Technology Laboratories, various universities and colleges, the helicopter manufacturers, the European and United States Army Research offices, ONERA, DNW, DFVLR, NLR, and NASA a1so helped with direct as well as indirect support - bringing the talents of many researchers all over the world to bear on the problem of helicopter impulsive noise. 


\section{REFERENCES}

1. Lowson, M. V. (1973): Helicopter Noise: Analysis-Prediction and Methods of Reduction. AGARD Report LS -63 .

2. George, A. R. (1977): Helicopter Noise - State of the Art. AIAA Paper 77-1337, Atlanta, Ga.

3. White, R. P. (1980): The Status of Rotor Noise Technology. J. Am. Helicopter Soc., vol. 25, no. 1, Jan.

4. Ffowcs Williams, J. E.; and Hawkings, D. L. (1969): Philos. Trans. R. Roc. London, Ser. A, vo1. 264, May 8, pp. 321-342.

5. Hawkings, D. L.; and Lowson, M. V. (1974): Theory of Open Supersonic Rotor Noise. J. Sound Vibration, vol. 36, no. 1.

6. Farassat, F. (1974): The Acoustic Far-Field of Rigid Bodies in Arbitrary Motion. J. Sound Vibration, vol. 32, pp. 387-405.

7. Isom, M. P. (1975): The Theory of Sound Radiated by a Hovering Transonic Helicopter Blade. Poly-AE/AM Report No. 75-4, Department of Aerospace Engineering and Applied Mechanics, Polytechnic Inst. of New York.

8. Schmitz, F. H.; and Yu, Y. H. (1979): Theoretical Modeling of HighSpeed Helicopter Impulsive Noise. J. Am. Helicopter Soc., vo1. 24 , no. 1 .

9. Tangler, J. L. (1977): Schlieren and Noise Studies of Rotors in Forward Flight. Paper 77, 33-05, 33rd Annual National Forum of the American Helicopter Society, Washington, D.C.

10. Nakamura, Y. (1981): Prediction of Blade-Vortex Interaction Noise from Measured Blade Pressure. Paper 32, Seventh European Rotorcraft and Powered Lift Aircraft Forum, Garmisch-Partenkirchen, Federal Republic of Germany. .

11. Schmitz, F. H.; and Boxwe11, D. A. (1976): In-F1ight Far-Field Measurement of Helicopter Impulsive Noise. J. Am. Helicopter Soc., vol. 21, no. 4, Oct.

12. Boxwel1, D. A.; and Schmitz, F. H. (1982): Fu11-Scale Measurements of Blade-Vortex Interaction Noise. J. Am. Helicopter Soc., vol. 27 , no. 4 , Oct.

13. Schmitz, F. H.; Boxwe11, D. A.; and Vause, C. R. (1977): HighSpeed Helicopter Impulsive Noise. J. Am. Helicopter Soc., vol. 22, no. 4, Oct. 
14. Splettstoesser, W. R.; Schultz, K. J.; Schmitz, F. H.; and Boxwell, D. A. (1983): Model Rotor High-Speed Impulsive Noise - Parametric Variations and Full-Scale Comparisons. Paper 83-39-53, 39th Annual Forum of the American Helicopter Society.

15. Boxwell, D. A.; Yu, Y. H.; and Schmitz, F. H. (1978): Hovering Impulsive Noise: Some Measured and Calculated Results. NASA CP-2052, 1978, and Vertica, vol. 3, no. 1, 1979.

16. Farassat, F.; Nystrom, P. A.; and Morris, C. E. K., Jr. (1979): A Comparison of Linear Acoustic Theory with Experimental Noise Data for a Small Scale Hovering Rotor. AIAA Paper 79-0608, Seattle, Wash.

17. Farassat, F.; and Martin, R. M. (1983): A Note on the Tip Noise of Rotating Blades. J. Sound Vibration, vol. 86, no. 3, pp. 449-453.

18. Kittleson, J. K. (1983): A Holographic Interferometry Technique for Measuring Transonic Flow near a Rotor Blade. Paper B, Ninth European Rotorcraft Forum, Sept. 13-15, 1983.

19. Isom, M. P. (1979): Some Nonlinear Problems in Transonic Helicopter Acoustics. Report No. 79-19, Department of Mechanical and Aerospace Engineering, Polytechnic Inst. of New York.

20. Schmitz, F. H.; and Yu, Y. H. (1981): Transonic Rotor Noise Theoretical and Experimental Comparisons. Vertica, vol. 5, pp. 55-74.

21. Hawkings, D. (1979): Noise Generation by Transonic Open Rotors. Research Report, Westland Helicopter Limited, June 22.

22. Yu, Y. H.; Caradonna, F. X.; and Schmitz, F. H. (1978): The Influence of the Transonic Flow Field on High-Speed Helicopter Impulsive Noise. Paper 58, 4th European Rotorcraft and Powered Lift Aircraft Forum, Italy.

23. Caradonna, F. X.; and Isom, M. P. (1976): Numerical Calculations of Unsteady Transonic Potential Flow over Helicopter Rotor Blades. AIAA J., vol. 14, no. 4, pp. 482-488.

24. Caradonna, F. X. (1978): The Transonic Flow on a Helicopter Rotor. Ph.D. dissertation, Stanford U., Stanford, Calif.

25. Isom, M. P. (1980): Acoustic Shock Waves Generated by a Transonic Helicopter Blade. Paper 63, 36th Annual National Forum of the American Helicopter Society.

26. Caradonna, F. X.; and Steger, J. L. (1980): Implicit Potential Methods for the Solution of Transonic Rotor Flows. Presented at the 1980 Army Numerical Analysis and Computers Conference, Moffett Field, Calif. 
27. Tauber, M. E.; and Hicks, R. M. (1980): Computerized ThreeDimensiona1 Aerodynamic Design of a Lifting Rotor Blade. Proceedings of the $36 \mathrm{th}$ Annual National Forum of the American Helicopter Society, Washington, D.C.

28. Chattot, J. J. (1980): Calculation of Three-Dimensional Unsteady Transonic Flows Past Helicopter Blades. NASA TP-1721.

29. Shenoy, K. R. (1982): Semiempirica1 High-Speed Rotor Noise Prediction Technique. Presented at the 38 th Annual National Forum of the American Helicopter Society, Anaheim, Calif.

30. Hanson, D. B.; and Fink, M. R. (1978): The Importance of Quadrupole Sources in Predictions of Transonic Tip Speed Propeller Noise. Spring Meeting of the Institute of Acoustics, Cambridge U., England.

31. Rutherford, J. (1983): The Aerodynamics and Aeroacoustics of Rotating Transonic Flow Fields. Paper A-83-39-50-D000, 39th Annual National Forum of the American Helicopter Society.

32. Thibert, J. J.; and Philippe, J. J. (1982): Studies of Aerofoils and Blade Tips for Helicopters. Presented at AGARD/FDP Meeting on Prediction of Aerodynamic Loads on Rotorcraft, London.

33. Kittleson, J.; and Yu, Y. (1982): Holographic Interferometry Technique for Rotary Wing Aerodynamics and Noise. Proc. of 1982 Army Science Conference, vol. II, pp. 209-222.

34. Hanson, D. B. (1976): Near-Field Noise of High Tip Speed Propellors in Forward F1ight. Paper 76-565, 3rd Aero-Acoustics Conference, July.

35. Nakamura, Y.; and Azuma, A. (1978): Improved Methods for Calculating the Thickness Noise. NASA CP-2052.

36. Sternfield, H.; Bobo, C.; Carmichael, D.; Fukushima, T.; and Spencer, R. (1972): An Investigation of Noise Generation on a Hovering Rotor. Part II. Report D210-10550-1, The Boeing Company, Vertol Div., Philadelphia, Penn.

37. Sternfeld, H.; and Schaeffer, E. (1982): An Investigation of Rotor Harmonic Noise by the Use of Small Scale Wind Tunne1 Models. NASA CR-166, 338 .

38. Schmitz, F. H.; Boxwe11, D. A.; Lewy, S.; and Dahan, C. (1982): A Note on the General Scaling of Helicopter Blade-Vortex Interaction Noise. Presented at the 38th Annual National Forum of the American Helicopter Society, Anaheim, Calif. 
39. Boxwe11, D. A.; and Schmitz, F. H. (1979): In-Flight Acoustic Comparison of the 540 and $\mathrm{k} 747$ Main Rotors for the AH-1S Helicopter. Appendix, U.S. Army Aviation Engineering Flight Activity Report 77-38, Edwards AFB, Calif.

40. Shockey, G. A.; Williamson, J. W.; and Cos, C. R. (1976): Hel1copter AAerodynamics and Structural Loads Survey. Presented at the 32nd Annual National V/STOL Forum of the American Helicopter Society, Washington, D.C.

41. Sakowski, P.; and Charles, B. (1976): Noise Measurement Test Results for AH-1G Operational Loads Survey. vols. I and II. Report 299-099-831, Bell Helicopter Co.

42. George, A. R.; and Chang, S. B. (1983): Noise due to Transonic Blade-Vortex Interactions. Paper A-83-39-50-D000, 39th Annual National Forum of the American Helicopter Society.

43. Hubbard, J. E.; and Harris, W. L. (1979): An Investigation of Model Helicopter Rotor Blade Slap at Low Tip Speeds. AIAA Paper 79-0613, Seattle, Wash.

44. Leighton, K.; and Harris, W. L. (1983): A Parametric Study of Blade Vortex Interaction Noise for Two-, Three0, and Four-Bladed Mode1 Rotors at Moderate Tip Speeds: Theory and Experiment. Paper A-83-39-50-D000, 39th Annual National Forum of the American Helicopter Society.

45. Leverton, J. W.; and Taylor, F. W. (1966): Helicopter Blade Slap. J. Sound Vibration, vol. 4, no. 3, pp. 345-357. 


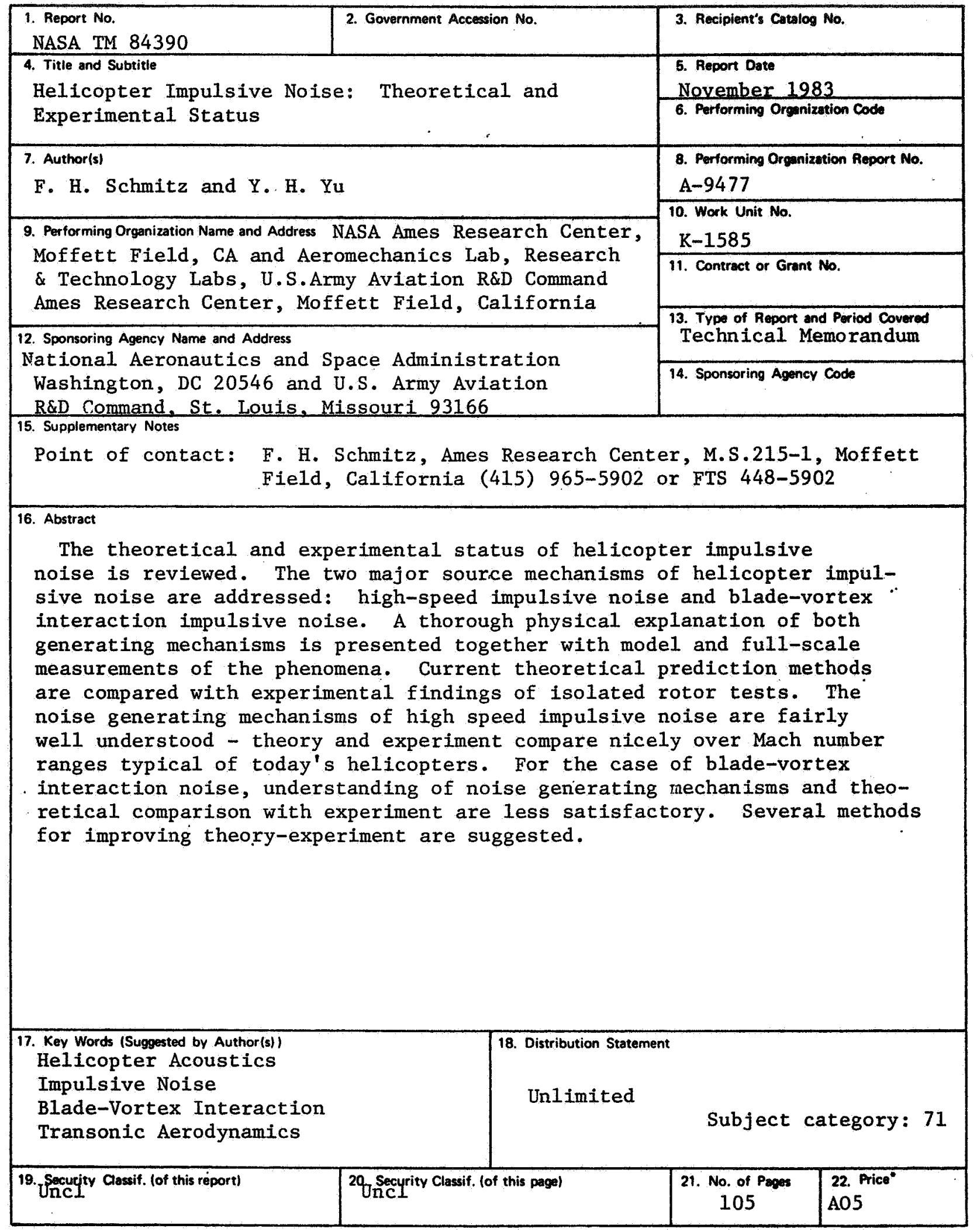

"For sale by the National Technical Information Service, Springfield, Virginia 22161 